\title{
Oxygen and Base-free Oxidative Heck Reactions of Arylboronic Acids with Olefins
}

\author{
Jiwu Ruan, Xinming Li, Ourida Saidi, and Jianliang Xiao* \\ Department of Chemistry, Liverpool Centre for Materials and Catalysis, \\ University of Liverpool, Liverpool L69 7ZD, U.K. \\ E-mail: j.xiao@liv.ac.uk
}

\section{Supporting Information}

\section{Table of contents}

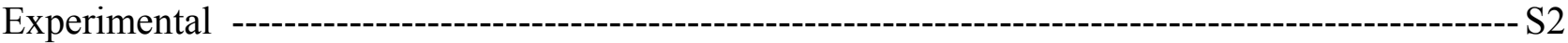

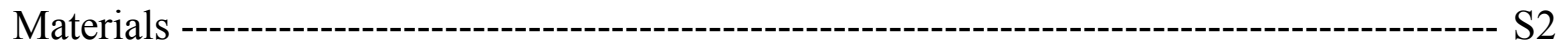

Table S1: Optimization of reaction conditions for the coupling of

4-methoxy-phenylboronic acid (1j) with $n$-butyl vinyl ether (2a) ---------------------- S2

General procedures for the oxidative Heck reaction of arylboronic acids (1) with $n$-butyl vinyl ether (2a) ---------------- S3

General procedures for the oxidative Heck reaction of arylboronic acids (1) with methyl acrylate (2f)----------------------------------------------------------------------- S3

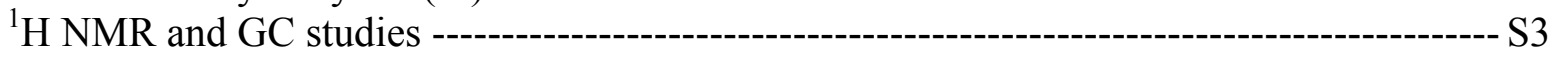

${ }^{1} \mathrm{H}$ NMR, ${ }^{13} \mathrm{C}$ NMR, IR and HRMS data of compounds 3a-z, 4a-b and 5a-u ------------------- S4

${ }^{1} \mathrm{H}$ NMR spectrum of the crude reaction solution resulting from the coupling of phenylboronic acid (1a) with methyl acrylate $(\mathbf{2 f})$ in $d_{6}$-acetone-----------------------------S16 phenylboronic acid (1a) with $n$-butyl vinyl ether (2a) in $d_{6}$-acetone ----------------------S16

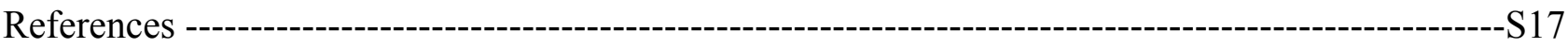

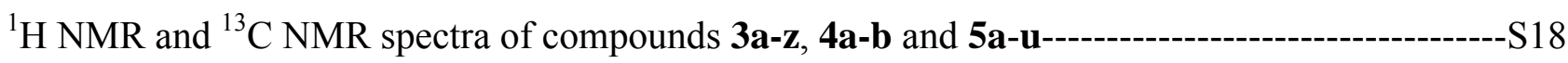




\section{Experimental}

Materials: All the reactions were carried out under a nitrogen atmosphere with dried solvents unless otherwise indicated. Silica gel plates $\left(\mathrm{GF}_{254}\right)$ were used for TLC and silica gel (230-400 mesh) was used for flash column chromatography. The following chemicals were purchased from Lancaster or Aldrich and used as received: all the arylboronic acids, $n$-butyl vinyl ether, 1-ethoxyprop-1-ene, 1-ethoxybut-1ene, 3-ethoxyprop-1-ene, allyl acetate, methyl acrylate, acrylonitrile, 1,3bis(diphenylphosphino)propane (dppp), 2,2'-bis(diphenylphosphino)-1,1'-binaphthalene (BINAP), 1,1'bis(diphenylphosphino)ferrocene (dppf), 4,5-bis(diphenylphosphino)-9,9-dimethylxanthene (Xantphos), triphenylphosphine $\left(\mathrm{PPh}_{3}\right), 2$,2'-bipyridine, trifluoroacetic acid (TFA), triflic acid (TfOH), $p$ toluenesulfonic acid (TsOH), acetic acid (HOAc), benzoic acid, $\mathrm{Pd}(\mathrm{OAc})_{2}, \mathrm{Pd}_{2}(\mathrm{dba})_{3}$, and methyl propionate. The ${ }^{1} \mathrm{H}$ and ${ }^{13} \mathrm{C}$ NMR spectra were recorded on a Bruker DRX-400 spectrometer with TMS as the internal standard, and infrared spectra on a JASCO FT/IR-4100 spectrometer. The mass spectra were obtained by electron impact (EI) or chemical ionization (CI). Gas chromatographic analysis was carried out on a Varian 3800 GC equipped with a CP WAX 52 CB column $(30 \mathrm{~m} \times 0.53 \mathrm{~mm})$ and an FID detector.

Table S1. Optimization of reaction conditions for the coupling of 4-methoxy-phenylboronic acid (1j) with $n$-butyl vinyl ether $(\mathbf{2 a})^{a}$

\begin{tabular}{|c|c|c|c|c|}
\hline & $1 \mathrm{j}$ & $2 a$ & & 3j \\
\hline entry & catalyst & ligand & solvent & yield $(\%)^{b}$ \\
\hline 1 & $\mathrm{Pd}_{2}(\mathrm{dba})_{3}$ & dppp & acetone & 73 \\
\hline 2 & $\mathrm{Pd}(\mathrm{OAc})_{2}$ & dppp & acetone & 89 \\
\hline 3 & $\mathrm{Pd}(\mathrm{OAc})_{2}$ & dppp & isopropanol & 27 \\
\hline 4 & $\mathrm{Pd}(\mathrm{OAc})_{2}$ & dppp & DMF & 67 \\
\hline 5 & $\mathrm{Pd}(\mathrm{OAc})_{2}$ & dppp & dioxane & 21 \\
\hline 6 & $\mathrm{Pd}(\mathrm{OAc})_{2}$ & none & acetone & $<5$ \\
\hline $7^{c}$ & $\mathrm{Pd}(\mathrm{OAc})_{2}$ & dppp & acetone & 71 \\
\hline 8 & $\mathrm{Pd}(\mathrm{OAc})_{2}$ & BINAP & acetone & 25 \\
\hline 9 & $\mathrm{Pd}(\mathrm{OAc})_{2}$ & dppf & acetone & 26 \\
\hline 10 & $\mathrm{Pd}(\mathrm{OAc})_{2}$ & Xantphos & acetone & 70 \\
\hline 11 & $\mathrm{Pd}(\mathrm{OAc})_{2}$ & $\mathrm{PPh}_{3}, 6 \mathrm{~mol} \%$ & acetone & $<5$ \\
\hline 12 & $\mathrm{Pd}(\mathrm{OAc})_{2}$ & 2,2'-bipyridine & acetone & $<5$ \\
\hline
\end{tabular}

\footnotetext{
${ }^{a}$ All reactions were carried out with $\mathbf{1 j}$ (1.0 mmol), 2a (2.0 equiv), [Pd] (2 mol\%), and ligand (3 mol\%) in $3 \mathrm{~mL}$ solvent at $70{ }^{\circ} \mathrm{C}$ for $15 \mathrm{~h}$. This was followed by further reaction with aqueous $\mathrm{HCl}$ and then workup. For details, please see the procedures immediately below. Due to the acidity of boronic acids, some $3 \mathbf{j}$ was formed before the $\mathrm{HCl}$ was added. ${ }^{b}$ Isolated yields. ${ }^{c}$ Reaction under $\mathrm{O}_{2}$.
} 
General procedures for the oxidative Heck reaction of arylboronic acids (1) with $n$-butyl vinyl ether (2a): An oven-dried carousel reaction tube containing a stirrer bar was charged with an arylboronic acid $1(1.0 \mathrm{mmol}), \mathrm{Pd}(\mathrm{OAc})_{2}(0.02 \mathrm{mmol})$, and dppp $(0.03 \mathrm{mmol})$. After degassing three times with nitrogen, $n$-butyl vinyl ether $2 \mathbf{a}(2.0 \mathrm{mmol})$ and freshly distilled acetone $(3 \mathrm{~mL})$ were injected sequentially. The reaction mixture was vigorously stirred at $70^{\circ} \mathrm{C}$ for $15 \mathrm{~h}$. After cooling down to room temperature, $3 \mathrm{~mL}$ aqueous $\mathrm{HCl}(3 \mathrm{M})$ was added and the mixture was stirred for $1 \mathrm{~h}$ at room temperature. $\mathrm{CH}_{2} \mathrm{Cl}_{2}(15 \mathrm{~mL})$ was then added, and the aqueous phase was extracted with $\mathrm{CH}_{2} \mathrm{Cl}_{2}(3 \times 10$ $\mathrm{mL}$ ). The combined organic phase was washed with $15 \mathrm{~mL}$ brine, dried over $\mathrm{MgSO}_{4}$, and concentrated in vacuo. The crude product was purified by flash chromatography on silica gel using a mixture of ethyl acetate and hexane (10/90 to 40/60) as eluant.

The optimization of the coupling of 4-methoxy-phenylboronic acid (1j) with $n$-butyl vinyl ether (2a) followed the same procedure; the results are shown in Table S1.

General procedures for the oxidative Heck reaction of arylboronic acids (1) with methyl acrylate (2f): An oven-dried carousel reaction tube containing a stirrer bar was charged with arylboronic acid $\mathbf{1}$ $(1.0 \mathrm{mmol}), \mathrm{Pd}(\mathrm{OAc})_{2}(0.02 \mathrm{mmol})$, and dppp $(0.03 \mathrm{mmol})$. After degassing three times with nitrogen, methyl acrylate $2 \mathrm{f}(2.0 \mathrm{mmol})$, freshly distilled acetone $(3 \mathrm{~mL})$, and TFA $(0.3 \mathrm{mmol})$ were injected sequentially. The reaction mixture was vigorously stirred at $70{ }^{\circ} \mathrm{C}$ for $20 \mathrm{~h}$. After cooling down to room temperature, the solvent was removed under vacuo. The crude product was purified by flash chromatography on silica gel using a mixture of ethyl acetate and hexane (10/90 to 50/50) as eluant.

The isolated yields of the coupling reactions reported in this study are given in Tables 1 and 2, and in equations 1 and 3.

${ }^{1} \mathbf{H}$ NMR and GC studies: These experiments were aimed to investigate whether the olefin substrate was reduced in the coupling reaction. An oven-dried carousel reaction tube containing a stirrer bar was charged with arylboronic acid 1a $(0.5 \mathrm{mmol}), \mathrm{Pd}(\mathrm{OAc})_{2}(0.01 \mathrm{mmol})$, and dppp $(0.015 \mathrm{mmol})$. After degassing three times with nitrogen, olefin $2 \mathbf{a}$ or $2 \mathbf{f}(0.75 \mathrm{mmol})$ and $d_{6}$-acetone $(2 \mathrm{~mL})$ were injected sequentially. In the case of $\mathbf{2 f}$, TFA $(0.15 \mathrm{mmol})$ was then added. The reaction mixture was vigorously stirred at $70{ }^{\circ} \mathrm{C}$ for a period of time given in Tables 1 and 2 of the text. After cooling down to room temperature, the crude reaction solution was analyzed by ${ }^{1} \mathrm{H}$ NMR, and by GC after passing through a pad of celite. No difference in the ${ }^{1} \mathrm{H}$ NMR spectra of the resulting products were observed when 1a was replaced with $\mathrm{C}_{6} \mathrm{H}_{5}-\mathrm{B}(\mathrm{OD})_{2}$. 
${ }^{1} \mathrm{H}$ NMR, ${ }^{13} \mathrm{C}$ NMR, IR, and HRMS data of compounds 3a-z, 4a-b and 5a-u:

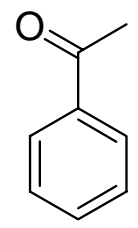

$3 a$

Acetophenone (3a). ${ }^{1}{ }^{1} \mathrm{H} \mathrm{NMR}\left(400 \mathrm{MHz}, \mathrm{CDCl}_{3}\right) \delta$ 7.97-7.94 (m, 2H), 7.58-7.54 (m, $\left.1 \mathrm{H}\right)$, 7.48-7.44 $(\mathrm{m}, 2 \mathrm{H}), 2.60(\mathrm{~s}, 3 \mathrm{H}) ;{ }^{13} \mathrm{C} \mathrm{NMR}\left(100 \mathrm{MHz}, \mathrm{CDCl}_{3}\right) \delta 198.5,137.6,133.5,129.0,128.7,26.9$; IR (neat, $\left.\mathrm{cm}^{-1}\right)$ : 1681.6; HRMS calcd for $\mathrm{C}_{8} \mathrm{H}_{8} \mathrm{O}$ : 120.0575; Found: 120.0576 .<smiles>CC(=O)c1ccccc1C</smiles>

3b

2'-Methylacetophenone (3b). ${ }^{2}{ }^{1} \mathrm{H}$ NMR $\left(400 \mathrm{MHz}, \mathrm{CDCl}_{3}\right) \delta$ 7.69-7.67 (m, 1H), 7.37-7.34 (m, $\left.1 \mathrm{H}\right)$, 7.27-7.22 (m, 2H), $2.57(\mathrm{~s}, 3 \mathrm{H}), 2.53(\mathrm{~s}, 3 \mathrm{H}) ;{ }^{13} \mathrm{C} \mathrm{NMR}\left(100 \mathrm{MHz}, \mathrm{CDCl}_{3}\right) \delta 202.1,138.8,138.2,132.4$, 131.9, 129.7, 126.1, 29.9, 21.9; IR (neat, $\mathrm{cm}^{-1}$ ): 1679; HRMS calcd for $\mathrm{C}_{9} \mathrm{H}_{10} \mathrm{O}$ : 134.0732; Found: 134.0731 .<smiles>COc1ccccc1C(C)=O</smiles>

3c

2'-Methoxyacetophenone (3c). ${ }^{1}{ }^{1} \mathrm{H}$ NMR $\left(400 \mathrm{MHz}, \mathrm{CDCl}_{3}\right) \delta$ 7.74-7.71 (m, 1H), 7.48-7.43 (m, $\left.1 \mathrm{H}\right)$, 7.01-6.95 (m, 2H), $3.91(\mathrm{~s}, 3 \mathrm{H}), 2.61(\mathrm{~s}, 3 \mathrm{H}) ;{ }^{13} \mathrm{C} \mathrm{NMR}\left(100 \mathrm{MHz}, \mathrm{CDCl}_{3}\right) \delta 200.2,159.3,134.0,130.7$, 128.8, 121.0, 112.0, 55.9, 32.2; IR (neat, $\mathrm{cm}^{-1}$ ): 1670; HRMS calcd for $\mathrm{C}_{9} \mathrm{H}_{10} \mathrm{O}_{2}$ : 150.0681; Found: 150.0683 .<smiles>CC(=O)c1ccccc1Cl</smiles>

3d

2'-Chloroacetophenone (3d). ${ }^{3}{ }^{1} \mathrm{H} \mathrm{NMR}\left(400 \mathrm{MHz}, \mathrm{CDCl}_{3}\right) \delta$ 7.56-7.53 (m, 1H), 7.41-7.30 (m, $\left.3 \mathrm{H}\right)$, $2.64(\mathrm{~s}, 3 \mathrm{H}) ;{ }^{13} \mathrm{C}$ NMR $\left(100 \mathrm{MHz}, \mathrm{CDCl}_{3}\right) \delta 200.8,139.6,132.3,131.7,131.0,129.8,127.3,31.1 ; \mathrm{IR}$ (neat, $\mathrm{cm}^{-1}$ ): 1699; HRMS calcd for $\mathrm{C}_{8} \mathrm{H}_{7} \mathrm{ClO}$ : 154.0185 and 156.0156; Found: 154.0183 and 156.0160. 


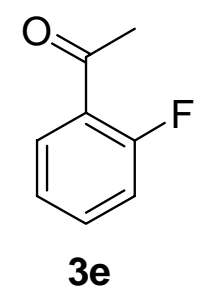

2'-Fluoroacetophenone (3e). ${ }^{1}{ }^{1} \mathrm{H}$ NMR $\left(400 \mathrm{MHz}, \mathrm{CDCl}_{3}\right) \delta$ 7.89-7.85 (m, 1H), 7.53-7.49 (m, 1H), 7.24-7.20 (m, 1 H), 7.16-7.11 (m, 1H), $2.64(\mathrm{~d}, J=5 \mathrm{~Hz}, 3 \mathrm{H}) ;{ }^{13} \mathrm{C}$ NMR $\left(100 \mathrm{MHz}, \mathrm{CDCl}_{3}\right) \delta 196.3$, $162.7\left(\mathrm{~d}, J_{\mathrm{CF}}=253 \mathrm{~Hz}\right), 135.0\left(\mathrm{~d}, J_{\mathrm{CF}}=9 \mathrm{~Hz}\right), 131.0\left(\mathrm{~d}, J_{\mathrm{CF}}=3 \mathrm{~Hz}\right), 126.2\left(\mathrm{~d}, J_{\mathrm{CF}}=13 \mathrm{~Hz}\right), 124.7(\mathrm{~d}$, $\left.J_{\mathrm{CF}}=4 \mathrm{~Hz}\right), 116.6\left(\mathrm{~d}, J_{\mathrm{CF}}=24 \mathrm{~Hz}\right), 31.7\left(\mathrm{~d}, J_{\mathrm{CF}}=8 \mathrm{~Hz}\right)$; IR (neat, $\left.\mathrm{cm}^{-1}\right): 1687$; HRMS calcd for $\mathrm{C}_{8} \mathrm{H}_{7} \mathrm{FO}: 138.0481$; Found: 138.0483 .

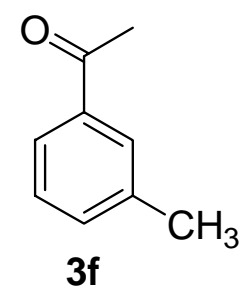

3'-Methylacetophenone (3f) ${ }^{1}{ }^{1} \mathrm{H}$ NMR $\left(400 \mathrm{MHz}, \mathrm{CDCl}_{3}\right) \delta$ 7.77-7.74 (m, 2H), 7.37-7.34 (m, 2H), 2.59 (s, 3H), $2.41(\mathrm{~s}, 3 \mathrm{H}) ;{ }^{13} \mathrm{C}$ NMR $\left(100 \mathrm{MHz}, \mathrm{CDCl}_{3}\right) \delta 198.7,138.8,137.7,134.2,129.2,128.8$, 126.0, 27.0, 21.7; IR (neat, $\mathrm{cm}^{-1}$ ): 1679; HRMS calcd for $\mathrm{C}_{9} \mathrm{H}_{10} \mathrm{O}$ : 134.0732; Found: 134.0734.

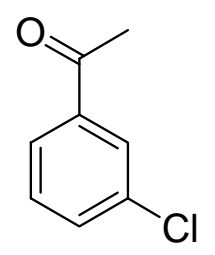

$3 g$

3'-Chloroacetophenone (3g). ${ }^{4}{ }^{1} \mathrm{H}$ NMR $\left(400 \mathrm{MHz}, \mathrm{CDCl}_{3}\right) \delta$ 7.93-7.92 (m, 1H), 7.84-7.82 (m, 1H), 7.55-7.52 (m, 1H), 7.43-7.39 (m, 1H), $2.59(\mathrm{~s}, 3 \mathrm{H}) ;{ }^{13} \mathrm{C}$ NMR (100 MHz, $\left.\mathrm{CDCl}_{3}\right) \delta$ 197.0, 139.1, 135.4, 133.4, 130.3, 128.8, 126.8, 27.0; IR (neat, $\mathrm{cm}^{-1}$ ): 1687; HRMS calcd for $\mathrm{C}_{8} \mathrm{H}_{7} \mathrm{ClO}$ : 154.0185 and 156.0156; Found: 154.0184 and 156.0158.

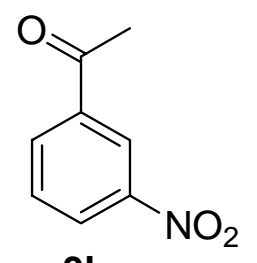

$3 \mathrm{~h}$

3'-Nitroacetophenone (3h). ${ }^{4} \mathrm{H}$ NMR (400 MHz, $\left.\mathrm{CDCl}_{3}\right) \delta 8.77$ (d, $\left.J=2.0 \mathrm{~Hz}, 1 \mathrm{H}\right), 8.44-8.41(\mathrm{~m}, 1 \mathrm{H})$, 8.30-8.28 (m, 1H), 7.71-7.67 (m, 1H), $2.69(\mathrm{~s}, 3 \mathrm{H}) ;{ }^{13} \mathrm{C}$ NMR $\left(100 \mathrm{MHz}, \mathrm{CDCl}_{3}\right) \delta 196.0,148.9,138.7$, 134.1, 130.3, 127.8, 123.6, 27.1; IR (neat, $\mathrm{cm}^{-1}$ ): 1689, 1523, 1344; HRMS calcd for $\mathrm{C}_{8} \mathrm{H}_{7} \mathrm{NO}_{3}$ : 165.0426; Found: 165.0428. 


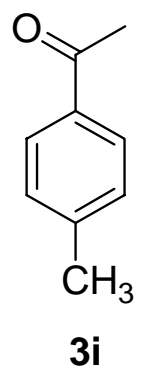

4'-Methylacetophenone (3i). ${ }^{1} \mathrm{H}$ NMR (400 MHz, $\left.\mathrm{CDCl}_{3}\right) \delta 7.85$ (d, $\left.J=8 \mathrm{~Hz}, 2 \mathrm{H}\right), 7.25(\mathrm{~d}, J=8 \mathrm{~Hz}$, $2 \mathrm{H}), 2.57(\mathrm{~s}, 3 \mathrm{H}), 2.41(\mathrm{~s}, 3 \mathrm{H}) ;{ }^{13} \mathrm{C} \mathrm{NMR}\left(100 \mathrm{MHz}, \mathrm{CDCl}_{3}\right) \delta 198.2,144.2,135.2,129.6,128.8,26.9$, 22.0; IR (neat, $\mathrm{cm}^{-1}$ ): 1677; HRMS calcd for $\mathrm{C}_{9} \mathrm{H}_{10} \mathrm{O}$ : 134.0732; Found: 134.0735.

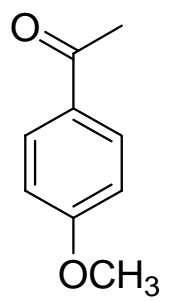

3j

4'-Methoxyacetophenone (3j). ${ }^{1}{ }^{1} \mathrm{H}$ NMR $\left(400 \mathrm{MHz}, \mathrm{CDCl}_{3}\right) \delta 7.93(\mathrm{~d}, J=8.8 \mathrm{~Hz}, 2 \mathrm{H}), 6.92(\mathrm{~d}, J=$ $8.8 \mathrm{~Hz}, 2 \mathrm{H}), 3.86(\mathrm{~s}, 3 \mathrm{H}), 2.54(\mathrm{~s}, 3 \mathrm{H}) ;{ }^{13} \mathrm{C}$ NMR $\left(100 \mathrm{MHz}, \mathrm{CDCl}_{3}\right) \delta 197.1,163.9,131.0,130.8,114.1$, 55.8, 26.7; IR (neat, $\left.\mathrm{cm}^{-1}\right)$ : 1664; HRMS Calcd for $\mathrm{C}_{9} \mathrm{H}_{11} \mathrm{O}_{2}(\mathrm{M}+\mathrm{H})^{+}$: 151.0759; Found: 151.0762.

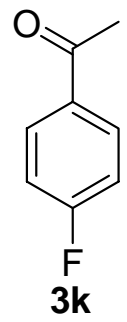

4'-Fluoroacetophenone (3k). ${ }^{1}{ }^{1} \mathrm{H}$ NMR $\left(400 \mathrm{MHz}, \mathrm{CDCl}_{3}\right) \delta$ 8.00-7.96 (m, 2H), 7.15-7.11 (m, 2H), $2.58(\mathrm{~s}, 3 \mathrm{H}) ;{ }^{13} \mathrm{C}$ NMR $\left(100 \mathrm{MHz}, \mathrm{CDCl}_{3}\right) \delta 196.8,166.2\left(\mathrm{~d}, J_{\mathrm{CF}}=254 \mathrm{~Hz}\right), 134.0\left(\mathrm{~d}, J_{\mathrm{CF}}=3 \mathrm{~Hz}\right)$, $131.3\left(\mathrm{~d}, J_{\mathrm{CF}}=9 \mathrm{~Hz}\right), 116.0\left(\mathrm{~d}, J_{\mathrm{CF}}=22 \mathrm{~Hz}\right), 26.9$; IR (neat, $\left.\mathrm{cm}^{-1}\right): 1681$; HRMS calcd for $\mathrm{C}_{8} \mathrm{H}_{7} \mathrm{FO}$ : 138.0481; Found: 138.0480.

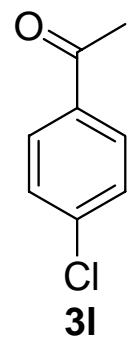

4'-Chloroacetophenone (3l). ${ }^{2} \mathrm{H}$ NMR $\left(400 \mathrm{MHz}, \mathrm{CDCl}_{3}\right) \delta 7.88(\mathrm{~d}, J=8.8 \mathrm{~Hz}, 2 \mathrm{H}), 7.42(\mathrm{~d}, J=8.8$ $\mathrm{Hz}, 2 \mathrm{H}), 2.58(\mathrm{~s}, 3 \mathrm{H}) ;{ }^{13} \mathrm{C}$ NMR $\left(100 \mathrm{MHz}, \mathrm{CDCl}_{3}\right) \delta 197.1,139.9,135.9,130.1,129.3,26.9$; IR (neat, $\mathrm{cm}^{-1}$ ): 1681; HRMS calcd for $\mathrm{C}_{8} \mathrm{H}_{7} \mathrm{ClO}: 154.0185$ and 156.0156; Found: 154.0187 and 156.0160 . 


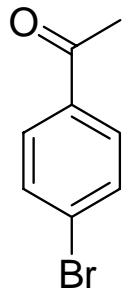

$3 m$

4'-Bromoacetophenone (3m). ${ }^{2}{ }^{1} \mathrm{H}$ NMR $\left(400 \mathrm{MHz}, \mathrm{CDCl}_{3}\right) \delta 7.81$ (d, $\left.J=8.8 \mathrm{~Hz}, 2 \mathrm{H}\right), 7.60$ (d, $J=8.8$ $\mathrm{Hz}, 2 \mathrm{H}), 2.58(\mathrm{~s}, 3 \mathrm{H}) ;{ }^{13} \mathrm{C} \mathrm{NMR}\left(100 \mathrm{MHz}, \mathrm{CDCl}_{3}\right) \delta 197.3,136.3,132.3,130.2,128.7,26.9$; IR (neat, $\mathrm{cm}^{-1}$ ): 1670; HRMS calcd for $\mathrm{C}_{8} \mathrm{H}_{7} \mathrm{BrO}$ : 197.9680 and 199.9660; Found: 197.9677 and 199.9655.<smiles>CC(=O)c1ccc([N+](=O)[O-])cc1</smiles>

$3 n$

4'-Nitroacetophenone (3n). ${ }^{2}{ }^{1} \mathrm{H}$ NMR (400 MHz, $\left.\mathrm{CDCl}_{3}\right) \delta 8.33-8.30$ (m, 2H), 8.13-8.10 (m, 2H), 2.68 (s, 3H); ${ }^{13} \mathrm{C}$ NMR $\left(100 \mathrm{MHz}, \mathrm{CDCl}_{3}\right) \delta 196.6,150.8,141.8,129.6,124.2,27.3$; IR (neat, $\left.\mathrm{cm}^{-1}\right): 1689$, 1521, 1342; HRMS calcd for $\mathrm{C}_{8} \mathrm{H}_{7} \mathrm{NO}_{3}$ : 165.0426; Found: 165.0426 .

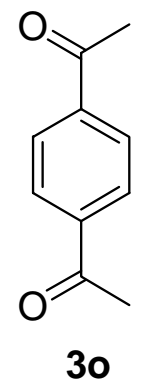

1,4-Diacetylbenzene (3o). ${ }^{1}{ }^{1} \mathrm{H}$ NMR $\left(400 \mathrm{MHz}, \mathrm{CDCl}_{3}\right) \delta 8.03$ (s, 4H), 2.65 (s, 6H); ${ }^{13} \mathrm{C}$ NMR $(100$ $\left.\mathrm{MHz}, \mathrm{CDCl}_{3}\right) \delta 197.8,140.6,128.9,27.2$; IR $\left(\right.$ neat, $\mathrm{cm}^{-1}$ ): 1675; HRMS calcd for $\mathrm{C}_{10} \mathrm{H}_{10} \mathrm{O}_{2}: 162.0681$; Found: 162.0684 .

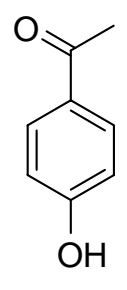

$3 p$

4'-Hydroxyacetophenone (3p). ${ }^{6}{ }^{1} \mathrm{H}$ NMR $\left(400 \mathrm{MHz}, \mathrm{CDCl}_{3}\right) \delta 7.92(\mathrm{~d}, J=8.4 \mathrm{~Hz}, 2 \mathrm{H}), 7.34(\mathrm{~s}, 1 \mathrm{H})$, $6.94(\mathrm{~d}, J=8.4 \mathrm{~Hz}, 2 \mathrm{H}), 2.59(\mathrm{~s}, 3 \mathrm{H}) ;{ }^{13} \mathrm{C} \mathrm{NMR}\left(100 \mathrm{MHz}, \mathrm{CDCl}_{3}\right) \delta 198.8,161.5,131.6,130.2,115.9$, 26.8; IR (neat, $\mathrm{cm}^{-1}$ ): 3103, 1643; HRMS Calcd for $\mathrm{C}_{8} \mathrm{H}_{8} \mathrm{O}_{2}$ : 136.0524; Found: 136.0523. 


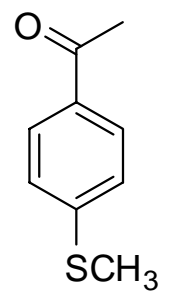

$3 q$

4'-Methylthioacetophenone (3q). ${ }^{7} \mathrm{H}$ NMR (400 MHz, $\left.\mathrm{CDCl}_{3}\right) \delta 7.86(\mathrm{~d}, J=8.4 \mathrm{~Hz}, 2 \mathrm{H}), 7.25(\mathrm{~d}, J=$ $8.4 \mathrm{~Hz}, 2 \mathrm{H}), 2.57$ (s, 3H), $2.52(\mathrm{~s}, 3 \mathrm{H}) ;{ }^{13} \mathrm{C} \mathrm{NMR}\left(100 \mathrm{MHz}, \mathrm{CDCl}_{3}\right) \delta$ 197.6, 146.3, 133.7, 129.1, 125.3, 26.8, 15.2; IR (neat, $\mathrm{cm}^{-1}$ ): 1666; HRMS Calcd for $\mathrm{C}_{9} \mathrm{H}_{10} \mathrm{OS}$ : 166.0452; Found: 166.0450.

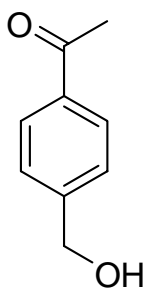

$3 r$

4'-Hydroxymethylacetophenone (3r). ${ }^{8}{ }^{1} \mathrm{H}$ NMR (400 MHz, $\left.\mathrm{CDCl}_{3}\right) \delta 7.90$ (d, J=8.4 Hz, 2H), 7.42 (d, $J=8.4 \mathrm{~Hz}, 2 \mathrm{H}), 4.74(\mathrm{~s}, 3 \mathrm{H}), 2.57(\mathrm{~s}, 3 \mathrm{H}) ;{ }^{13} \mathrm{C} \mathrm{NMR}\left(100 \mathrm{MHz}, \mathrm{CDCl}_{3}\right) \delta 198.8,146.03,135.5,129.0$, 127.0, 64.8, 27.1; IR (neat, $\mathrm{cm}^{-1}$ ): 3423, 1651; HRMS Calcd for $\mathrm{C}_{9} \mathrm{H}_{10} \mathrm{O}_{2}$ : 150.0681; Found: 150.0677.

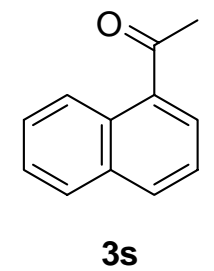

1'-Acetonaphthone (3s). ${ }^{1}{ }^{1} \mathrm{H}$ NMR $\left(400 \mathrm{MHz}, \mathrm{CDCl}_{3}\right) \delta$ 8.75-8.73 (m, 1H), 7.99-7.85 (m, 3H), 7.60$7.46(\mathrm{~m}, 3 \mathrm{H}), 2.73(\mathrm{~s}, 3 \mathrm{H}) ;{ }^{13} \mathrm{C} \mathrm{NMR}\left(100 \mathrm{MHz}, \mathrm{CDCl}_{3}\right) \delta 202.2,136.0,134.4,133.4,130.6,129.0$, 128.8, 128.4, 126.8, 126.4, 124.7, 30.4; IR (neat, $\mathrm{cm}^{-1}$ ): 1674; HRMS calcd for $\mathrm{C}_{12} \mathrm{H}_{10} \mathrm{O}: 170.0732$; Found: 170.0728 .

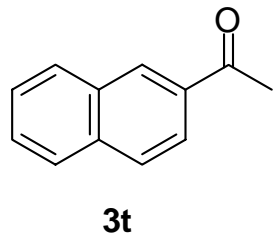

2'-Acetonaphthone (3t). ${ }^{9} \mathrm{H}$ NMR $\left(400 \mathrm{MHz}^{1} \mathrm{CDCl}_{3}\right) \delta 8.46(\mathrm{~s}, 1 \mathrm{H}), 8.05-8.02(\mathrm{~m}, 1 \mathrm{H}), 7.98-7.95$ (m, 1H), 7.90-7.86 (m, 2H), 7.62-7.53 (m, 2H), $2.72(\mathrm{~s}, 3 \mathrm{H}) ;{ }^{13} \mathrm{C}$ NMR $\left(100 \mathrm{MHz}, \mathrm{CDCl}_{3}\right) \delta 198.4,136.0$, 135.0, 133.0, 130.6, 130.0, 128.9, 128.8, 128.2, 127.2, 124.3, 27.0; IR (neat, $\mathrm{cm}^{-1}$ ): 1670; HRMS calcd for $\mathrm{C}_{12} \mathrm{H}_{10} \mathrm{O}$ : 170.0732 ; Found: 170.0731 . 


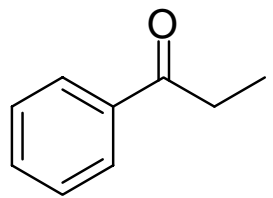

3u

Propiophenone (3u). ${ }^{2}{ }^{1} \mathrm{H}$ NMR (400 MHz, $\left.\mathrm{CDCl}_{3}\right) \delta$ 7.98-7.95 (m, 2H), 7.57-7.53 (m, 1H), 7.47-7.43 $(\mathrm{m}, 2 \mathrm{H}), 3.10(\mathrm{q}, J=7 \mathrm{~Hz}, 2 \mathrm{H}), 1.23(\mathrm{t}, J=7 \mathrm{~Hz}, 3 \mathrm{H}) ;{ }^{13} \mathrm{C} \mathrm{NMR}\left(100 \mathrm{MHz}, \mathrm{CDCl}_{3}\right) \delta 201.2,137.3$, 133.3, 129.0, 128.4, 32.2, 8.7; IR (neat, $\mathrm{cm}^{-1}$ ): 1685; HRMS calcd for $\mathrm{C}_{9} \mathrm{H}_{10} \mathrm{O}$ : 134.0732; Found: 134.0730 .<smiles>CCC(=O)c1ccc(OC)cc1</smiles>

1-(4-Methoxyphenyl)propan-1-one (3v). ${ }^{10}{ }^{1} \mathrm{H}$ NMR (400 MHz, $\left.\mathrm{CDCl}_{3}\right) \delta 7.96(\mathrm{~d}, J=7 \mathrm{~Hz}, 2 \mathrm{H}), 6.95$ $(\mathrm{d}, J=7 \mathrm{~Hz}, 2 \mathrm{H}), 3.88(\mathrm{~s}, 3 \mathrm{H}), 2.96(\mathrm{q}, J=7 \mathrm{~Hz}, 2 \mathrm{H}), 1.22(\mathrm{t}, J=7 \mathrm{~Hz}, 3 \mathrm{H}) ;{ }^{13} \mathrm{C}$ NMR $\left(100 \mathrm{MHz}, \mathrm{CDCl}_{3}\right)$ $\delta$ 199.9, 163.7, 130.6, 130.4, 114.1, 55.8, 31.8, 8.8; IR (neat, $\mathrm{cm}^{-1}$ ): 1676; HRMS calcd for $\mathrm{C}_{10} \mathrm{H}_{12} \mathrm{O}_{2}$ : 164.0837; Found: 164.0834.<smiles>CCC(=O)c1ccc(Br)cc1</smiles>

1-(4-Bromophenyl)propan-1-one (3w). ${ }^{1} \mathrm{H}$ NMR $\left(400 \mathrm{MHz}, \mathrm{CDCl}_{3}\right) \delta 7.83(\mathrm{~d}, J=7 \mathrm{~Hz}, 2 \mathrm{H}), 7.60(\mathrm{~d}$, $J=7 \mathrm{~Hz}, 2 \mathrm{H}), 2.97(\mathrm{q}, J=7 \mathrm{~Hz}, 2 \mathrm{H}), 1.22(\mathrm{t}, J=7 \mathrm{~Hz}, 3 \mathrm{H}) ;{ }^{13} \mathrm{C}$ NMR $\left(100 \mathrm{MHz}, \mathrm{CDCl}_{3}\right) \delta 200.1,136.0$, 132.3, 129.9, 128.4, 32.2, 8.5; IR (neat, $\mathrm{cm}^{-1}$ ): 1683; HRMS calcd for $\mathrm{C}_{9} \mathrm{H}_{9} \mathrm{BrO}$ : 211.9837 and 213.9816; Found: 211.9840 and 213.9822 .

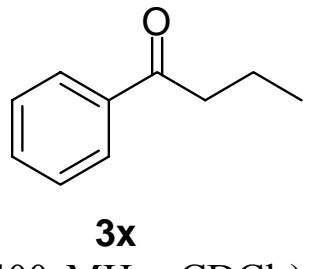

1-Phenylbutan-1-one (3x). ${ }^{11}{ }^{1} \mathrm{H}$ NMR $\left(400 \mathrm{MHz}, \mathrm{CDCl}_{3}\right) \delta$ 7.98-7.95 (m, 2H), 7.57-7.53 (m, 1H), 7.48-7.44 (m, 2H), $2.95(\mathrm{t}, J=7 \mathrm{~Hz}, 2 \mathrm{H}), 1.80-1.75(\mathrm{~m}, 2 \mathrm{H}), 1.01(\mathrm{t}, J=7 \mathrm{~Hz}, 3 \mathrm{H}) ;{ }^{13} \mathrm{C} \mathrm{NMR}(100 \mathrm{MHz}$, $\left.\mathrm{CDCl}_{3}\right) \delta 200.9,137.5,133.3,129.0,128.5,40.9,18.2,14.3$; IR (neat, $\mathrm{cm}^{-1}$ ): 1683; HRMS calcd for $\mathrm{C}_{10} \mathrm{H}_{12} \mathrm{O}$ : 148.0888; Found: 148.0886 . 


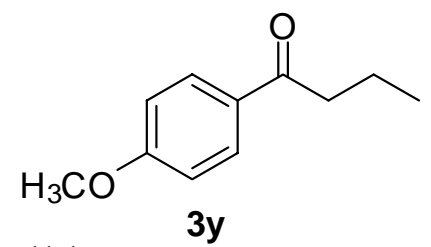

1-(4-Methoxyphenyl)butan-1-one (3y). ${ }^{11}{ }^{1} \mathrm{H}$ NMR $\left(400 \mathrm{MHz}, \mathrm{CDCl}_{3}\right) \delta 7.94(\mathrm{~d}, J=9 \mathrm{~Hz}, 2 \mathrm{H}), 6.92(\mathrm{~d}$, $J=9 \mathrm{~Hz}, 2 \mathrm{H}), 3.86(\mathrm{~s}, 3 \mathrm{H}), 2.89(\mathrm{t}, J=7 \mathrm{~Hz}, 2 \mathrm{H}), 1.78-1.73(\mathrm{~m}, 2 \mathrm{H}), 1.00(\mathrm{t}, J=7 \mathrm{~Hz}, 3 \mathrm{H}) ;{ }^{13} \mathrm{C} \mathrm{NMR}$ $\left(100 \mathrm{MHz}, \mathrm{CDCl}_{3}\right) \delta 199.5,163.7,130.6,130.6,114.1,55.8,40.6,18.4,14.3$; IR (neat, $\left.\mathrm{cm}^{-1}\right): 1674$; HRMS calcd for $\mathrm{C}_{11} \mathrm{H}_{14} \mathrm{O}_{2}$ : 178.0994; Found: 178.0996 .

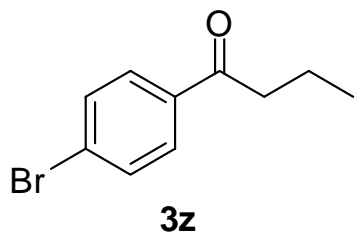

1-(4-Bromophenyl)butan-1-one (3z). ${ }^{12}{ }^{1} \mathrm{H}$ NMR $\left(400 \mathrm{MHz}, \mathrm{CDCl}_{3}\right) \delta 7.82(\mathrm{~d}, J=9 \mathrm{~Hz}, 2 \mathrm{H}), 7.60(\mathrm{~d}, J$ $=9 \mathrm{~Hz}, 2 \mathrm{H}), 2.91(\mathrm{t}, J=7 \mathrm{~Hz}, 2 \mathrm{H}), 1.79-1.74(\mathrm{~m}, 2 \mathrm{H}), 1.00(\mathrm{t}, J=7 \mathrm{~Hz}, 3 \mathrm{H}) ;{ }^{13} \mathrm{C}$ NMR $(100 \mathrm{MHz}$, $\left.\mathrm{CDCl}_{3}\right) \delta 199.7,136.2,132.3,130.0,128.4,40.9,18.1,14.2$; IR (neat, $\mathrm{cm}^{-1}$ ): 1691; HRMS calcd for $\mathrm{C}_{10} \mathrm{H}_{11} \mathrm{BrO}$ : 225.9993 and 227.9973; Found: 225.9990 and 227.9970.

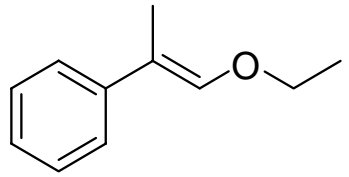

$4 a^{\prime}$

(E)-(1-ethoxyprop-1-en-2-yl)benzene (4a'). ${ }^{13} \mathrm{H}$ NMR $\left(400 \mathrm{MHz}, \mathrm{CDCl}_{3}\right) \delta$ 7.32-7.19 (m, 4H), 7.19$7.17(\mathrm{~m}, 1 \mathrm{H}), 6.48(\mathrm{~s}, 1 \mathrm{H}), 3.92(\mathrm{q}, J=7 \mathrm{~Hz}, 2 \mathrm{H}), 2.00(\mathrm{~s}, 3 \mathrm{H}), 1.32(\mathrm{t}, J=7 \mathrm{~Hz}, 3 \mathrm{H}) ;{ }^{13} \mathrm{C}$ NMR $(100$ $\left.\mathrm{MHz}, \mathrm{CDCl}_{3}\right) \delta 144.2,141.3,128.7,126.2,125.4,114.8,68.5,15.9,13.1 ; \mathrm{IR}\left(\right.$ neat, $\left.\mathrm{cm}^{-1}\right): 1267 ; \mathrm{HRMS}$ calcd for $\mathrm{C}_{11} \mathrm{H}_{14} \mathrm{O}$ : 162.1045 ; Found: 162.1044 .<smiles>C=C(COC(C)=O)c1ccccc1</smiles>

$4 b$

2-Phenylallyl acetate (4b). ${ }^{14}{ }^{1} \mathrm{H} \mathrm{NMR}\left(400 \mathrm{MHz}, \mathrm{CDCl}_{3}\right) \delta$ 7.45-7.42 (m, 2H), 7.37-7.31 (m, 3H), 5.56 $(\mathrm{s}, 1 \mathrm{H}), 5.37(\mathrm{~s}, 1 \mathrm{H}), 4.98(\mathrm{~s}, 2 \mathrm{H}), 2.08(\mathrm{~s}, 3 \mathrm{H}) ;{ }^{13} \mathrm{C} \mathrm{NMR}\left(100 \mathrm{MHz}, \mathrm{CDCl}_{3}\right) \delta 171.2,142.9,138.4$, 128.9, 128.5, 126.4, 115.7, 66.2, 21.4; IR (neat, $\mathrm{cm}^{-1}$ ): 1741; HRMS calcd for $\mathrm{C}_{11} \mathrm{H}_{12} \mathrm{O}_{2}$ : 176.0837; Found: 176.0834 .

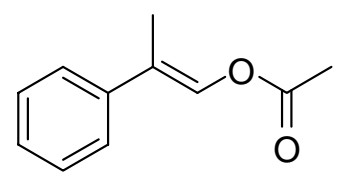

4b'

(E)-2-Phenylprop-1-enyl acetate (4b'). ${ }^{14}{ }^{1} \mathrm{H}$ NMR $\left(400 \mathrm{MHz}, \mathrm{CDCl}_{3}\right) \delta 7.51$ (s, 1H), 7.40-7.37 (m, $2 \mathrm{H}), 7.35-7.31(\mathrm{~m}, 2 \mathrm{H}), 7.28-7.26(\mathrm{~m}, 1 \mathrm{H}), 2.22(\mathrm{~s}, 3 \mathrm{H}), 2.10(\mathrm{~s}, 3 \mathrm{H}) ;{ }^{13} \mathrm{C}$ NMR $\left(100 \mathrm{MHz}, \mathrm{CDCl}_{3}\right) \delta$ $168.5,139.5,133.0,128.9,127.7,126.2,122.0,21.3,14.0$; IR $\left(\right.$ neat, $\mathrm{cm}^{-1}$ ): 1751; HRMS calcd for $\mathrm{C}_{11} \mathrm{H}_{12} \mathrm{O}_{2}:$ : 176.0837; Found: 176.0836. 
<smiles>COC(=O)/C=C/c1ccccc1</smiles>

$5 a$

Methyl trans-cinnamate (5a). ${ }^{15}{ }^{1} \mathrm{H}$ NMR $\left(400 \mathrm{MHz}, \mathrm{CDCl}_{3}\right) \delta 7.69(\mathrm{~d}, J=16 \mathrm{~Hz}, 1 \mathrm{H}), 7.53-7.51(\mathrm{~m}, 2$ $\mathrm{H}), 7.39-7.37(\mathrm{~m}, 3 \mathrm{H}), 6.44(\mathrm{~d}, J=16 \mathrm{~Hz}, 1 \mathrm{H}), 3.81(\mathrm{~s}, 3 \mathrm{H}) ;{ }^{13} \mathrm{C} \mathrm{NMR}\left(100 \mathrm{MHz}, \mathrm{CDCl}_{3}\right) \delta 167.8$, 145.3, 134.9, 130.7, 129.3, 128.5, 118.3, 52.0; IR (neat, $\mathrm{cm}^{-1}$ ): 1712; HRMS calcd for $\mathrm{C}_{10} \mathrm{H}_{10} \mathrm{O}_{2}$ : 162.0681; Found: 162.0684.

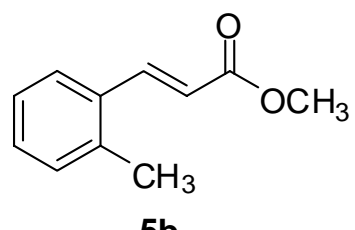

(E)-Methyl 3-o-tolylacrylate (5b). $\left.{ }^{15}{ }^{1} \mathrm{H} \mathrm{NMR} \mathrm{(400} \mathrm{MHz,} \mathrm{CDCl}_{3}\right) \delta 7.98$ (d, $\left.J=16 \mathrm{~Hz}, 1 \mathrm{H}\right), 7.53$ (d, $J$ $=8 \mathrm{~Hz}, 1 \mathrm{H}), 7.26-7.18(\mathrm{~m}, 3 \mathrm{H}), 6.35(\mathrm{~d}, J=16 \mathrm{~Hz}, 1 \mathrm{H}), 3.80(\mathrm{~s}, 3 \mathrm{H}), 2.43(\mathrm{~s}, 3 \mathrm{H}) ;{ }^{13} \mathrm{C}$ NMR $(100$ $\mathrm{MHz}_{,} \mathrm{CDCl}_{3}$ ) $\delta 167.8,143.0,138.0,133.8,131.2,130.4,126.7,119.3,52.0,20.1$; IR (neat, $\mathrm{cm}^{-1}$ ): 1712 ; HRMS calcd for $\mathrm{C}_{11} \mathrm{H}_{12} \mathrm{O}_{2}$ : 176.0837; Found: 176.0834 .<smiles>COC(=O)/C=C/c1ccccc1Cl</smiles>

$5 c$

(E)-Methyl 3-(2-chlorophenyl)acrylate (5c). ${ }^{15}{ }^{1} \mathrm{H} \mathrm{NMR}\left(400 \mathrm{MHz}, \mathrm{CDCl}_{3}\right) \delta 8.09(\mathrm{~d}, J=16 \mathrm{~Hz}, 1 \mathrm{H})$, $7.61(\mathrm{~d}, J=7.2 \mathrm{~Hz}, 1 \mathrm{H}), 7.41(\mathrm{~d}, J=7.2 \mathrm{~Hz}, 1 \mathrm{H}), 7.31-7.25(\mathrm{~m}, 2 \mathrm{H}), 6.43$ (d, $J=16 \mathrm{~Hz}, 1 \mathrm{H}), 3.82$ (s, $3 \mathrm{H}) ;{ }^{13} \mathrm{C} \mathrm{NMR}\left(100 \mathrm{MHz}, \mathrm{CDCl}_{3}\right) \delta 167.3,141.0,135.4,133.2,131.4,130.6,128.1,127.5,120.9,52.2$; IR (neat, $\mathrm{cm}^{-1}$ ): 1714; HRMS calcd for $\mathrm{C}_{10} \mathrm{H}_{9} \mathrm{ClO}_{2}$ : 196.0291 and 198.0262; Found: 196.0289 and 198.0266.<smiles>COC(=O)/C=C/c1ccccc1F</smiles>

5d

(E)-Methyl 3-(2-fluorophenyl)acrylate (5d). ${ }^{17}{ }^{1} \mathrm{H} \mathrm{NMR}\left(400 \mathrm{MHz}, \mathrm{CDCl}_{3}\right) \delta 7.81(\mathrm{~d}, J=16 \mathrm{~Hz}, 1 \mathrm{H})$, 7.55-7.51 (m, $1 \mathrm{H}), 7.38-7.33(\mathrm{~m}, 1 \mathrm{H}), 7.18-7.07(\mathrm{~m}, 2 \mathrm{H}), 6.54(\mathrm{~d}, J=16 \mathrm{~Hz}, 1 \mathrm{H}), 3.81(\mathrm{~s}, 3 \mathrm{H}) ;{ }^{13} \mathrm{C}$ $\operatorname{NMR}\left(100 \mathrm{MHz}, \mathrm{CDCl}_{3}\right) \delta 167.6,161.8\left(\mathrm{~d}, J_{\mathrm{CF}}=252 \mathrm{~Hz}\right), 137.8\left(\mathrm{~d}, J_{\mathrm{CF}}=3 \mathrm{~Hz}\right), 132.1\left(\mathrm{~d}, J_{\mathrm{CF}}=9 \mathrm{~Hz}\right)$, $129.5\left(\mathrm{~d}, J_{\mathrm{CF}}=3 \mathrm{~Hz}\right), 124.8\left(\mathrm{~d}, J_{\mathrm{CF}}=4 \mathrm{~Hz}\right), 122.9\left(\mathrm{~d}, J_{\mathrm{CF}}=11.4 \mathrm{~Hz}\right), 120.8\left(\mathrm{~d}, J_{\mathrm{CF}}=6 \mathrm{~Hz}\right), 116.5(\mathrm{~d}$, $J_{\mathrm{CF}}=21.4 \mathrm{~Hz}$ ), 52.1; IR (neat, $\mathrm{cm}^{-1}$ ): 1714; HRMS calcd for $\mathrm{C}_{10} \mathrm{H}_{9} \mathrm{FO}_{2}: 180.0587$; Found: 180.0589 . 


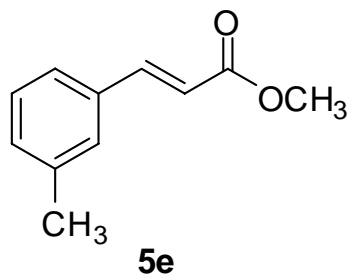

(E)-Methyl 3-(m-tolyl)acrylate (5e). ${ }^{15}{ }^{1} \mathrm{H}$ NMR $\left(400 \mathrm{MHz}, \mathrm{CDCl}_{3}\right) \delta 7.66(\mathrm{~d}, J=16 \mathrm{~Hz}, 1 \mathrm{H}), 7.33$ 7.31 (m, $2 \mathrm{H}), 7.28-7.24(\mathrm{~m}, 1 \mathrm{H}), 7.20-7.18(\mathrm{~m}, 1 \mathrm{H}), 6.42$ (d, $J=16 \mathrm{~Hz}, 1 \mathrm{H}), 3.80(\mathrm{~s}, 3 \mathrm{H}), 2.36(\mathrm{~s}, 3 \mathrm{H})$; ${ }^{13} \mathrm{C}$ NMR $\left(100 \mathrm{MHz}, \mathrm{CDCl}_{3}\right) \delta 167.9,145.4,138.9,134.8,131.5,129.2,129.1,125.7,118.0,52.0,21.7$; IR (neat, $\mathrm{cm}^{-1}$ ): 1710; HRMS calcd for $\mathrm{C}_{11} \mathrm{H}_{12} \mathrm{O}_{2}$ : 176.0837; Found: 176.0836.

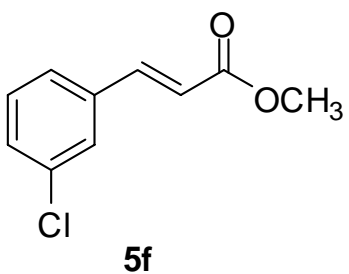

(E)-Methyl 3-(m-chlorophenyl)acrylate (5f). ${ }^{18}{ }^{1} \mathrm{H}$ NMR $\left(400 \mathrm{MHz}, \mathrm{CDCl}_{3}\right) \delta 7.62(\mathrm{~d}, J=16 \mathrm{~Hz}, 1 \mathrm{H})$, $7.50(\mathrm{~s}, 1 \mathrm{H}), 7.40-7.29(\mathrm{~m}, 2 \mathrm{H}), 6.43(\mathrm{~d}, J=16 \mathrm{~Hz}, 1 \mathrm{H}), 3.81(\mathrm{~s}, 3 \mathrm{H}) ;{ }^{13} \mathrm{C} \mathrm{NMR}\left(100 \mathrm{MHz}, \mathrm{CDCl}_{3}\right) \delta$ 167.4, 143.6, 136.7, 135.4, 130.6, 130.5, 128.2, 126.6, 119.8, 52.2; IR (neat, $\mathrm{cm}^{-1}$ ): 1716; HRMS calcd for $\mathrm{C}_{10} \mathrm{H}_{9} \mathrm{ClO}_{2}$ : 196.0291 and 198.0262; Found: 196.0293 and 198.0258.

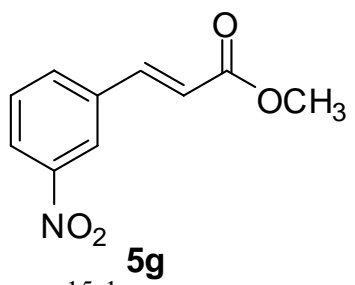

(E)-Methyl 3-(m-nitrophenyl)acrylate (5g). ${ }^{15}{ }^{1} \mathrm{H}$ NMR $\left(400 \mathrm{MHz}, \mathrm{CDCl}_{3}\right) \delta 8.37$ (s, $\left.1 \mathrm{H}\right), 8.23$ (d, $J=$ $8 \mathrm{~Hz}, 1 \mathrm{H}), 7.82(\mathrm{~d}, J=8 \mathrm{~Hz}, 1 \mathrm{H}), 7.72(\mathrm{~d}, J=16 \mathrm{~Hz}, 1 \mathrm{H}), 7.61-7.57(\mathrm{~m}, 1 \mathrm{H}), 6.56(\mathrm{~d}, J=16 \mathrm{~Hz}, 1 \mathrm{H})$, $3.84(\mathrm{~s}, 3 \mathrm{H}) ;{ }^{13} \mathrm{C}$ NMR $\left(100 \mathrm{MHz}, \mathrm{CDCl}_{3}\right) \delta 166.9,149.2,142.3,136.6,134.0,130.4,124.9,122.8$, 121.5, 52.3; IR (neat, $\mathrm{cm}^{-1}$ ): 1705, 1523, 1352; HRMS calcd for $\mathrm{C}_{10} \mathrm{H}_{9} \mathrm{NO}_{4}$ : 207.0532; Found: 207.0530 .<smiles>COC(=O)/C=C/c1ccc(C)cc1</smiles>

$5 \mathrm{~h}$

(E)-Methyl 3-(p-tolyl)acrylate (5h). ${ }^{15}{ }^{1} \mathrm{H}$ NMR $\left(400 \mathrm{MHz}, \mathrm{CDCl}_{3}\right) \delta 7.66(\mathrm{~d}, J=16 \mathrm{~Hz}, 1 \mathrm{H}), 7.41(\mathrm{~d}, J$ $=8 \mathrm{~Hz}, 2 \mathrm{H}), 7.18(\mathrm{~d}, J=8 \mathrm{~Hz}, 2 \mathrm{H}), 6.38(\mathrm{~d}, J=16 \mathrm{~Hz}, 1 \mathrm{H}), 3.79(\mathrm{~s}, 3 \mathrm{H}), 2.37(\mathrm{~s}, 3 \mathrm{H}) ;{ }^{13} \mathrm{C}$ NMR $(100$ $\mathrm{MHz}, \mathrm{CDCl}_{3}$ ) $\delta 168.0,145.3,141.1,132.1,130.0,128.5,117.2,52.0,21.8$; IR (neat, $\mathrm{cm}^{-1}$ ): 1705; HRMS calcd for $\mathrm{C}_{11} \mathrm{H}_{12} \mathrm{O}_{2}$ : 176.0837; Found: 176.0834 . 
<smiles>COC(=O)/C=C/c1ccc(OC)cc1</smiles>

$5 i$

(E)-Methyl 3-(p-methoxyphenyl)acrylate (5i). ${ }^{15}{ }^{1} \mathrm{H}$ NMR $\left(400 \mathrm{MHz}, \mathrm{CDCl}_{3}\right) \delta 7.64(\mathrm{~d}, J=16 \mathrm{~Hz}$, $1 \mathrm{H}), 7.47$ (d, $J=7 \mathrm{~Hz}, 2 \mathrm{H}), 6.90$ (d, $J=7 \mathrm{~Hz}, 2 \mathrm{H}), 6.30$ (d, $J=16 \mathrm{~Hz}, 1 \mathrm{H}), 3.83(\mathrm{~s}, 3 \mathrm{H}), 3.79$ (s, 3H); ${ }^{13} \mathrm{C} \mathrm{NMR}\left(100 \mathrm{MHz}, \mathrm{CDCl}_{3}\right) \delta 168.1,161.8,144.9,130.1,127.6,115.7,114.8,55.7,51.9$; IR (neat, $\mathrm{cm}^{-}$ 1): 1712; HRMS calcd for $\mathrm{C}_{11} \mathrm{H}_{12} \mathrm{O}_{3}$ : 192.0786; Found: 192.0784 .<smiles>COC(=O)/C=C/c1ccc(Cl)cc1</smiles>

$5 j$

(E)-Methyl 3-(p-chlorophenyl)acrylate (5j). ${ }^{15}{ }^{1} \mathrm{H}$ NMR $\left(400 \mathrm{MHz}, \mathrm{CDCl}_{3}\right) \delta 7.63(\mathrm{~d}, J=16 \mathrm{~Hz}, 1 \mathrm{H})$, $7.44(\mathrm{~d}, J=6.5 \mathrm{~Hz}, 2 \mathrm{H}), 7.35(\mathrm{~d}, J=6.5 \mathrm{~Hz}, 2 \mathrm{H}), 6.40(\mathrm{~d}, J=16 \mathrm{~Hz}, 1 \mathrm{H}), 3.80(\mathrm{~s}, 3 \mathrm{H}) ;{ }^{13} \mathrm{C}$ NMR $(100$ $\left.\mathrm{MHz}_{2} \mathrm{CDCl}_{3}\right) \delta 167.5,143.8,136.6,133.3,129.6,129.5,118.9,52.1$; IR (neat, $\mathrm{cm}^{-1}$ ): 1703; HRMS calcd for $\mathrm{C}_{10} \mathrm{H}_{9} \mathrm{ClO}_{2}$ : 196.0291 and 198.0262; Found: 196.0290 and 198.0267.<smiles>COC(=O)/C=C/c1ccc(Br)cc1</smiles>

$5 k$

(E)-Methyl 3-(p-bromophenyl)acrylate (5k). ${ }^{15}{ }^{1} \mathrm{H} \mathrm{NMR}\left(400 \mathrm{MHz}, \mathrm{CDCl}_{3}\right) \delta 7.62(\mathrm{~d}, J=16 \mathrm{~Hz}, 1 \mathrm{H})$, $7.51(\mathrm{~d}, J=8.5 \mathrm{~Hz}, 2 \mathrm{H}), 7.37(\mathrm{~d}, J=8.5 \mathrm{~Hz}, 2 \mathrm{H}), 6.42(\mathrm{~d}, J=16 \mathrm{~Hz}, 1 \mathrm{H}), 3.80(\mathrm{~s}, 3 \mathrm{H}) ;{ }^{13} \mathrm{C}$ NMR $(100$ $\left.\mathrm{MHz}_{2} \mathrm{CDCl}_{3}\right) \delta 167.5,143.8,133.8,132.5,129.8,124.9,119.0,52.1$; IR (neat, $\mathrm{cm}^{-1}$ ): 1705; HRMS calcd for $\mathrm{C}_{10} \mathrm{H}_{9} \mathrm{BrO}_{2}$ : 239.9786 and 241.9765; Found: 239.9790 and 241.9770.<smiles>COC(=O)/C=C/c1cccc2ccccc12</smiles>

(E)-Methyl 3-(naphthalen-4-yl)acrylate (5l). ${ }^{16}{ }^{5 \mathrm{l}} \mathrm{H} \mathrm{NMR}\left(400 \mathrm{MHz}, \mathrm{CDCl}_{3}\right) \delta 8.53(\mathrm{~d}, J=16 \mathrm{~Hz}, 1 \mathrm{H})$, 8.20-8.18 (m, 1H), 7.90-7.86 (m, 2H), $7.74(\mathrm{~d}, J=7 \mathrm{~Hz}, 1 \mathrm{H}), 7.60-7.46(\mathrm{~m}, 3 \mathrm{H}), 6.53(\mathrm{~d}, J=16 \mathrm{~Hz}$, $1 \mathrm{H}), 3.85(\mathrm{~s}, 3 \mathrm{H}) ;{ }^{13} \mathrm{C}$ NMR $\left(100 \mathrm{MHz}, \mathrm{CDCl}_{3}\right) \delta 167.7,142.3,134.1,132.2,131.8,130.9,129.1,127.3$, 126.6, 125.8, 125.4, 123.8, 120.9, 52.1; IR (neat, $\mathrm{cm}^{-1}$ ): 1720; HRMS calcd for $\mathrm{C}_{14} \mathrm{H}_{12} \mathrm{O}_{2}:$ 212.0837; Found: 212.0840 . 
<smiles>COC(=O)/C=C(\C)c1ccccc1</smiles>

$5 \mathrm{~m}$

(E)-Methyl 3-phenylbut-2-enoate (5m). ${ }^{19}{ }^{1} \mathrm{H}$ NMR (400 MHz, $\left.\mathrm{CDCl}_{3}\right) \delta$ 7.47-7.44 (m, 2H), 7.36-7.34 $(\mathrm{m}, 3 \mathrm{H}), 6.14(\mathrm{~s}, 1 \mathrm{H}), 3.74(\mathrm{~s}, 3 \mathrm{H}), 2.57(\mathrm{~s}, 3 \mathrm{H}) ;{ }^{13} \mathrm{C} \mathrm{NMR}\left(100 \mathrm{MHz}, \mathrm{CDCl}_{3}\right) \delta 167.7,156.3,142.6$, 129.5, 128.9, 126.7, 117.1, 51.5, 18.4; IR (neat, $\mathrm{cm}^{-1}$ ): 1710; HRMS calcd for $\mathrm{C}_{11} \mathrm{H}_{12} \mathrm{O}_{2}$ : 176.0837; Found: 176.0839.<smiles>COC(=O)/C=C(\C)c1ccc(OC)cc1</smiles>

$5 n$

(E)-Methyl 3-(4-methoxyphenyl)but-2-enoate (5n). ${ }^{20}{ }^{1} \mathrm{H} \mathrm{NMR}\left(400 \mathrm{MHz}, \mathrm{CDCl}_{3}\right) \delta 7.45(\mathrm{~d}, J=9 \mathrm{~Hz}$, $2 \mathrm{H}), 6.89(\mathrm{~d}, J=9 \mathrm{~Hz}, 2 \mathrm{H}), 6.12(\mathrm{~s}, 1 \mathrm{H}), 3.83(\mathrm{~s}, 3 \mathrm{H}), 3.74(\mathrm{~s}, 3 \mathrm{H}), 2.57(\mathrm{~s}, 3 \mathrm{H}) ;{ }^{13} \mathrm{C} \mathrm{NMR}(100 \mathrm{MHz}$, $\left.\mathrm{CDCl}_{3}\right) \delta 167.9,160.9,155.7,134.7,128.1,115.2,114.3,55.8,51.4,18.1$; IR (neat, $\mathrm{cm}^{-1}$ ): 1707; HRMS calcd for $\mathrm{C}_{12} \mathrm{H}_{14} \mathrm{O}_{3}$ : 206.0943; Found: 206.0944.<smiles>COC(=O)/C=C(\C)c1ccc(Br)cc1</smiles>

(E)-Methyl 3-(4-bromophenyl)but-2-enoate (5o). ${ }^{20}{ }^{50} \mathrm{H}$ NMR $\left(400 \mathrm{MHz}, \mathrm{CDCl}_{3}\right) \delta 7.48(\mathrm{~d}, J=9 \mathrm{~Hz}$, $2 \mathrm{H}), 7.32(\mathrm{~d}, J=9 \mathrm{~Hz}, 2 \mathrm{H}), 6.11(\mathrm{~s}, 1 \mathrm{H}), 3.75(\mathrm{~s}, 3 \mathrm{H}), 2.54(\mathrm{~s}, 3 \mathrm{H}) ;{ }^{13} \mathrm{C} \mathrm{NMR}\left(100 \mathrm{MHz}, \mathrm{CDCl}_{3}\right) \delta$ 167.4, 154.8, 141.4, 132.1, 128.3, 123.7, 117.5, 51.6, 18.2; IR (neat, $\mathrm{cm}^{-1}$ ): 1705; HRMS calcd for $\mathrm{C}_{11} \mathrm{H}_{11} \mathrm{BrO}_{2}$ : 253.9942 and 255.9922; Found: 253.9940 and 255.9928.

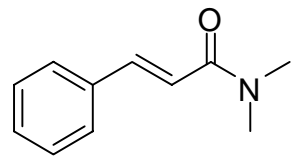

$5 p$

N,N-Dimethylcinnamamide (5p). ${ }^{22}{ }^{1} \mathrm{H}$ NMR (400 MHz, $\left.\mathrm{CDCl}_{3}\right) \delta 7.67(\mathrm{~d}, J=16 \mathrm{~Hz}, 1 \mathrm{H})$, 7.54-7.52 $(\mathrm{m}, 2 \mathrm{H}), 7.37-7.35(\mathrm{~m}, 3 \mathrm{H}), 6.89(\mathrm{~d}, J=16 \mathrm{~Hz}, 1 \mathrm{H}), 3.17(\mathrm{~s}, 3 \mathrm{H}), 3.07(\mathrm{~s}, 3 \mathrm{H}) ;{ }^{13} \mathrm{C} \mathrm{NMR}(100 \mathrm{MHz}$, $\left.\mathrm{CDCl}_{3}\right) \delta 167.2,142.8,135.8,130.0,129.2,128.2,117.8,37.9,36.4$; IR (neat, $\mathrm{cm}^{-1}$ ): 1649; HRMS calcd for $\mathrm{C}_{11} \mathrm{H}_{13} \mathrm{NO}$ : 175.0997; Found: 175.1001 . 
<smiles>COc1ccc(/C=C/C(=O)N(C)C)cc1</smiles>

(E)-3-(4-Methoxyphenyl)-N,N-dimethylacrylamide (5q). ${ }^{22} \mathrm{H} \mathrm{NMR}\left(400 \mathrm{MHz}, \mathrm{CDCl}_{3}\right) \delta 7.63(\mathrm{~d}, J=$ $16 \mathrm{~Hz}, 1 \mathrm{H}), 7.47(\mathrm{~d}, J=9 \mathrm{~Hz}, 2 \mathrm{H}), 6.88(\mathrm{~d}, J=9 \mathrm{~Hz}, 2 \mathrm{H}), 6.76(\mathrm{~d}, J=16 \mathrm{~Hz}, 1 \mathrm{H}), 3.82(\mathrm{~s}, 3 \mathrm{H}), 3.15$ (s, 3H), $3.06(\mathrm{~s}, 3 \mathrm{H}) ;{ }^{13} \mathrm{C} \mathrm{NMR}\left(100 \mathrm{MHz}, \mathrm{CDCl}_{3}\right) \delta 167.4,161.2,142.4,129.7,128.5,115.3,114.6,55.7$, 37.8, 36.3; IR (neat, $\mathrm{cm}^{-1}$ ): 1648; HRMS calcd for $\mathrm{C}_{12} \mathrm{H}_{15} \mathrm{NO}_{2}$ : 205.1103; Found: 205.1101 .

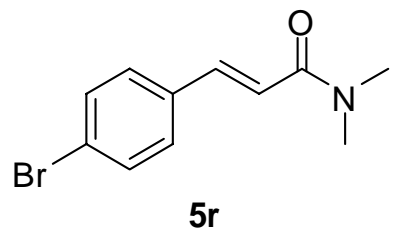

(E)-3-(4-Bromophenyl)-N,N-dimethylacrylamide (5r). ${ }^{24}{ }^{1} \mathrm{H}$ NMR $\left(400 \mathrm{MHz}, \mathrm{CDCl}_{3}\right) \delta 7.59(\mathrm{~d}, J=$ $16 \mathrm{~Hz}, 1 \mathrm{H}), 7.49(\mathrm{~d}, J=9 \mathrm{~Hz}, 2 \mathrm{H}), 7.38(\mathrm{~d}, J=9 \mathrm{~Hz}, 2 \mathrm{H}), 6.88(\mathrm{~d}, J=16 \mathrm{~Hz}, 1 \mathrm{H}), 3.17$ (s, 3H), 3.07 (s, $3 \mathrm{H}) ;{ }^{13} \mathrm{C} \mathrm{NMR}\left(100 \mathrm{MHz}, \mathrm{CDCl}_{3}\right) \delta 166.8,141.4,134.7,132.4,129.6,124.0,118.5,37.8,36.4$; IR (neat, $\mathrm{cm}^{-1}$ ): 1647; HRMS calcd for $\mathrm{C}_{11} \mathrm{H}_{12} \mathrm{BrNO}$ : 253.0102 and 255.0082; Found: 253.0100 and 255.0079.

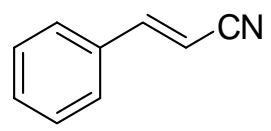

5s

Cinnamonitrile (5s). ${ }^{16}{ }^{1} \mathrm{H}$ NMR $\left(400 \mathrm{MHz}, \mathrm{CDCl}_{3}\right) \delta 7.46-7.48(\mathrm{~m}, 6 \mathrm{H}), 5.88(\mathrm{~d}, \mathrm{~J}=17 \mathrm{~Hz}, 1 \mathrm{H}) ;{ }^{13} \mathrm{C}$ NMR $\left(100 \mathrm{MHz}, \mathrm{CDCl}_{3}\right) \delta 151.0,134.0,131.7,129.6,127.8,118.6,96.8$; IR (neat, $\left.\mathrm{cm}^{-1}\right): 2216$; HRMS calcd for $\mathrm{C}_{9} \mathrm{H}_{7} \mathrm{~N}$ : 129.0578; Found: 129.0580 .

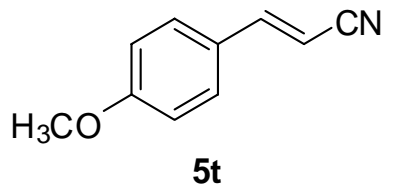

(E)-3-(4-Methoxyphenyl)acrylonitrile (5t). ${ }^{16}{ }^{1} \mathrm{H}$ NMR $\left(400 \mathrm{MHz}, \mathrm{CDCl}_{3}\right) \delta 7.40(\mathrm{~d}, \mathrm{~J}=9 \mathrm{~Hz}, 2 \mathrm{H})$, $7.33(\mathrm{~d}, J=17 \mathrm{~Hz}, 1 \mathrm{H}), 6.91(\mathrm{~d}, J=9 \mathrm{~Hz}, 2 \mathrm{H}), 5.72(\mathrm{~d}, J=17 \mathrm{~Hz}, 1 \mathrm{H}) ;{ }^{13} \mathrm{C} \mathrm{NMR}\left(100 \mathrm{MHz}, \mathrm{CDCl}_{3}\right) \delta$ 162.5, 150.5, 129.5, 126.8, 119.1, 114.9, 93.8, 55.9; IR (neat, $\mathrm{cm}^{-1}$ ): 2212; HRMS calcd for $\mathrm{C}_{10} \mathrm{H}_{9} \mathrm{NO}$ : 159.0684; Found: 159.0681.

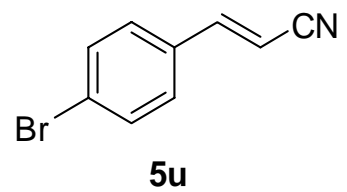

(E)-3-(4-Bromophenyl)acrylonitrile (5u). ${ }^{16}{ }^{1} \mathrm{H} \mathrm{NMR}\left(400 \mathrm{MHz}, \mathrm{CDCl}_{3}\right) \delta 7.55(\mathrm{~d}, \mathrm{~J}=8 \mathrm{~Hz}, 2 \mathrm{H}), 7.34$ $(\mathrm{d}, J=17 \mathrm{~Hz}, 1 \mathrm{H}), 7.31(\mathrm{~d}, J=8 \mathrm{~Hz}, 2 \mathrm{H}), 5.88(\mathrm{~d}, J=17 \mathrm{~Hz}, 1 \mathrm{H}) ;{ }^{13} \mathrm{C} \mathrm{NMR}\left(100 \mathrm{MHz}, \mathrm{CDCl}_{3}\right) \delta 149.7$, 132.8, 129.1, 126.1, 118.2, 97.5; IR (neat, $\mathrm{cm}^{-1}$ ): 2216; HRMS calcd for $\mathrm{C}_{9} \mathrm{H}_{6} \mathrm{BrN}$ : 206.9684 and 208.9663; Found: 206.9687 and 208.9660. 
${ }^{1} \mathrm{H}$ NMR spectrum of the crude reaction solution resulting from the coupling of 1 a with $2 \mathrm{f}$ in $d_{6^{-}}$ acetone:

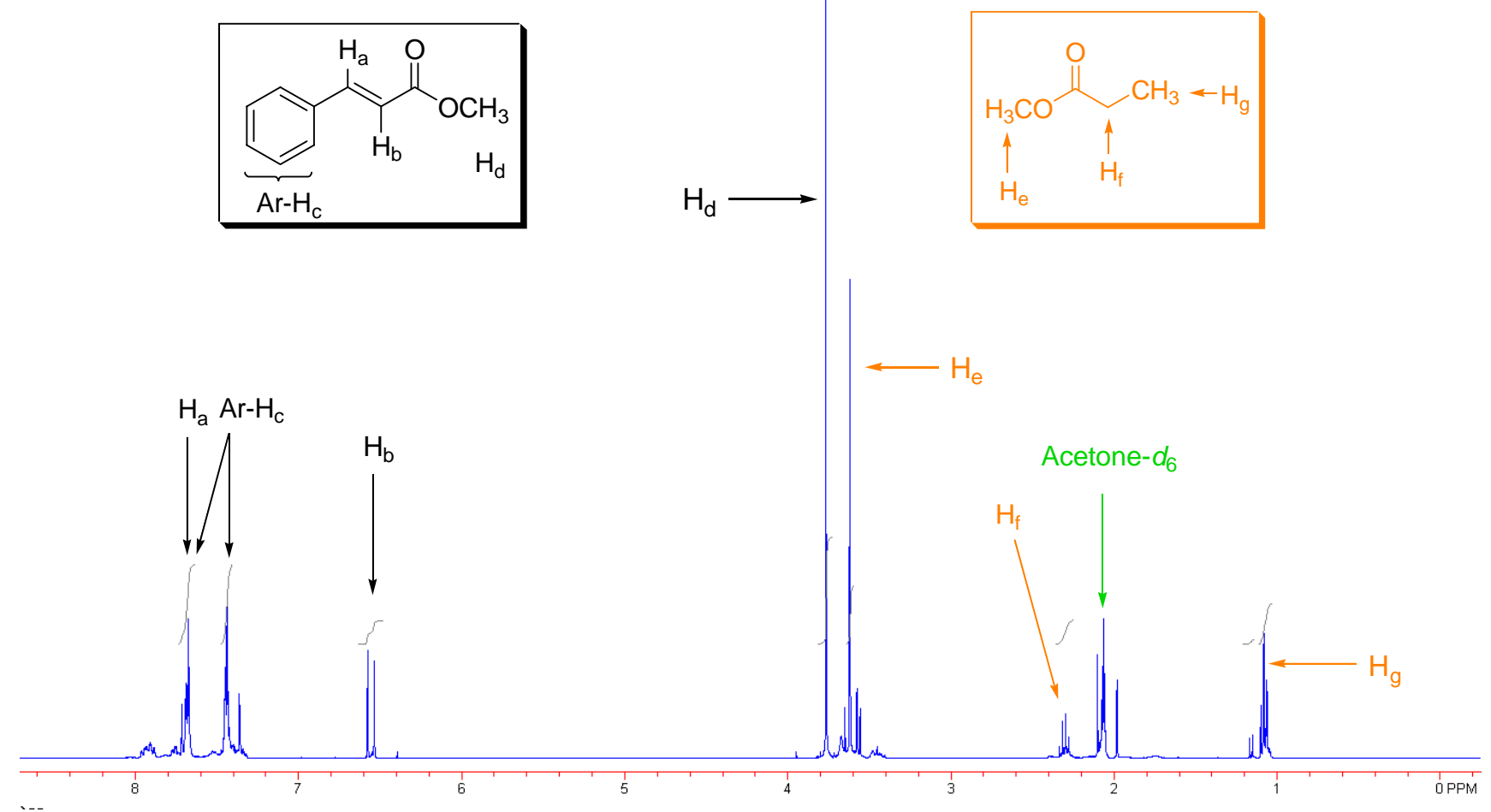

${ }^{1} \mathrm{H}$ NMR spectrum of the crude reaction solution resulting from the coupling of 1a with $2 \mathrm{a}$ in $d_{6^{-}}$ acetone:

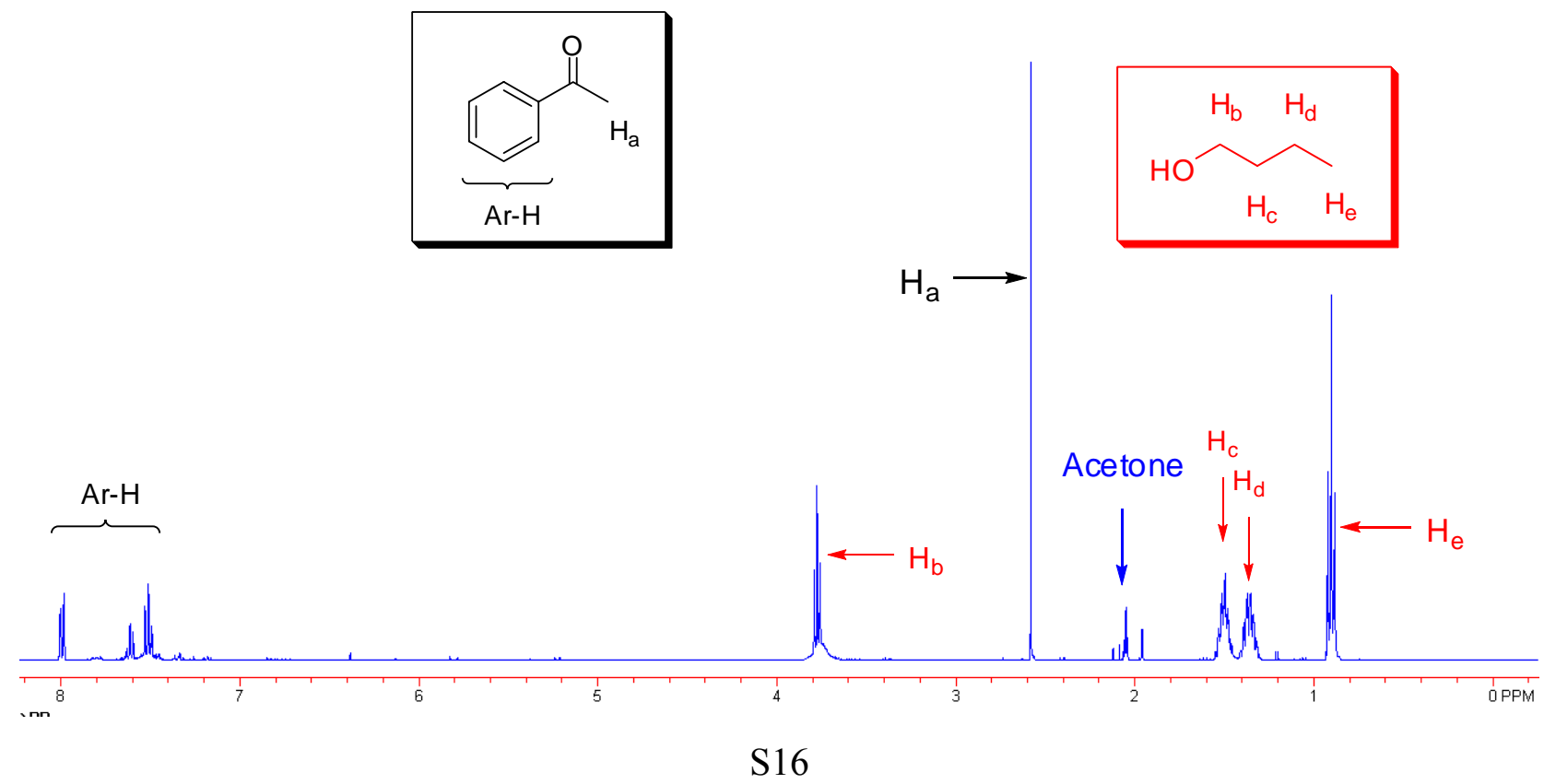




\section{References}

1. Mo, J.; Xu, L.; Xiao, J. J. Am. Chem. Soc. 2005, 127, 751.

2. $\quad$ Fujita, K.-I.; Tanino, N.; Yamaguchi, R. Org. Lett. 2007, 9, 109.

3. Graetz, B.; Rychnovsky, S.; Leu, W.-H.; Farmer, P.; Lin, R. Tetrahedron Asymm. 2005, 16, 3584.

4. Cornell, C. N.; Sigman, M. S. J. Am. Chem. Soc. 2005, 127, 2796.

5. $\quad$ Scheiper, B.; Bonnekessel, M.; Krause, H.; Fürstner, A. J. Org. Chem. 2004, 69, 3943.

6. $\quad$ Kobayashi, S.; Moriwaki, M.; hachiya, I. Bull. Chem. Soc. Jpn. 1997, 70, 267.

7. Thérien, T.; Gauthier, J. Y.; Leblanc, Y.; Léger, S.; Perrier, H.; Prasit, P.; Wang, Z. Synthesis 2001, 1778.

8. Wang, Z.; Bennett, E. M.; Wilson, D. J.; Salomon, C.; Vince, R. J. Med. Chem. 2007, 50, 3416.

9. Liu, S.; Berry, N.; Thomson, N.; Pettman, A.; Hyder, Z.; Mo, J.; Xiao, J. J. Org. Chem. 2006, 71, 7467.

10. Wang, Q. L.; Ma, Y.; Ji, X.; Yan, H.; Qiu, Q. Chem. Commun. 1995, 2307.

11. Takemiya, A.; Hartwig, J. F. J. Am. Chem. Soc. 2006, 128, 14800.

12. Tanner, D. D.; Chen, J. J.; Luelo, C.; Peters, P. M. J. Am. Chem. Soc. 1992, 114, 713.

13. Ali, B. E.; Tijani, J.; Fettouhi, M. J. Mol. Catal. A-Chem. 2005, 230, 9.

14. Gazzard, L. J.; Motherwell, W. B.; Sandham, D. A. J. Chem. Soc., Perkin Trans. 1, 1999, 979.

15. Dai, M.; Liang, B.; Wang, C.; Chen, J.; Yang, Z. Org. Lett. 2004, 6, 221.

16. Andrus, M. B.; Song, C.; Zhang, J. Org. Lett. 2002, 4, 2079.

17. Karimi, B.; Enders, D. Org. Lett. 2006, 8, 1237.

18. Tchilibon, S.; Kim, S.-K.; Gao, Z.-G.; Harris, B. A.; Blaustein, J. B.; Gross, A. S.; Duong, H. T.; Melman, N.; Jacobson, K. A. Bioorg. Med. Chem. 2004, 12, 2021.

19. Yang, D.; Chen, Y.-C.; Zhu, N.-Y. Org. Lett. 2004, 6, 1577.

20. Tang, W.; Wang, W.; Zhang, X. Angew. Chem. Int. Ed. 2003, 42, 943.

21. Kagabu, S.; Shimizu, Y.; Ito, C.; Moriya, K. Synthesis 1992, 830.

22. Zou, G.; Huang, W.; Xiao, Y.; Tang, J. New J. Chem. 2006, 30, 803.

23. Lautens, M.; Mancuso, J.; Grover, H. Synthesis 2004, 2006.

24. Sugihara, T.; Satoh, T.; Miura, M. Tetrahedron Lett. 2005, 46, 8269. 

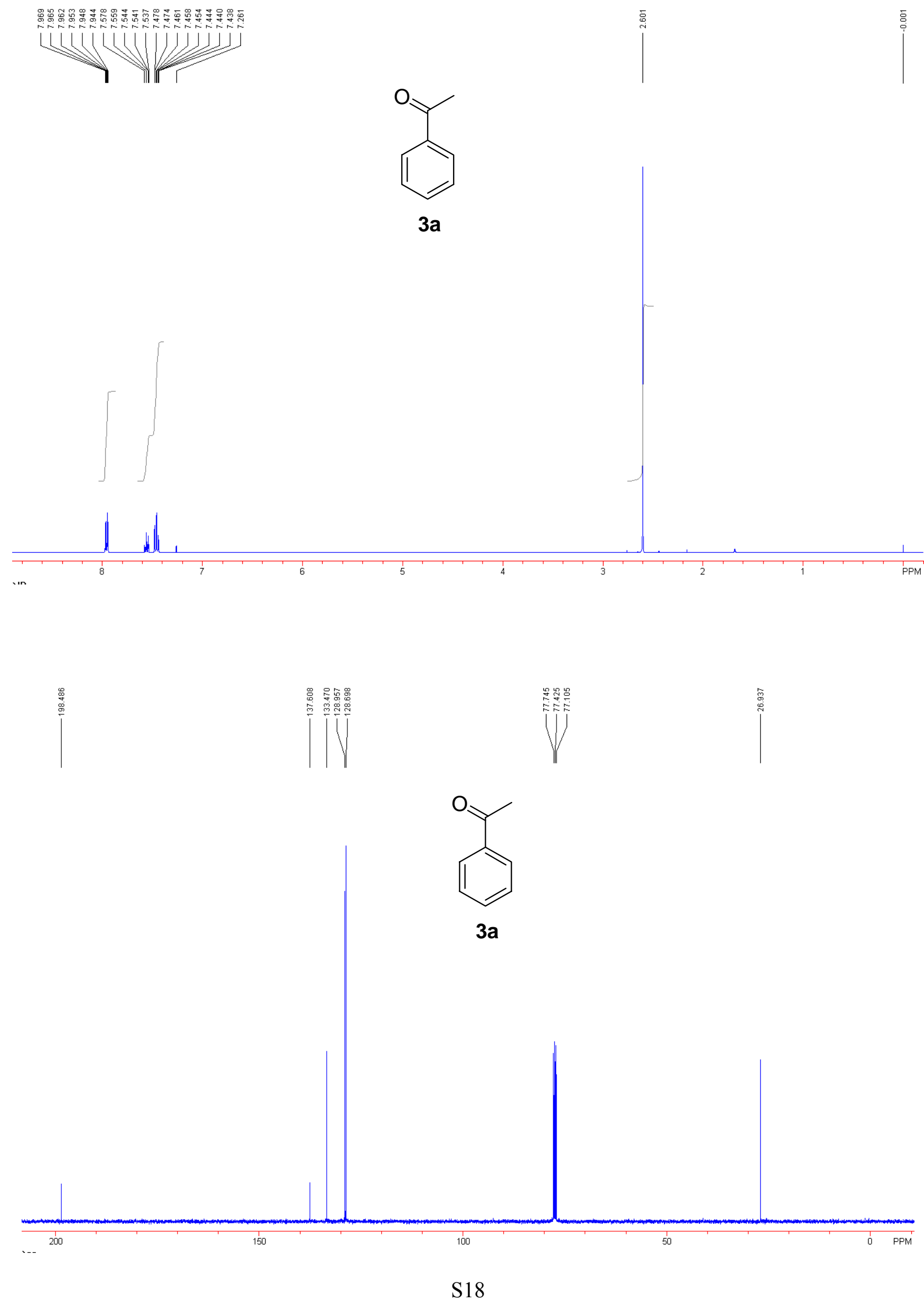

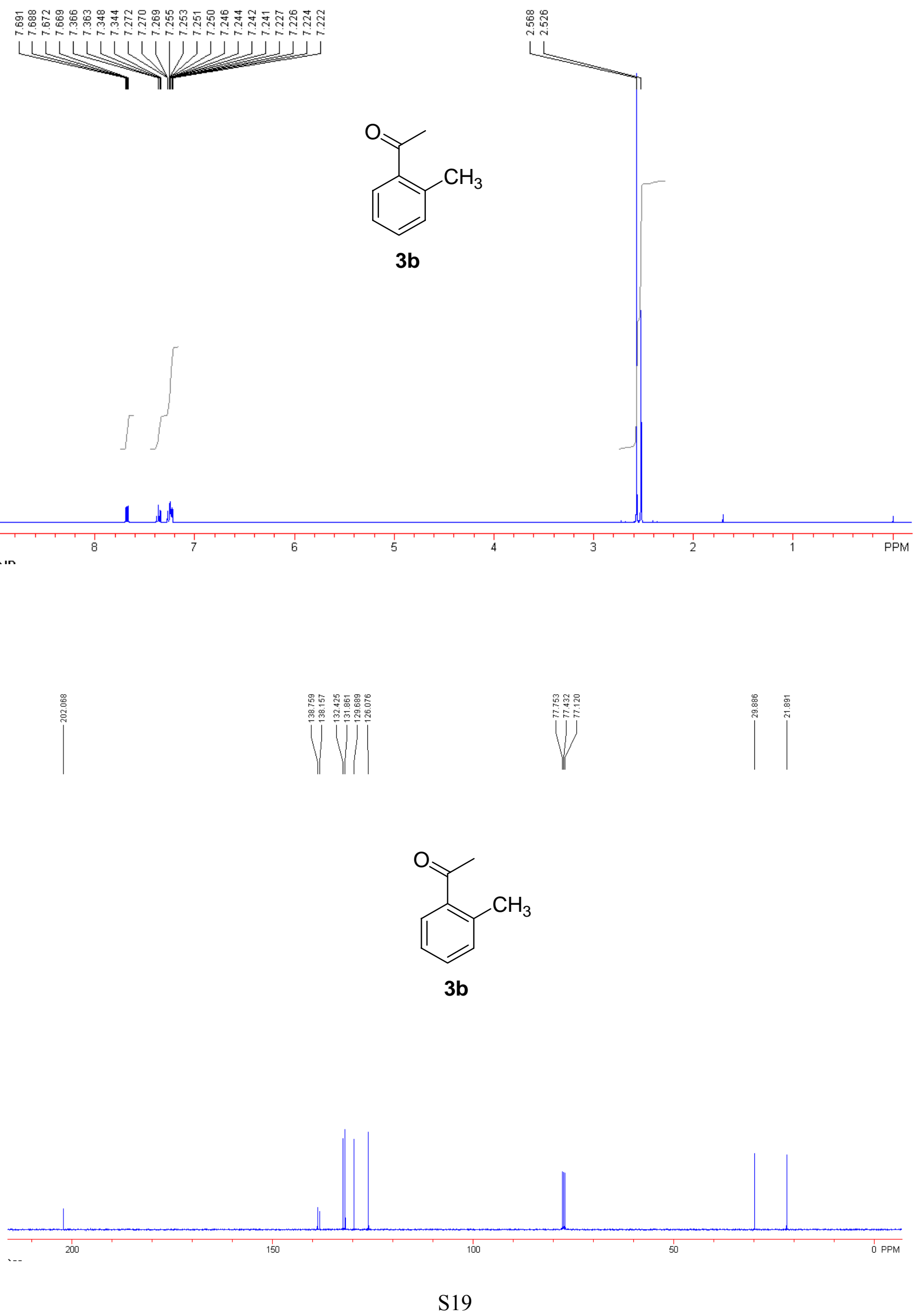

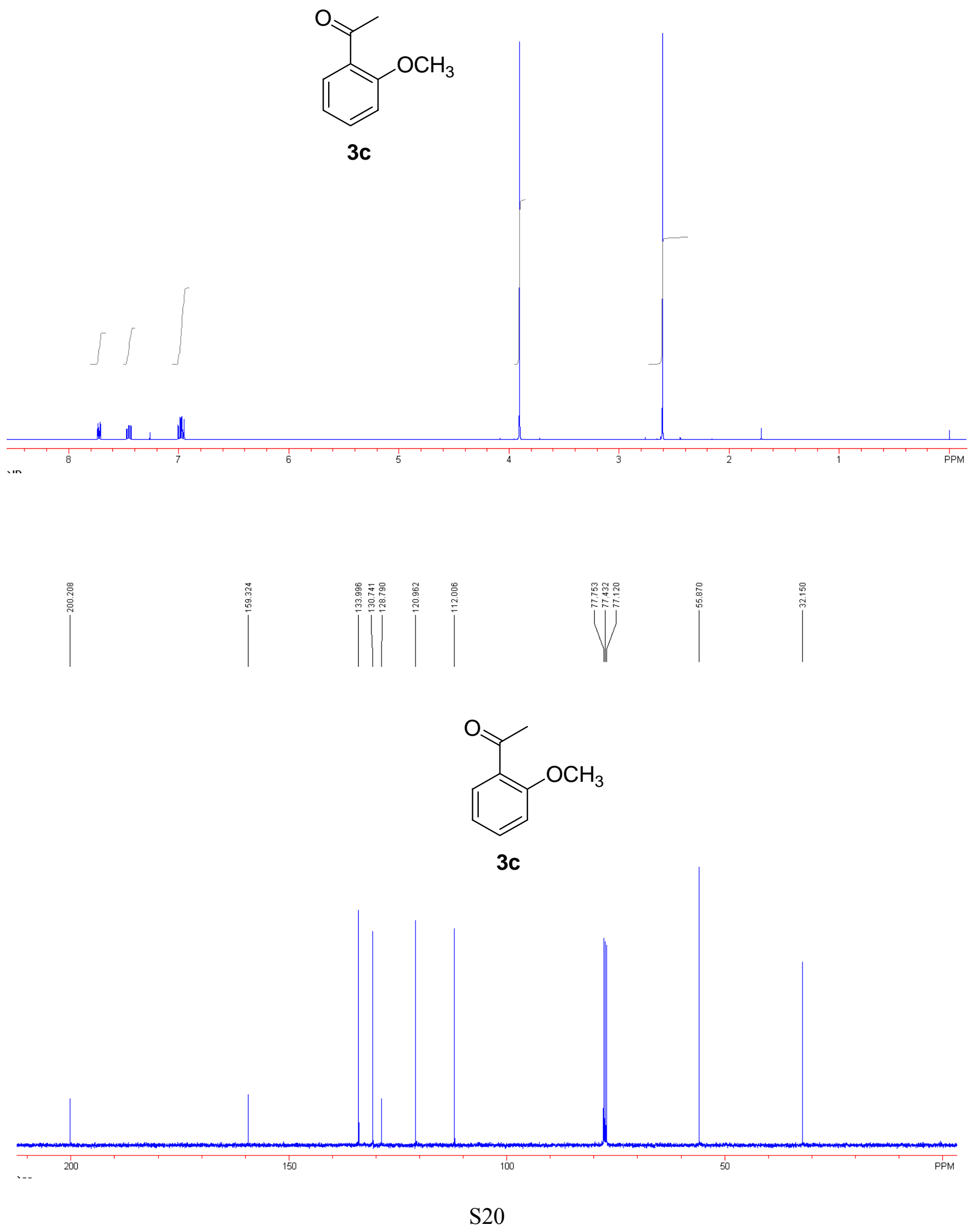

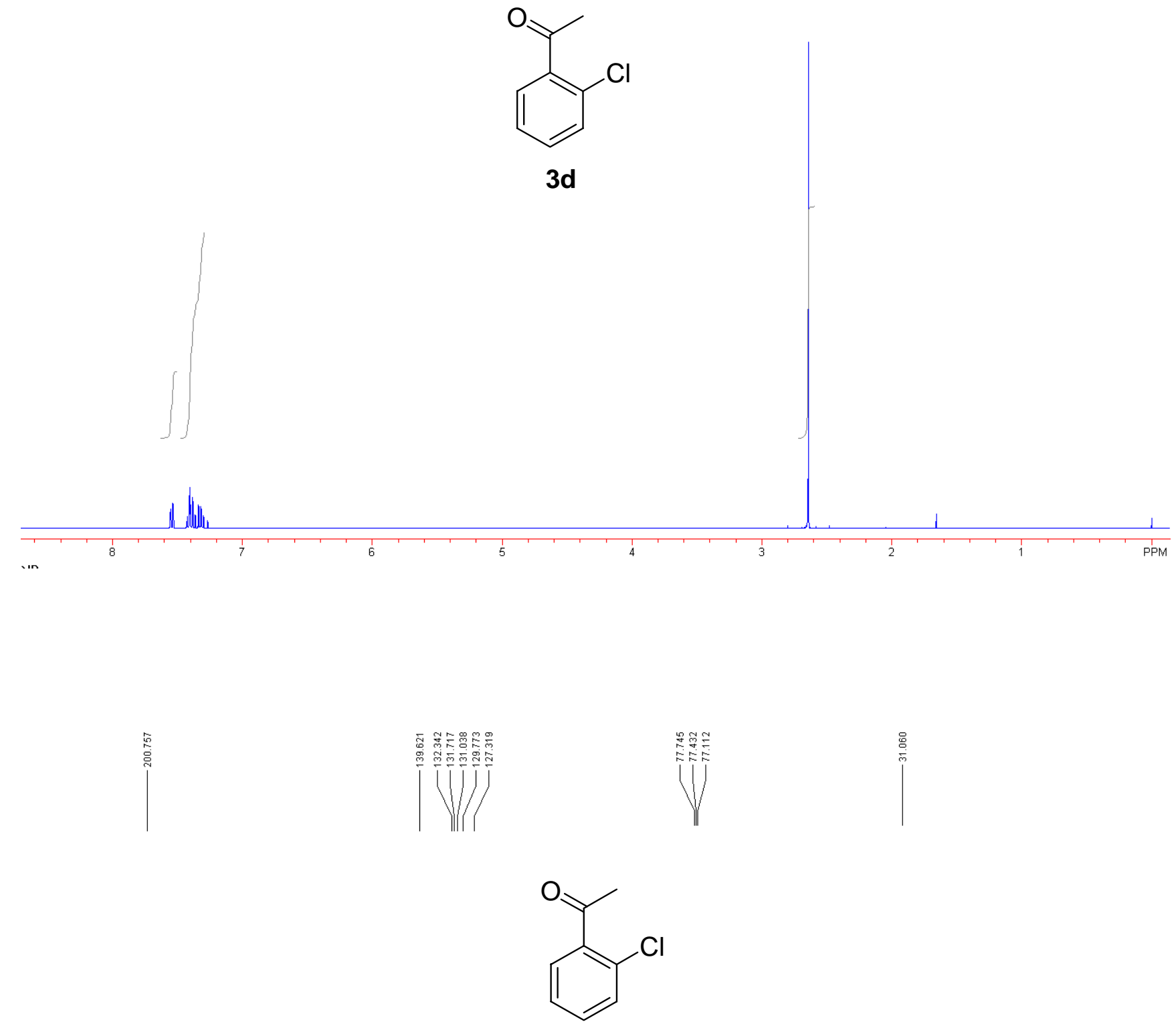

3d

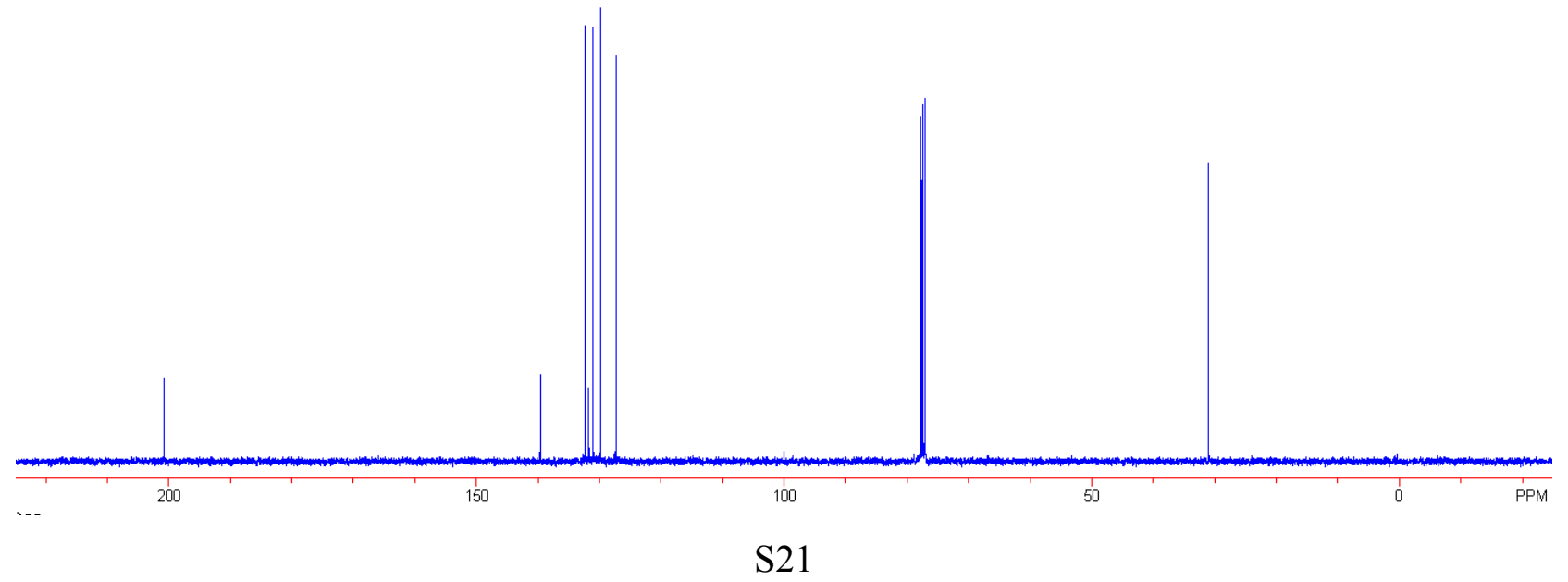



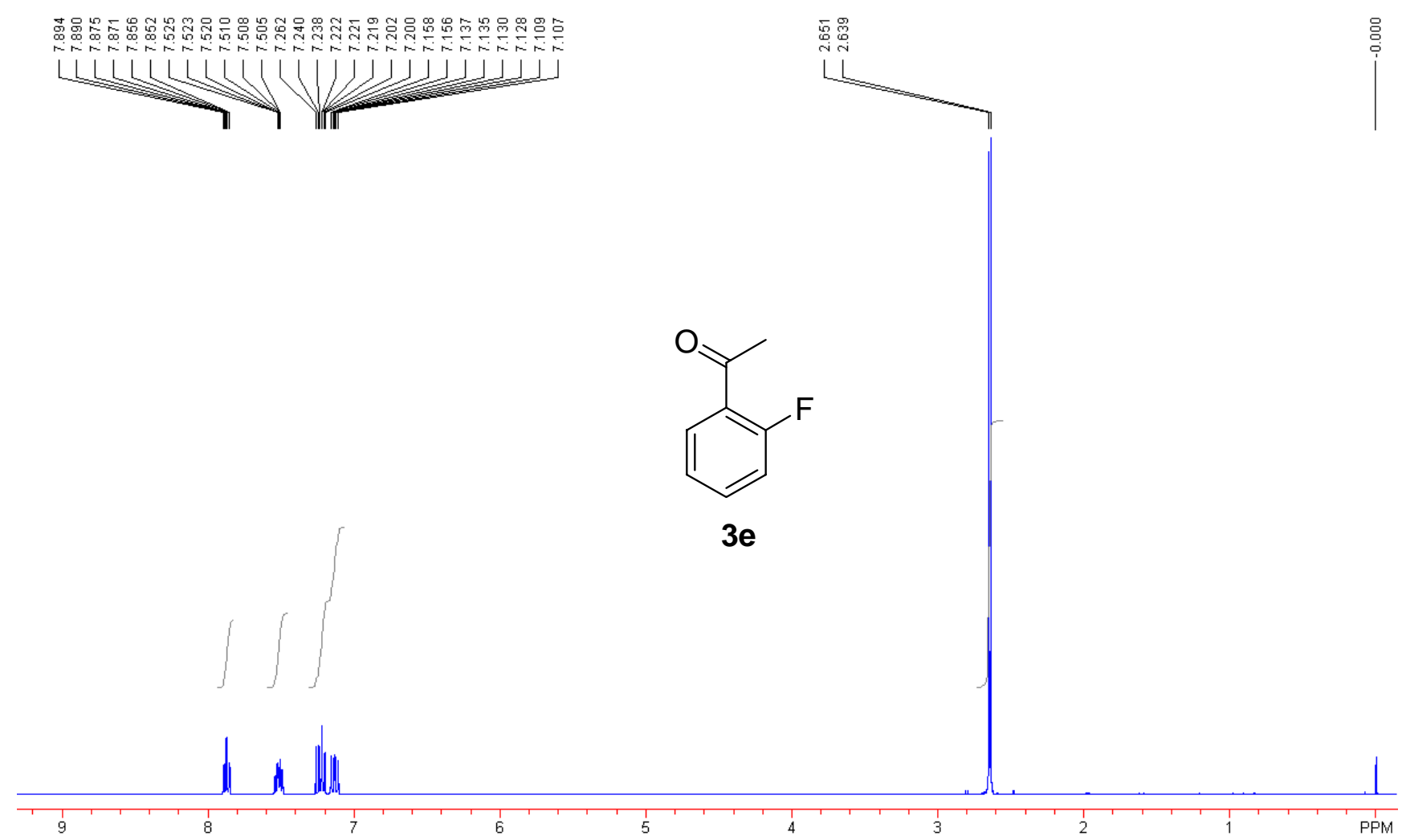<smiles>CC(=O)c1ccccc1F</smiles>

$3 e$

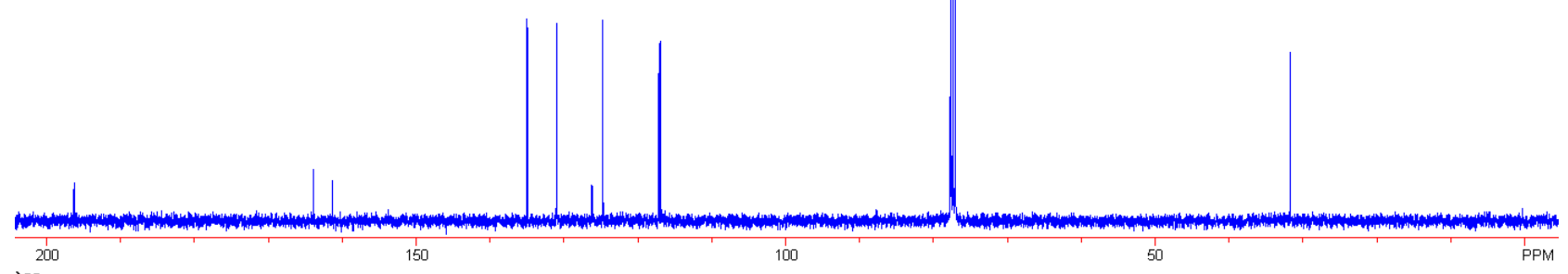



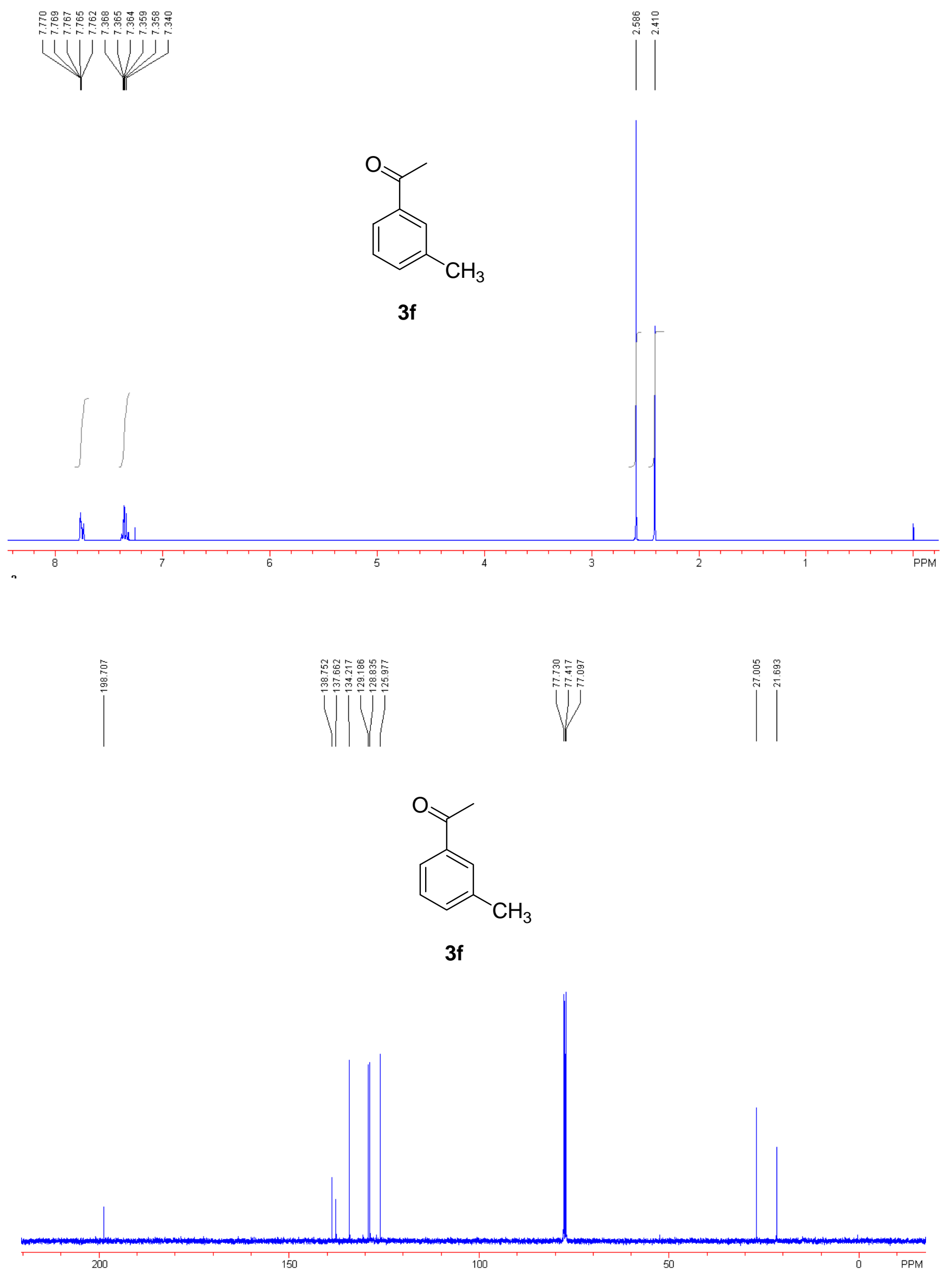


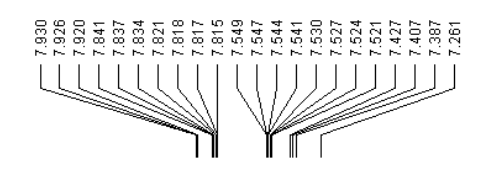

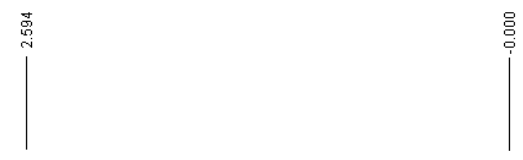
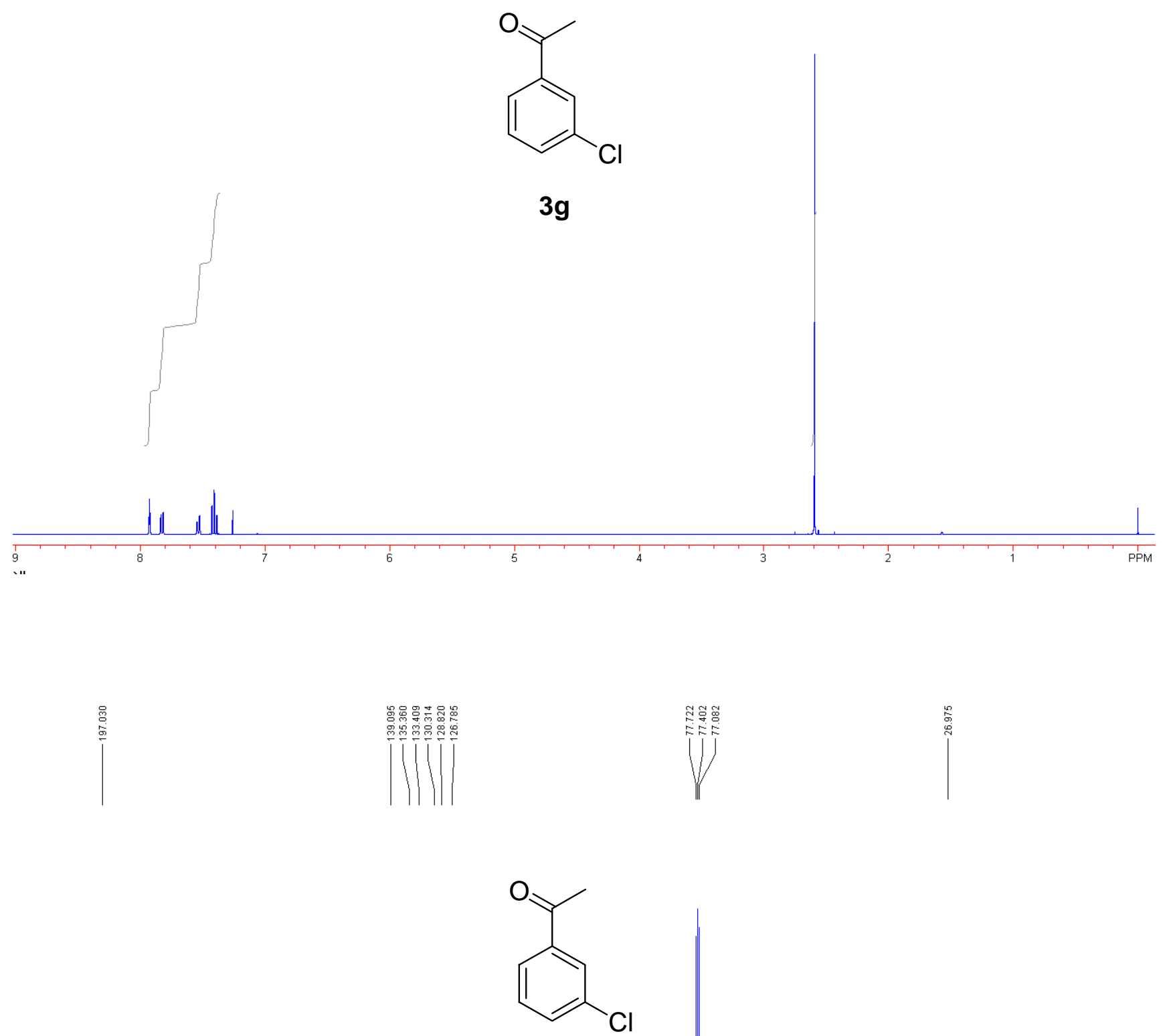

$3 g$

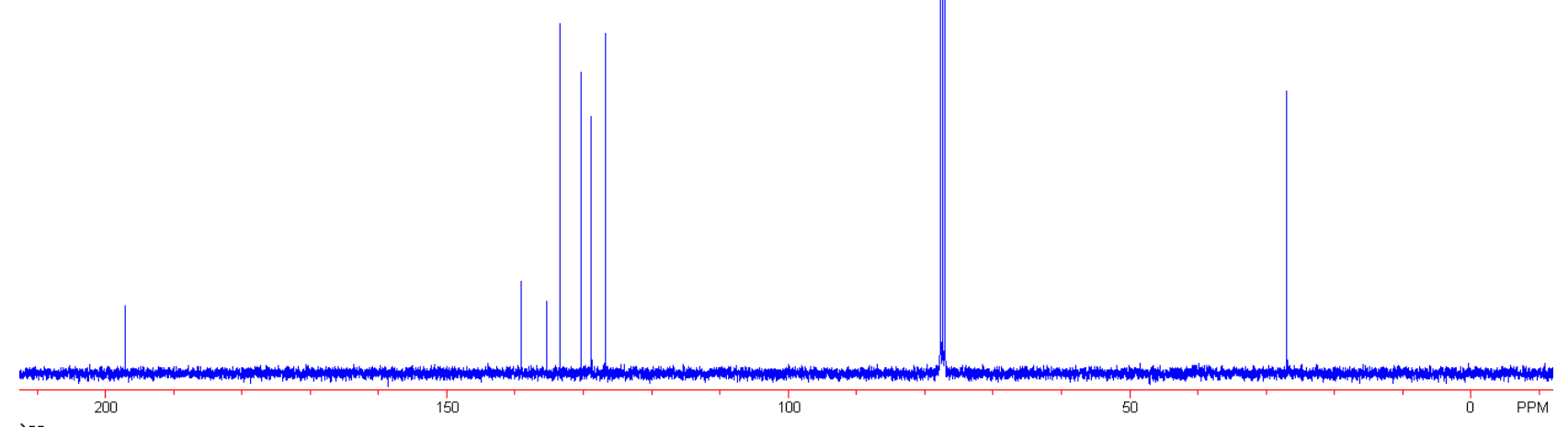



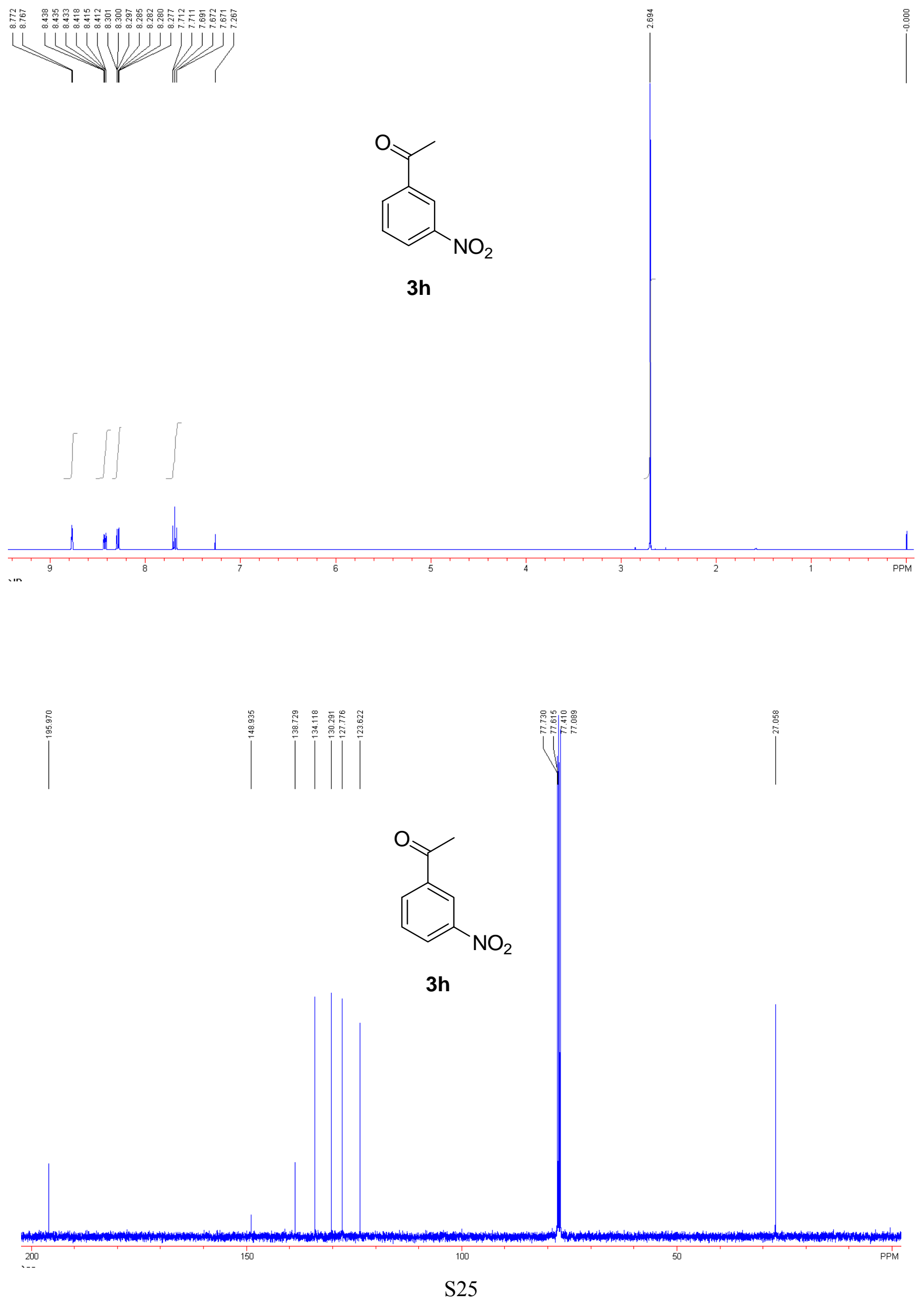


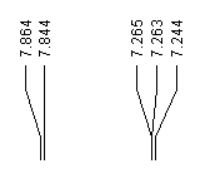

II
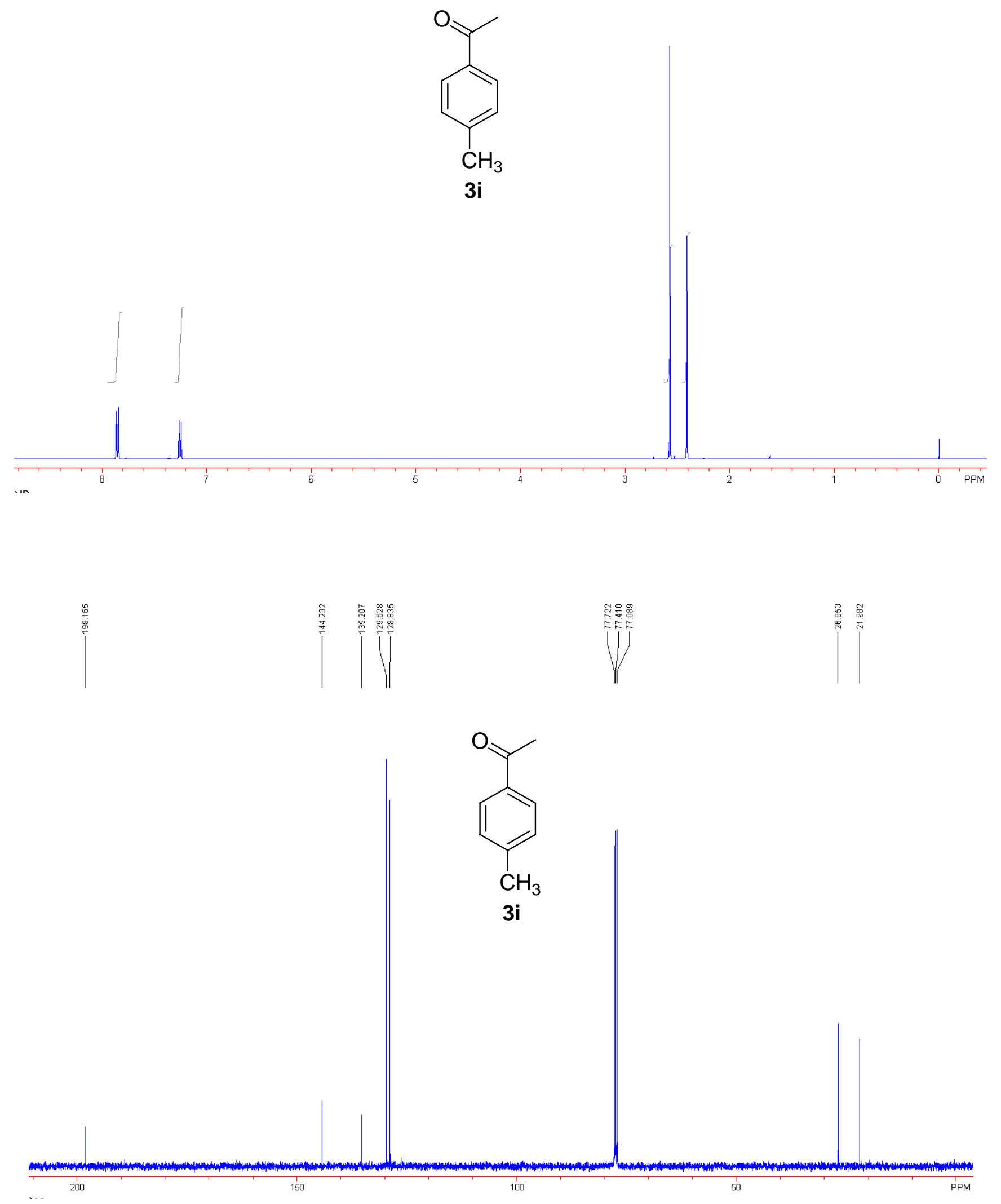

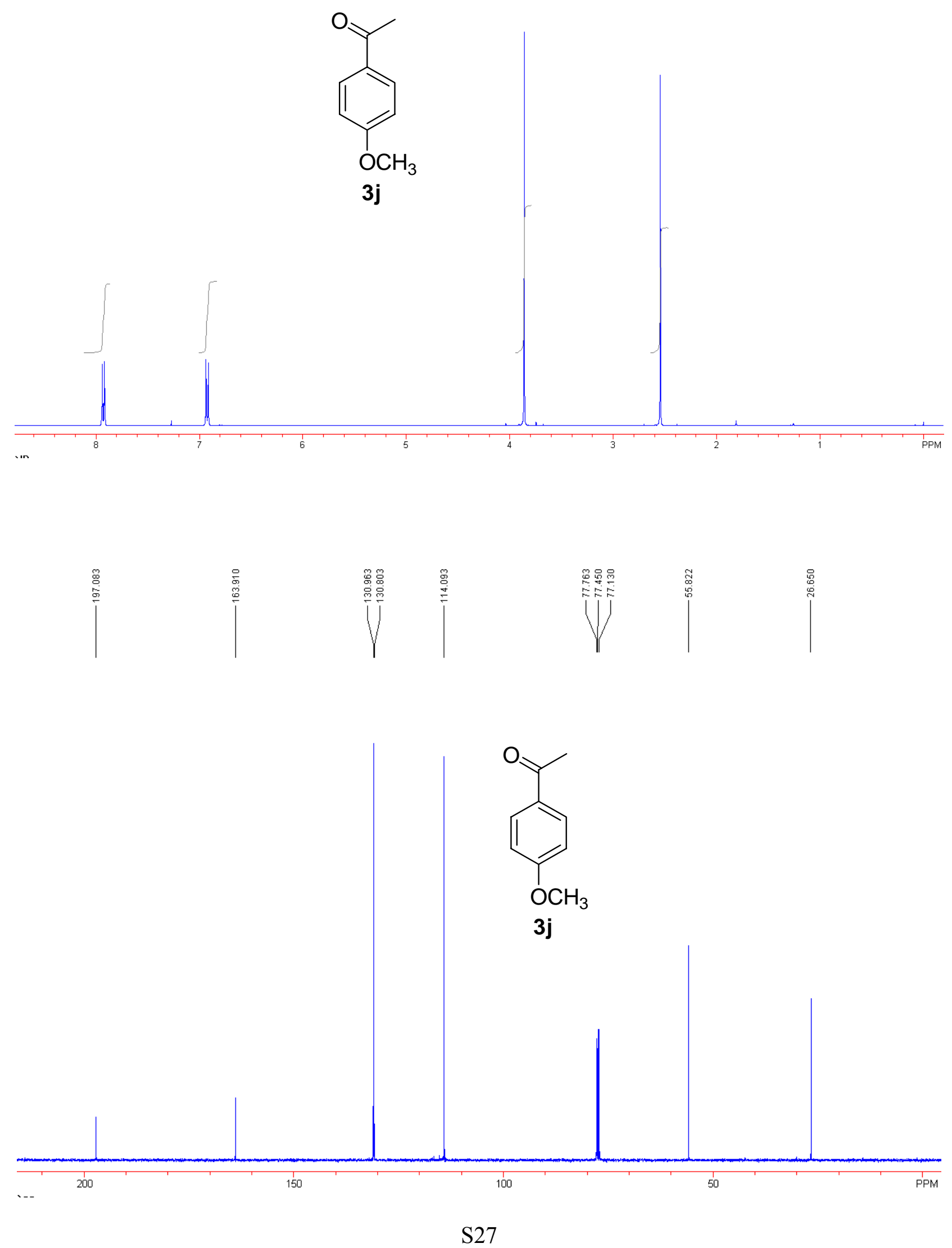

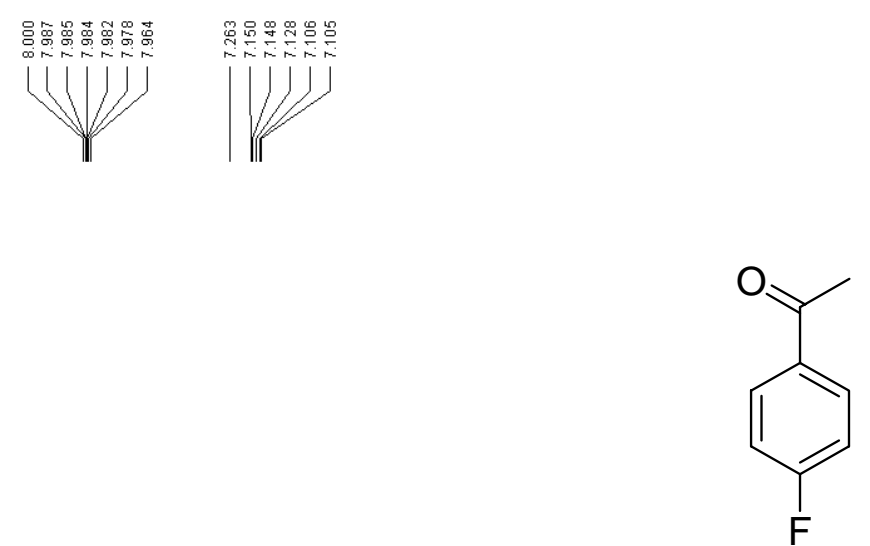

3k
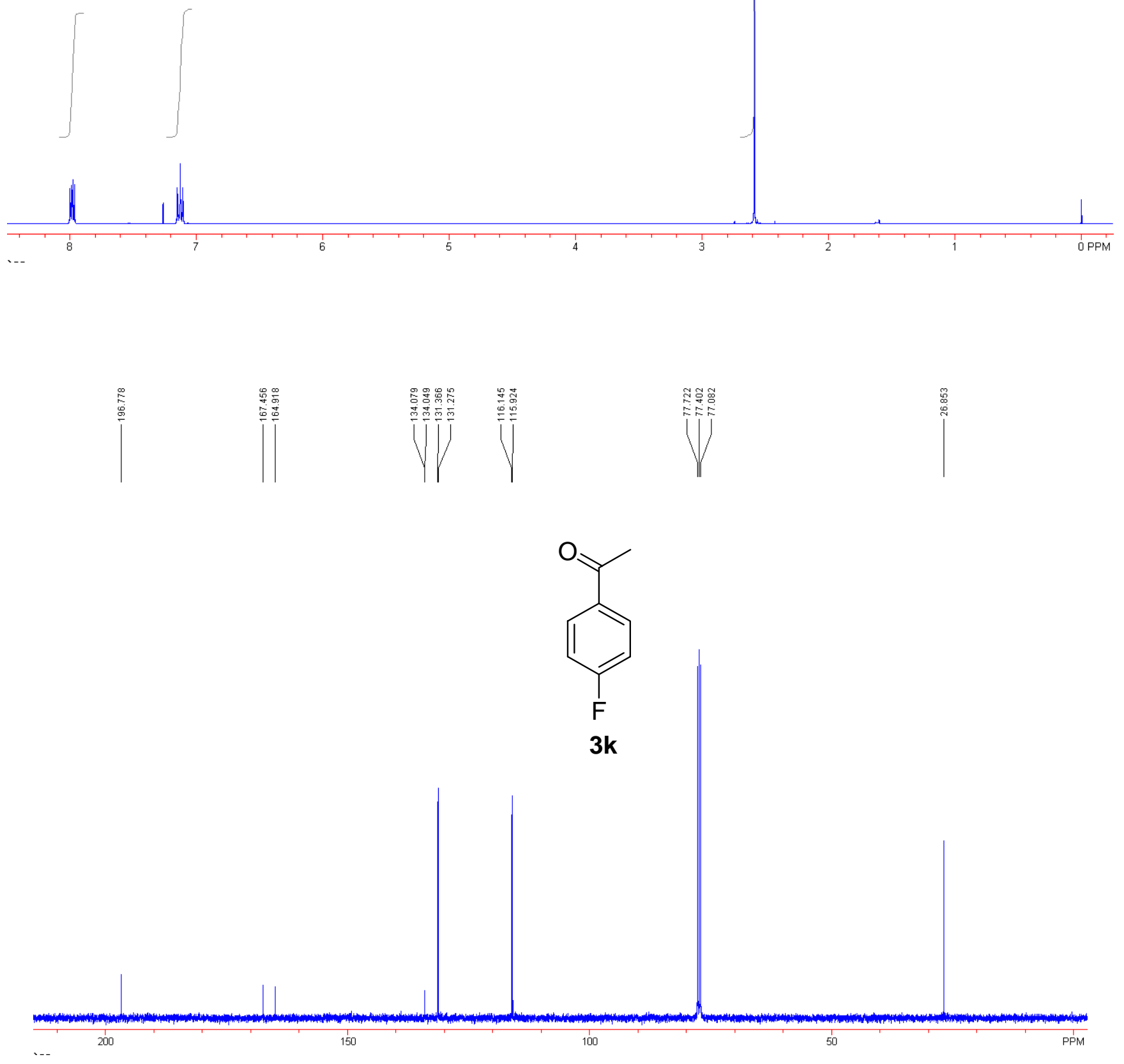

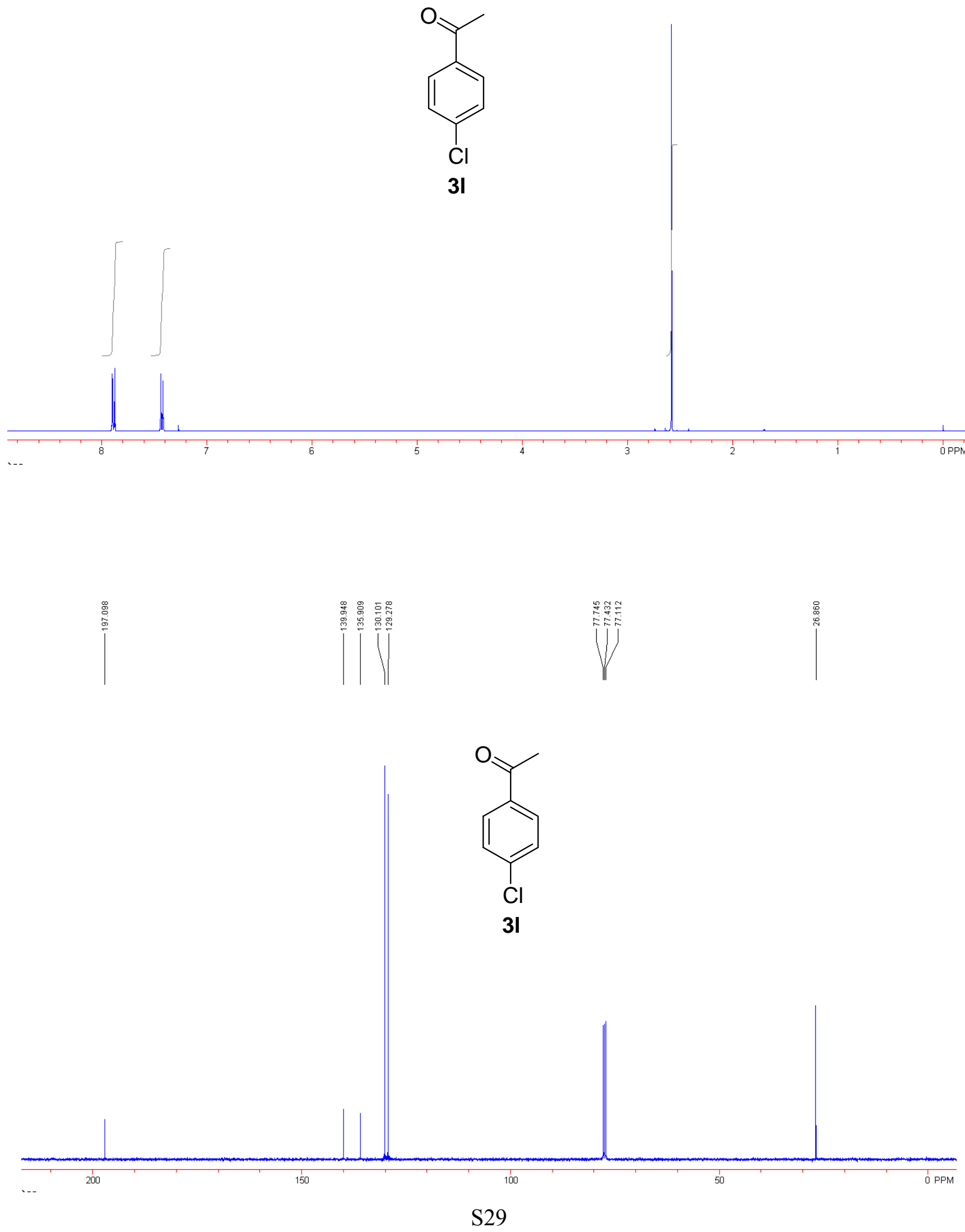


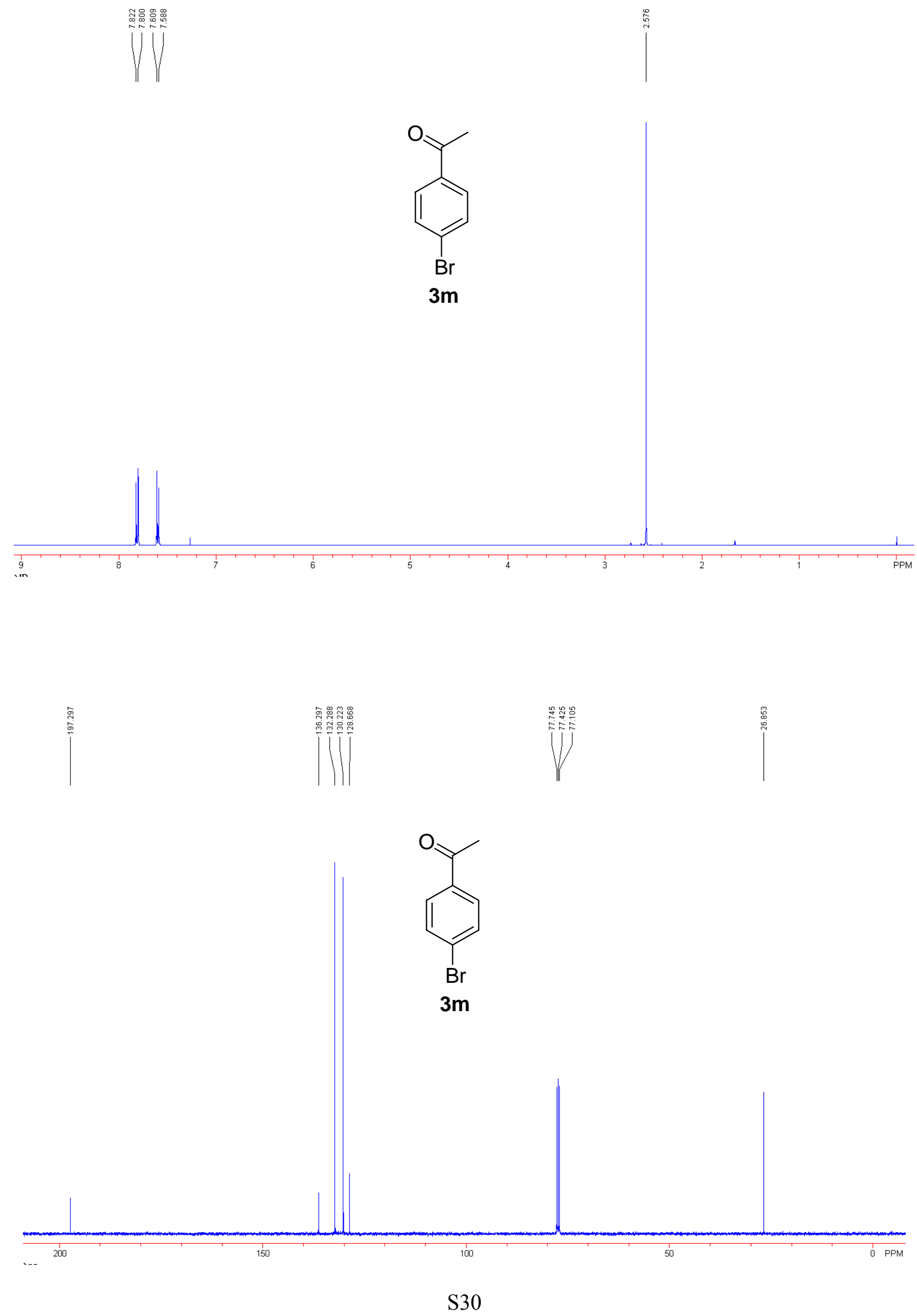




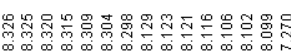

WU

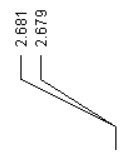<smiles>CC(=O)c1ccc([N+](=O)[O-])cc1</smiles>

3n
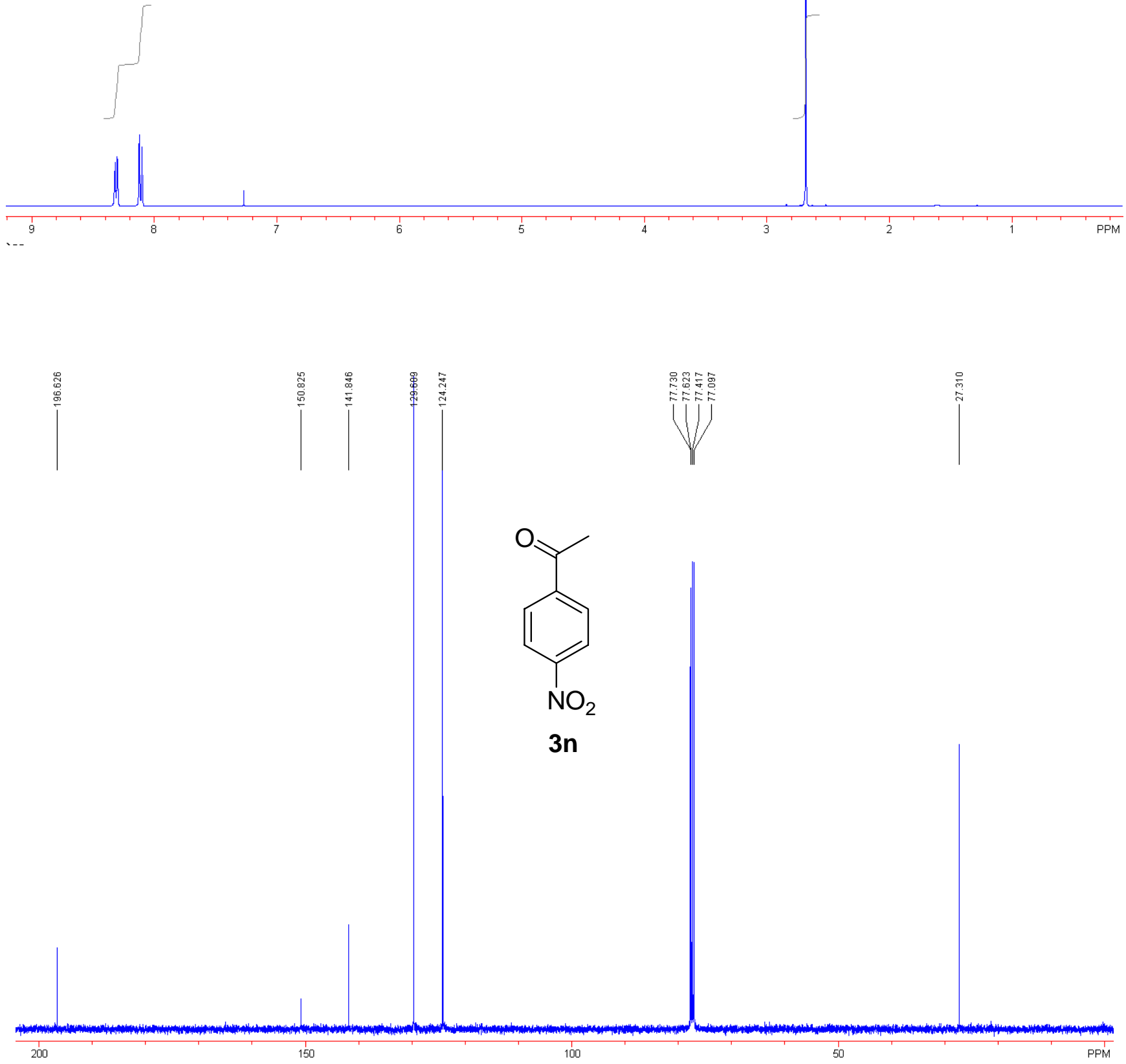

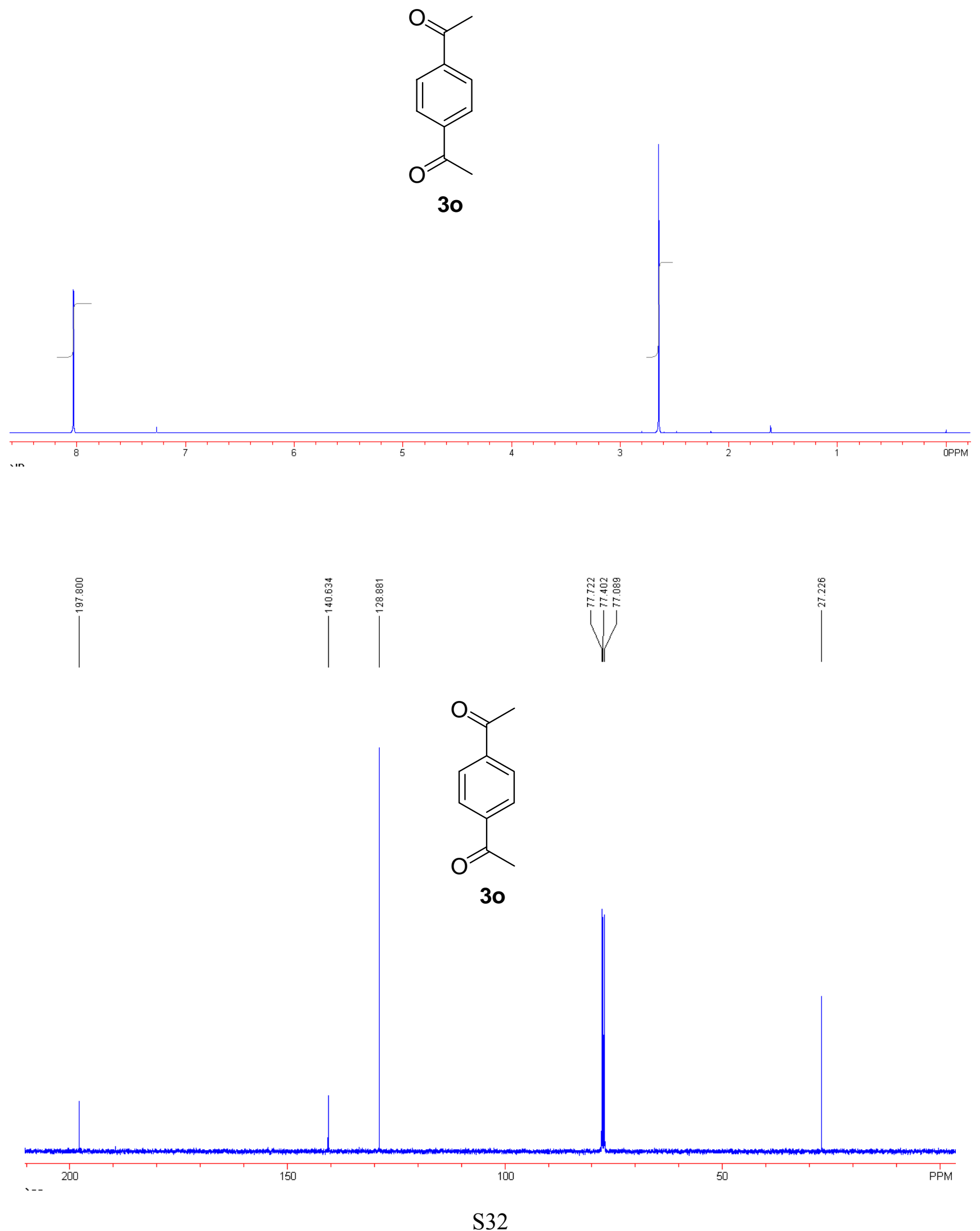
$\left.\right|^{\substack{n \\ n=0}}$
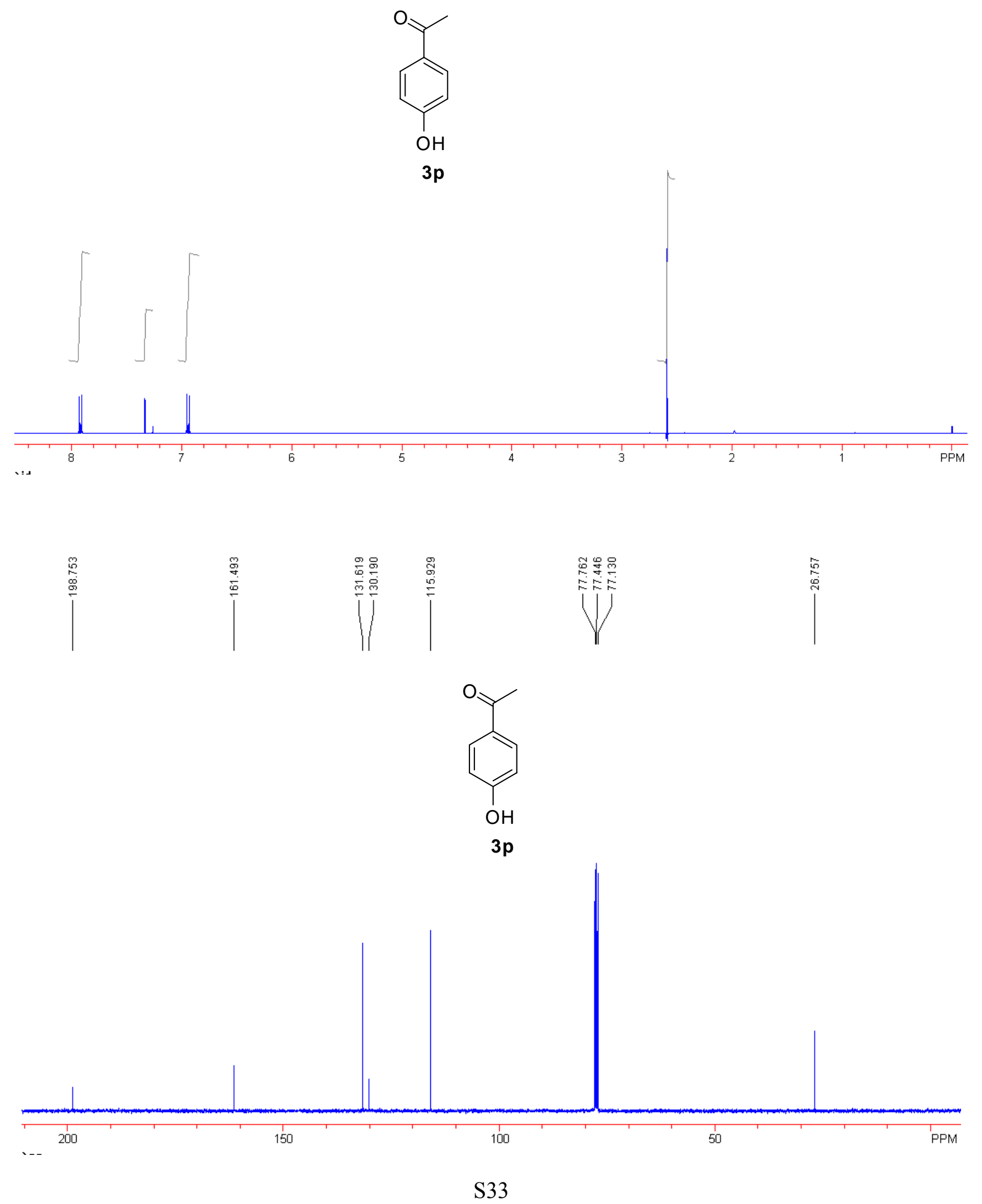
$\left.\right|^{\text {势 }}$
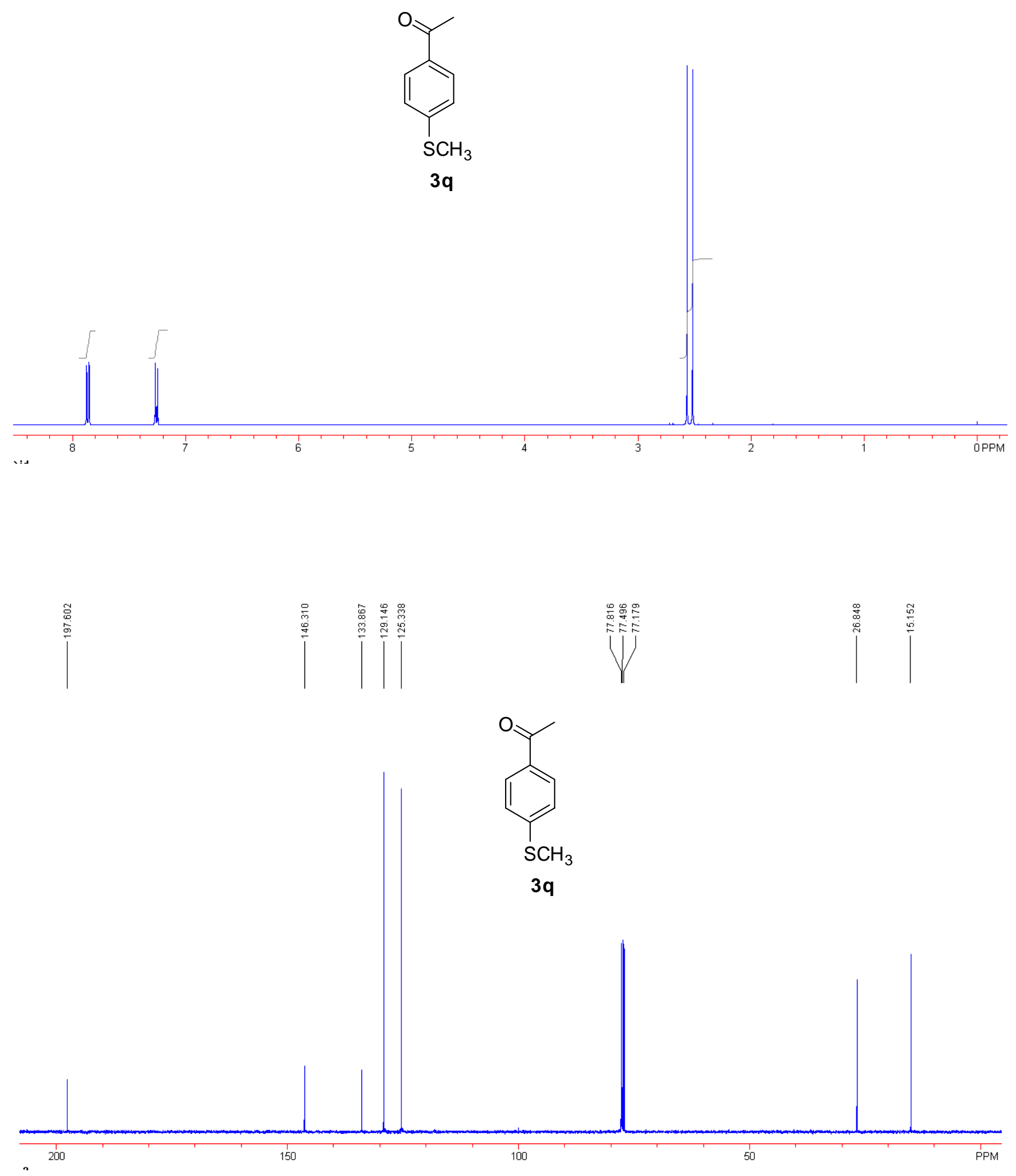
$\|^{1}$

$\mid$
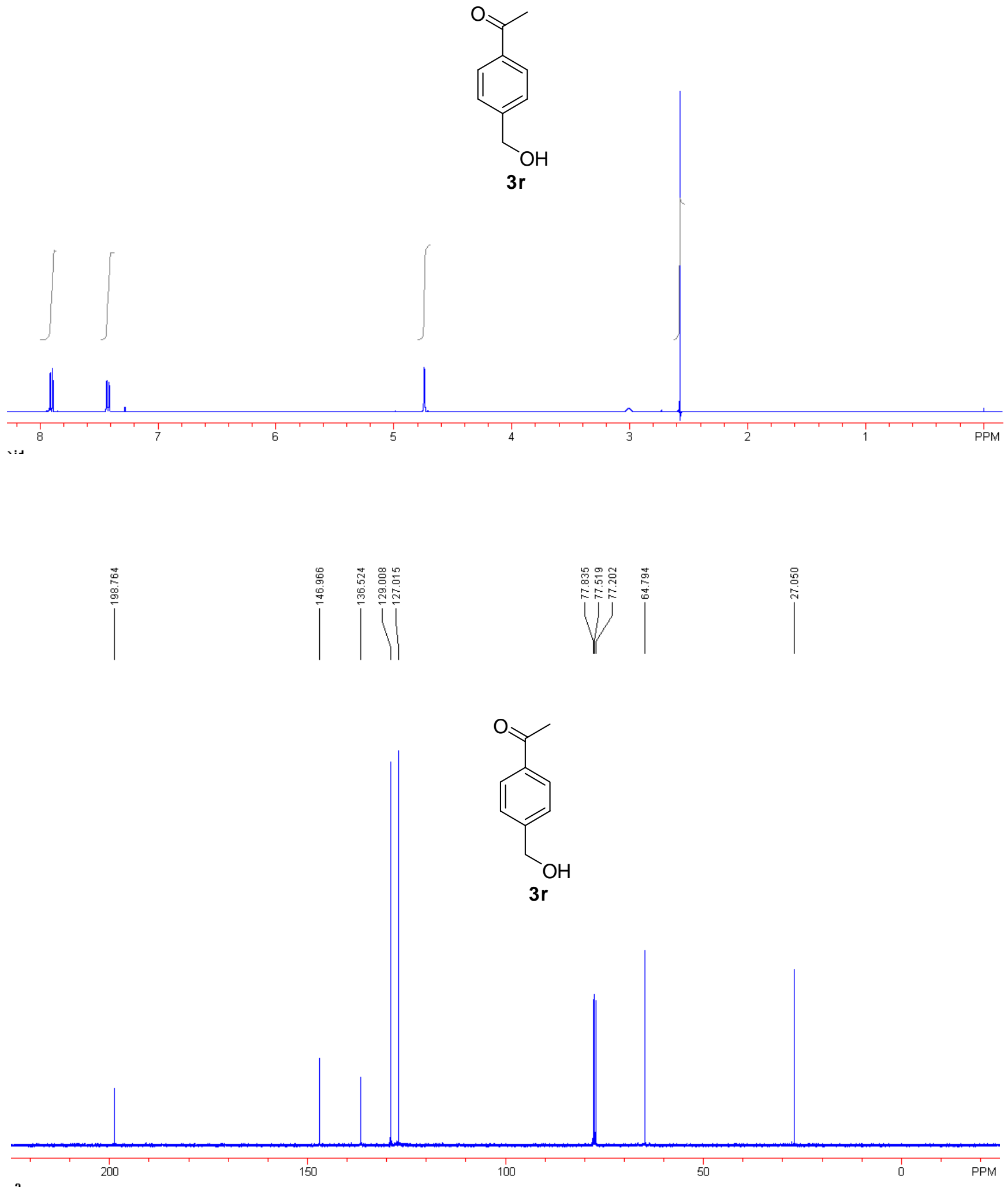

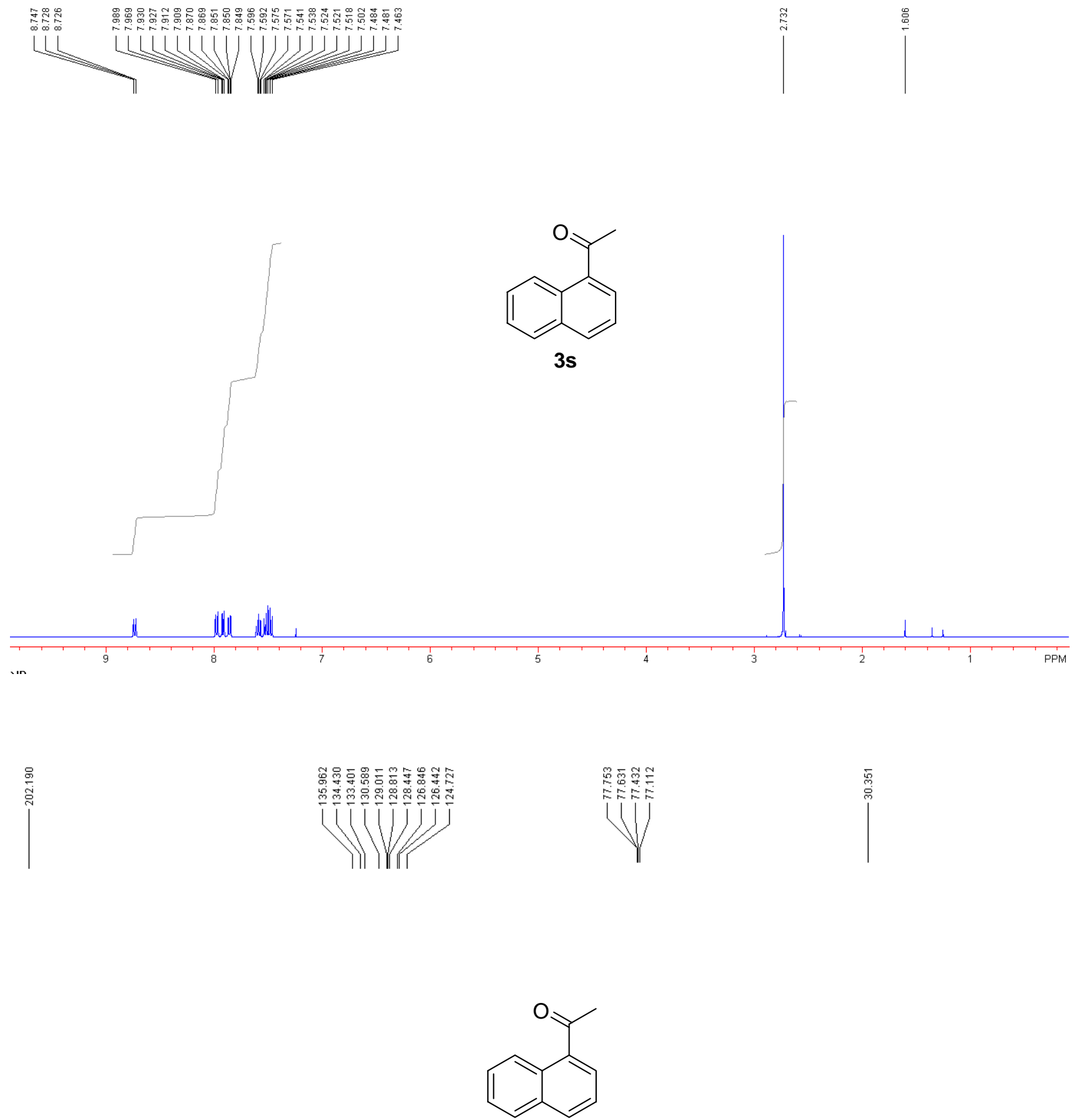

$3 s$

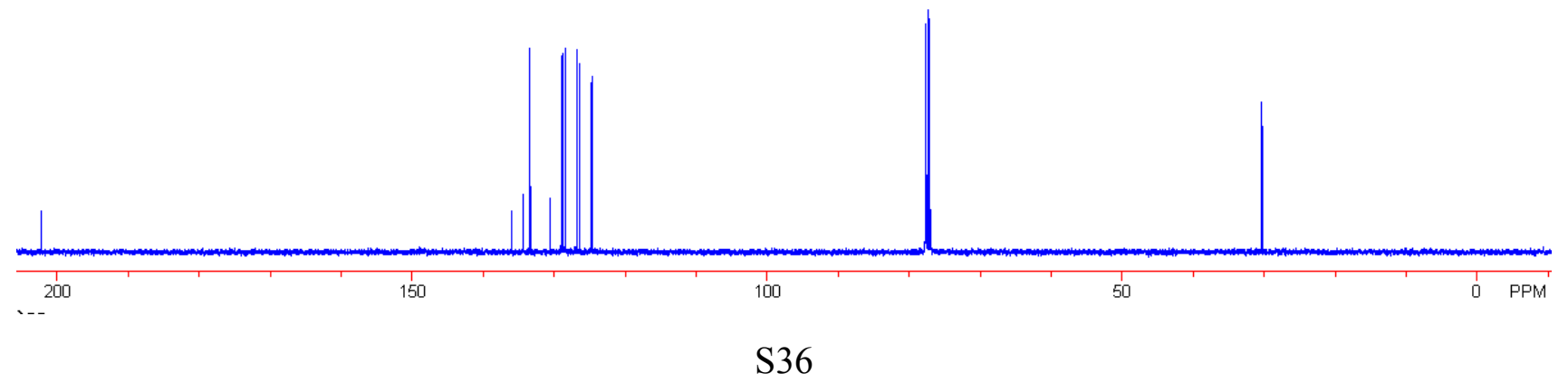



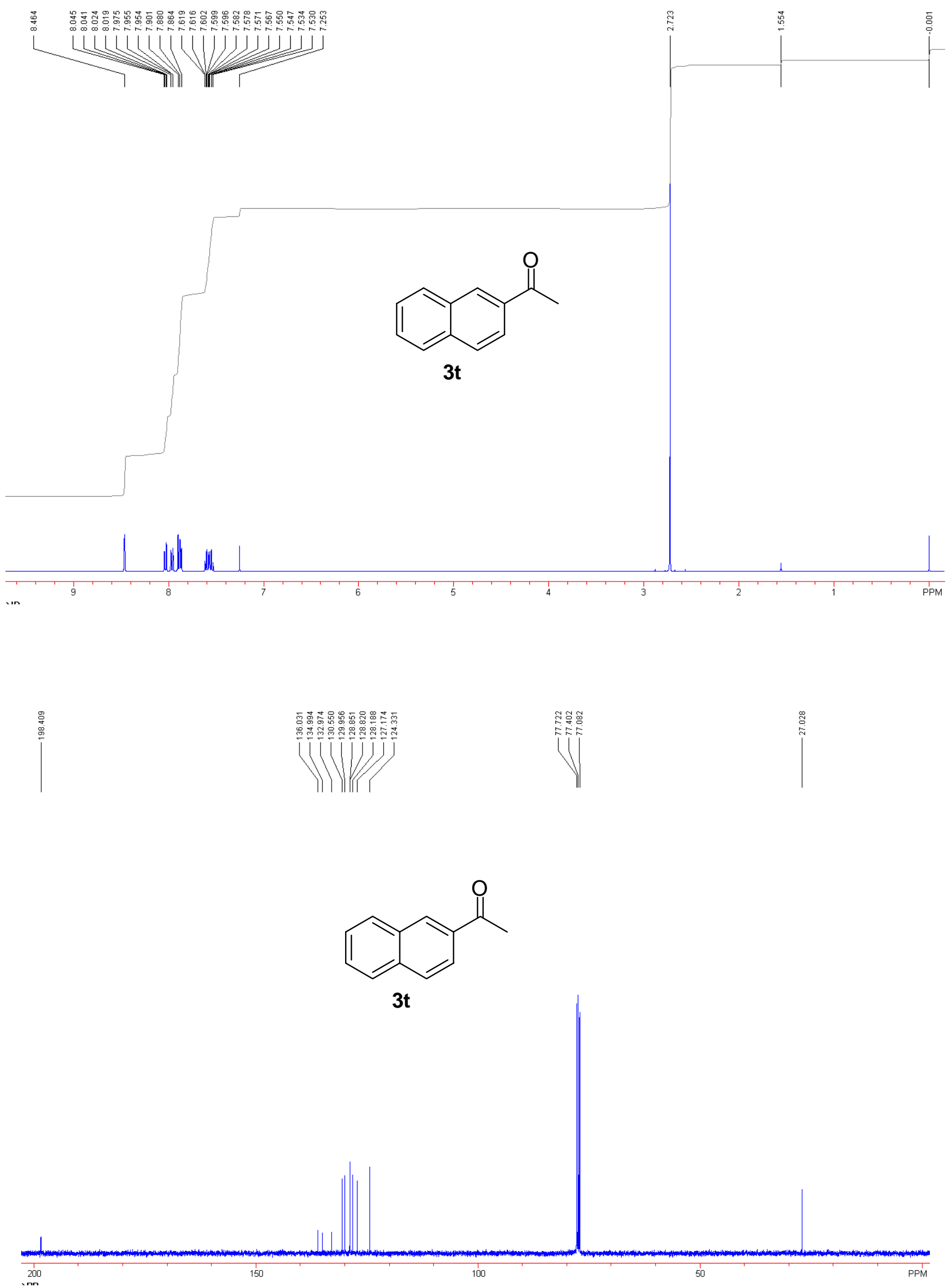
<smiles>CCC(=O)c1ccccc1</smiles>

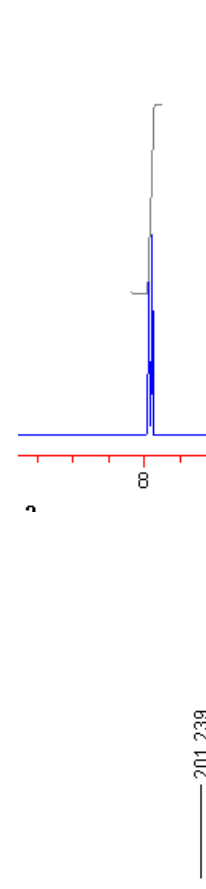

$3 u$
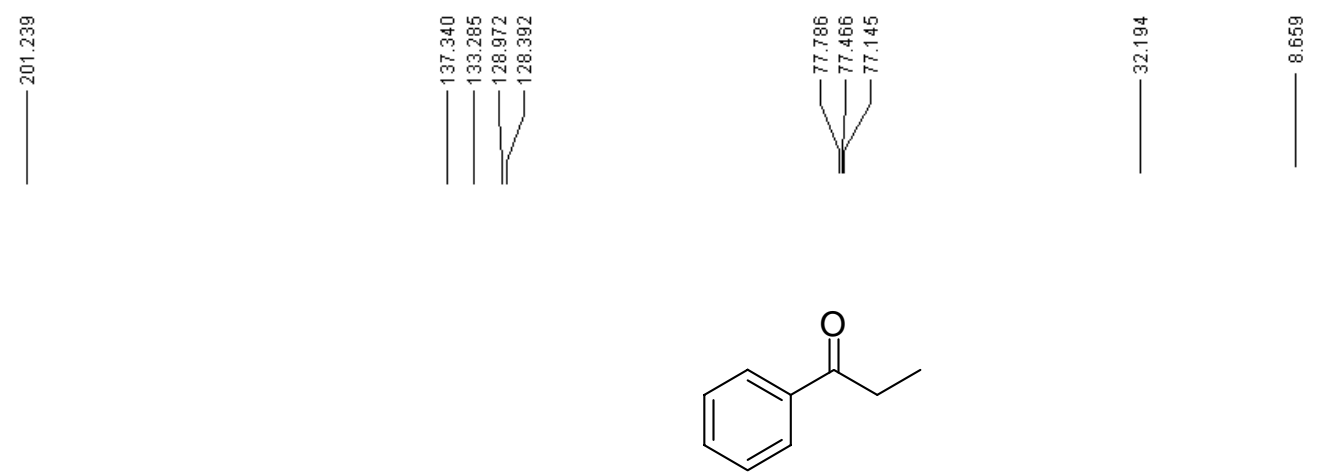

$3 u$

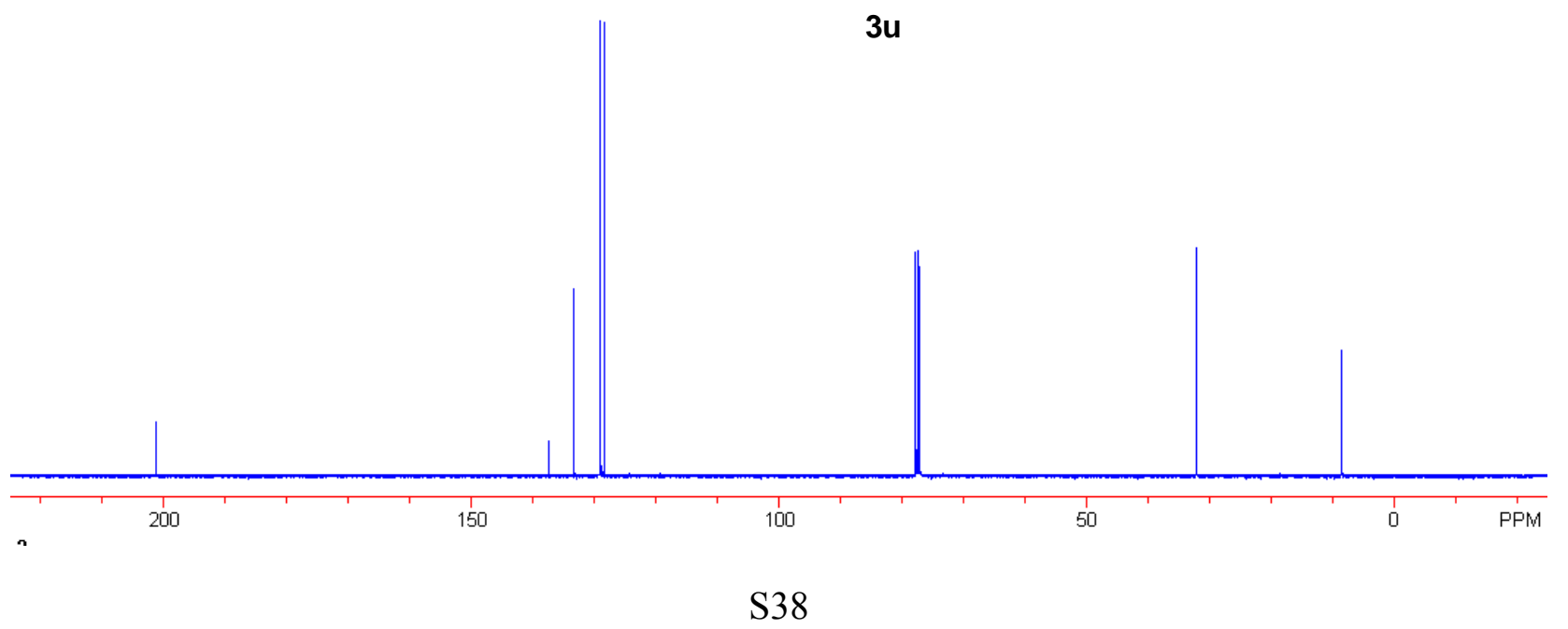




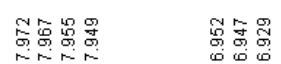

U)

VI

$\left.\right|_{1} ^{\substack{n \\ \infty}}$

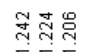

Vij<smiles>CCC(=O)c1ccc(OC)cc1</smiles>
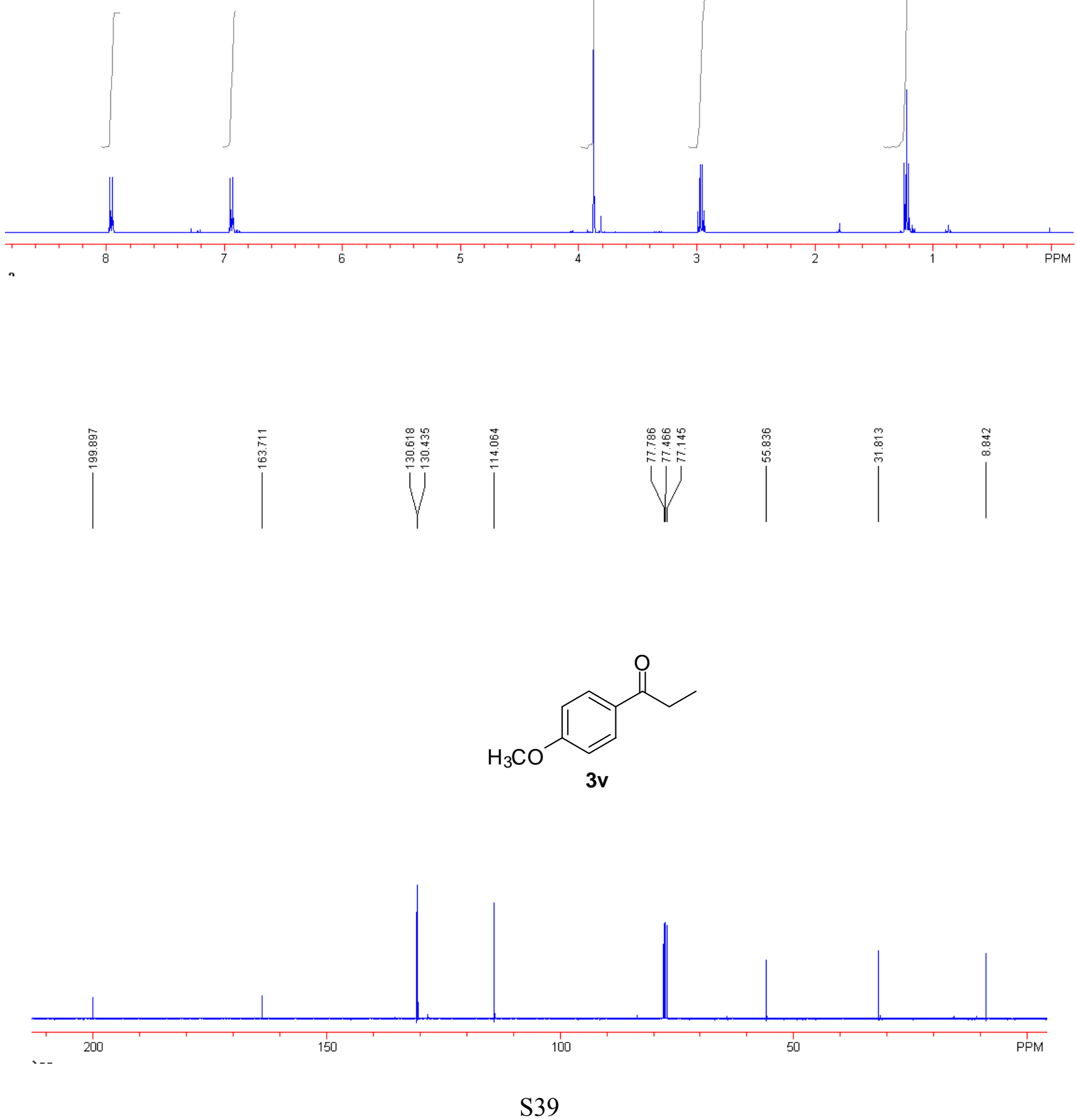


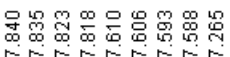

iUNij

兽要骂哭

U)

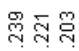

W
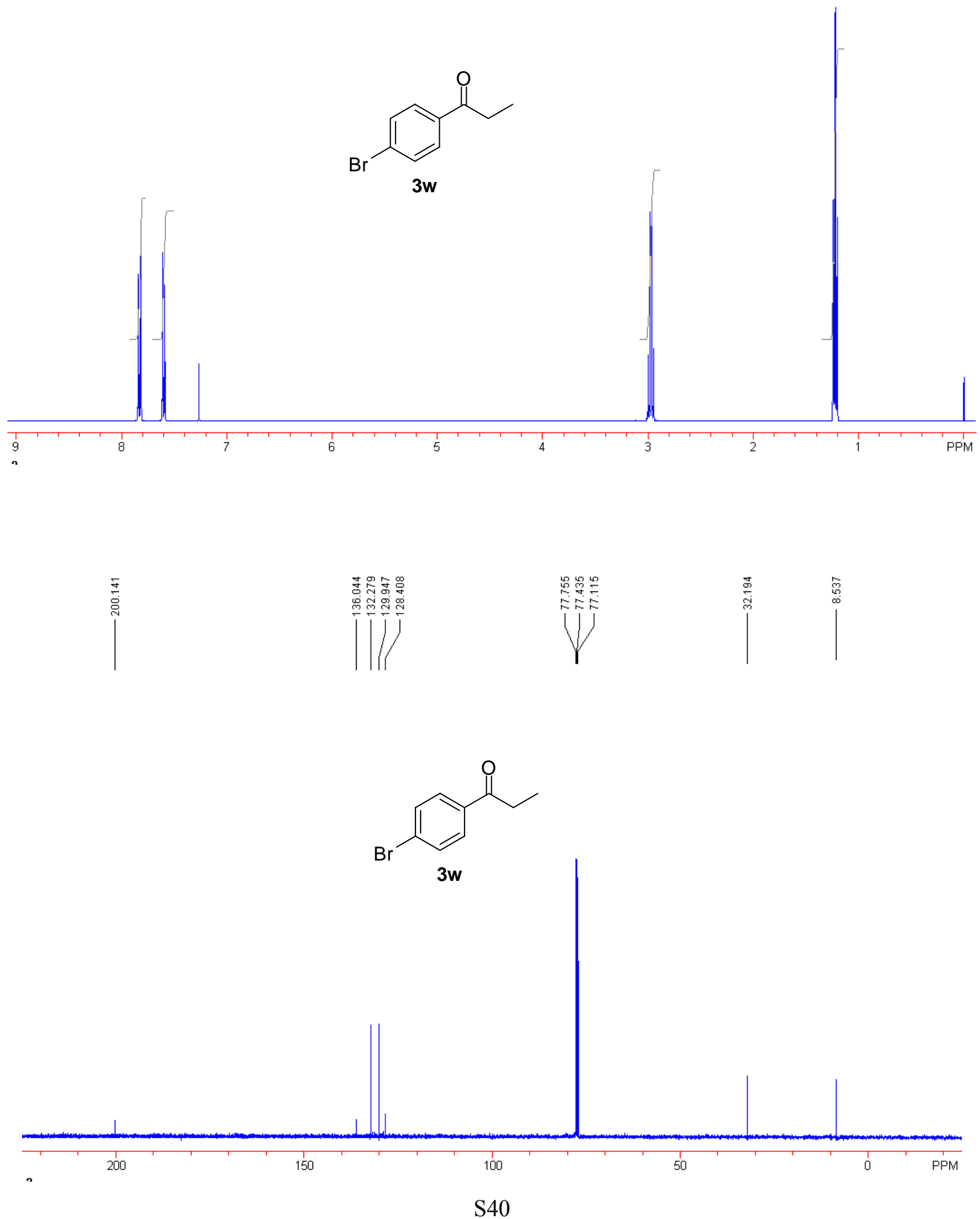

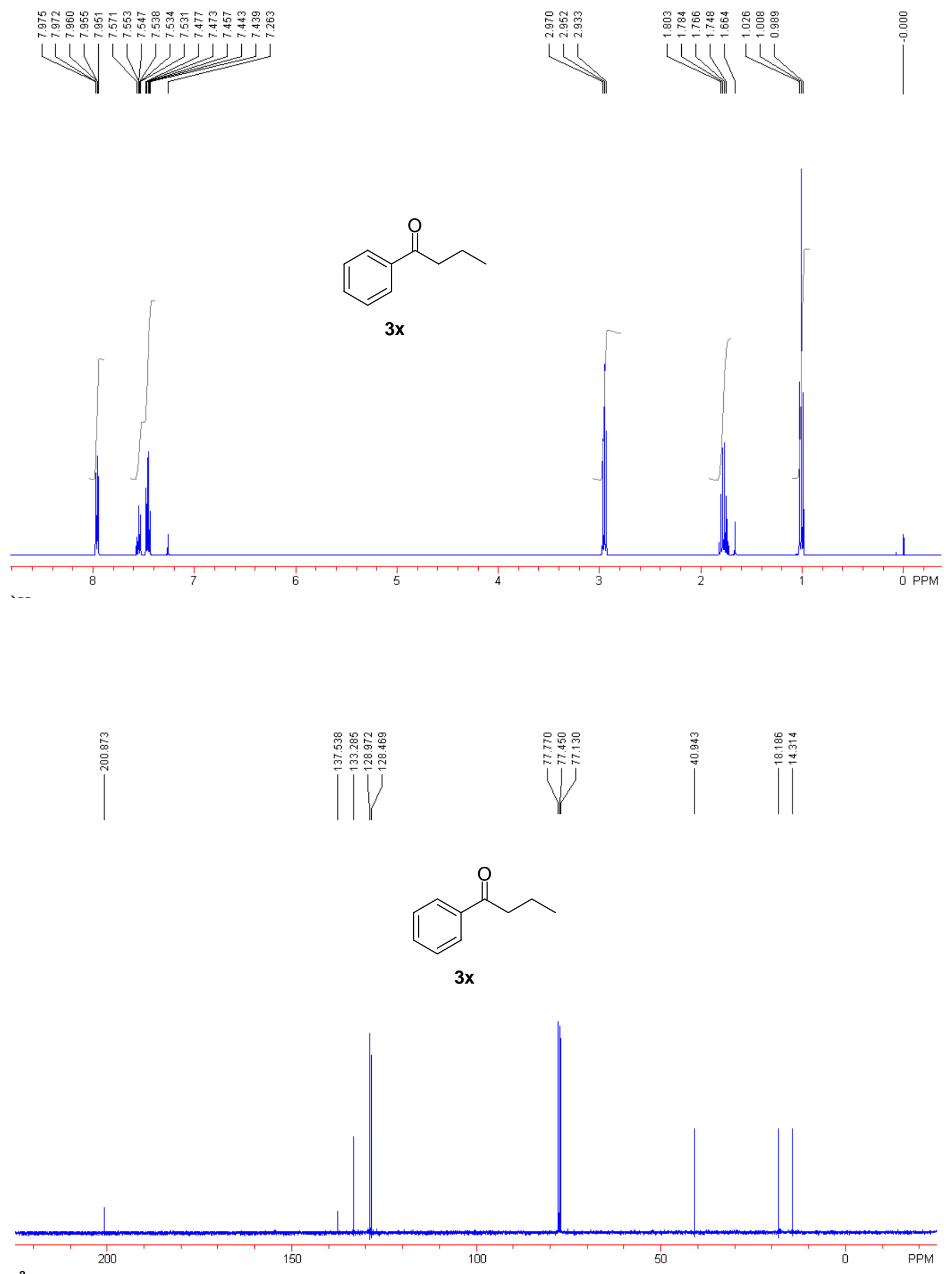

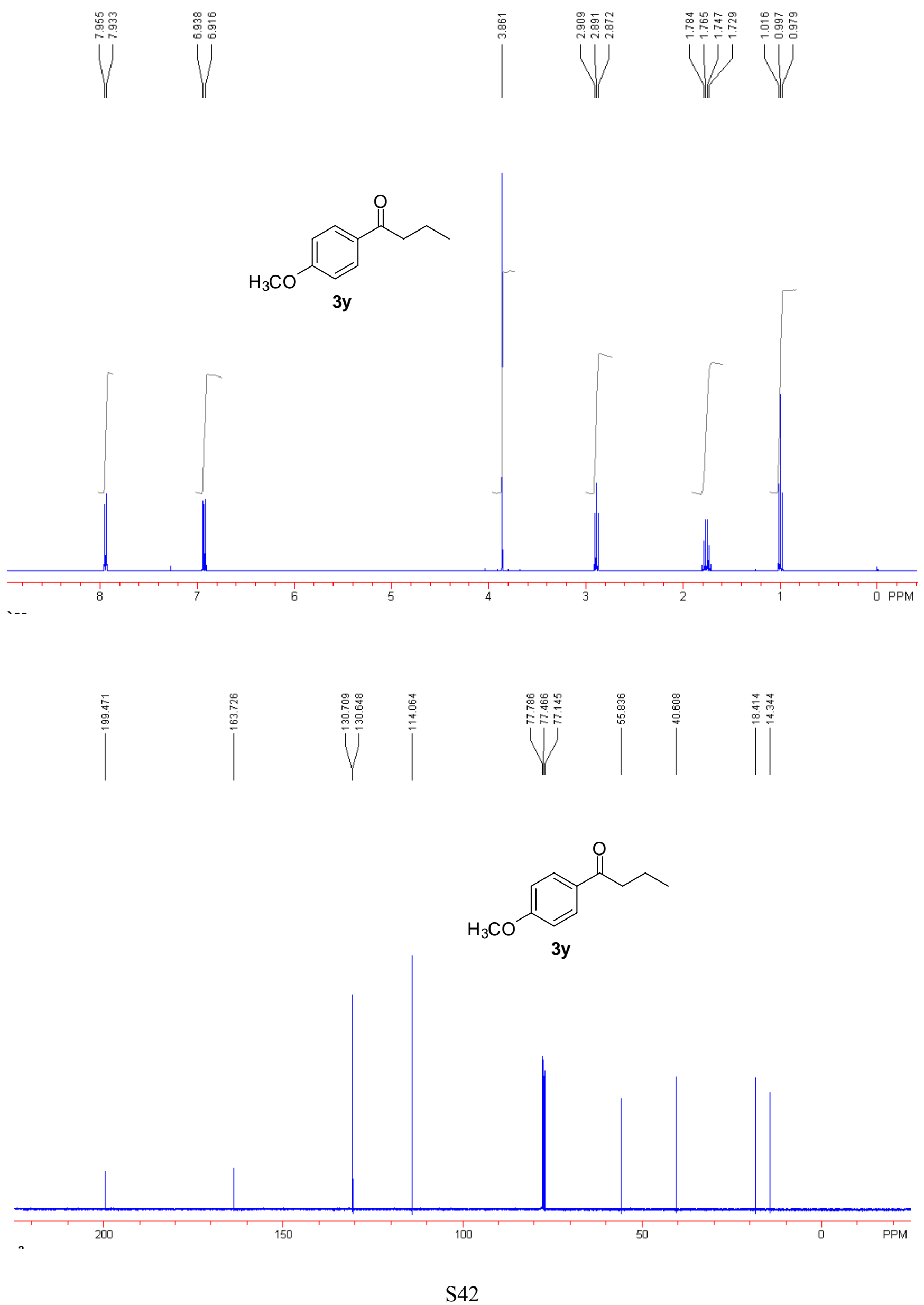


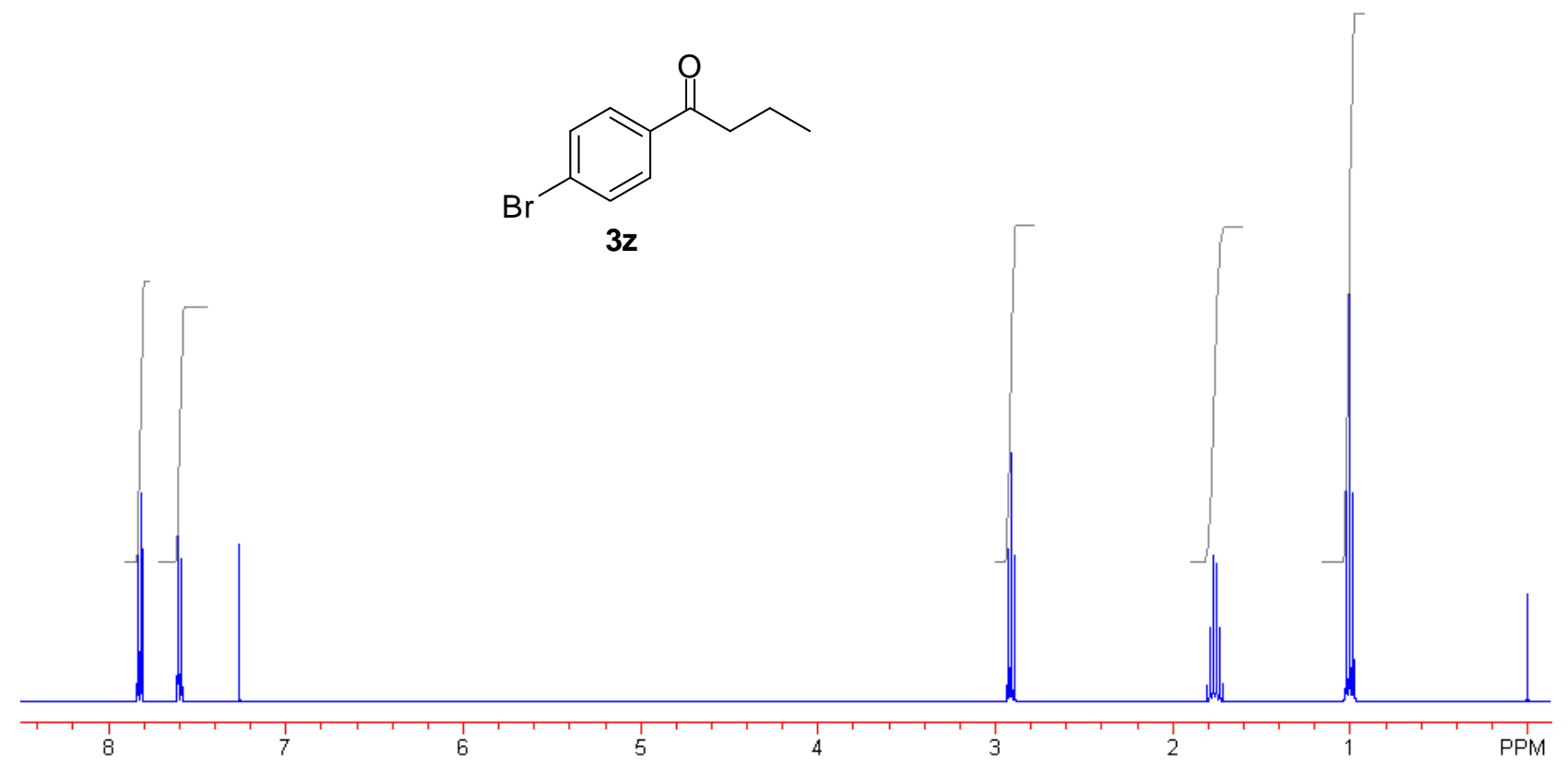
--
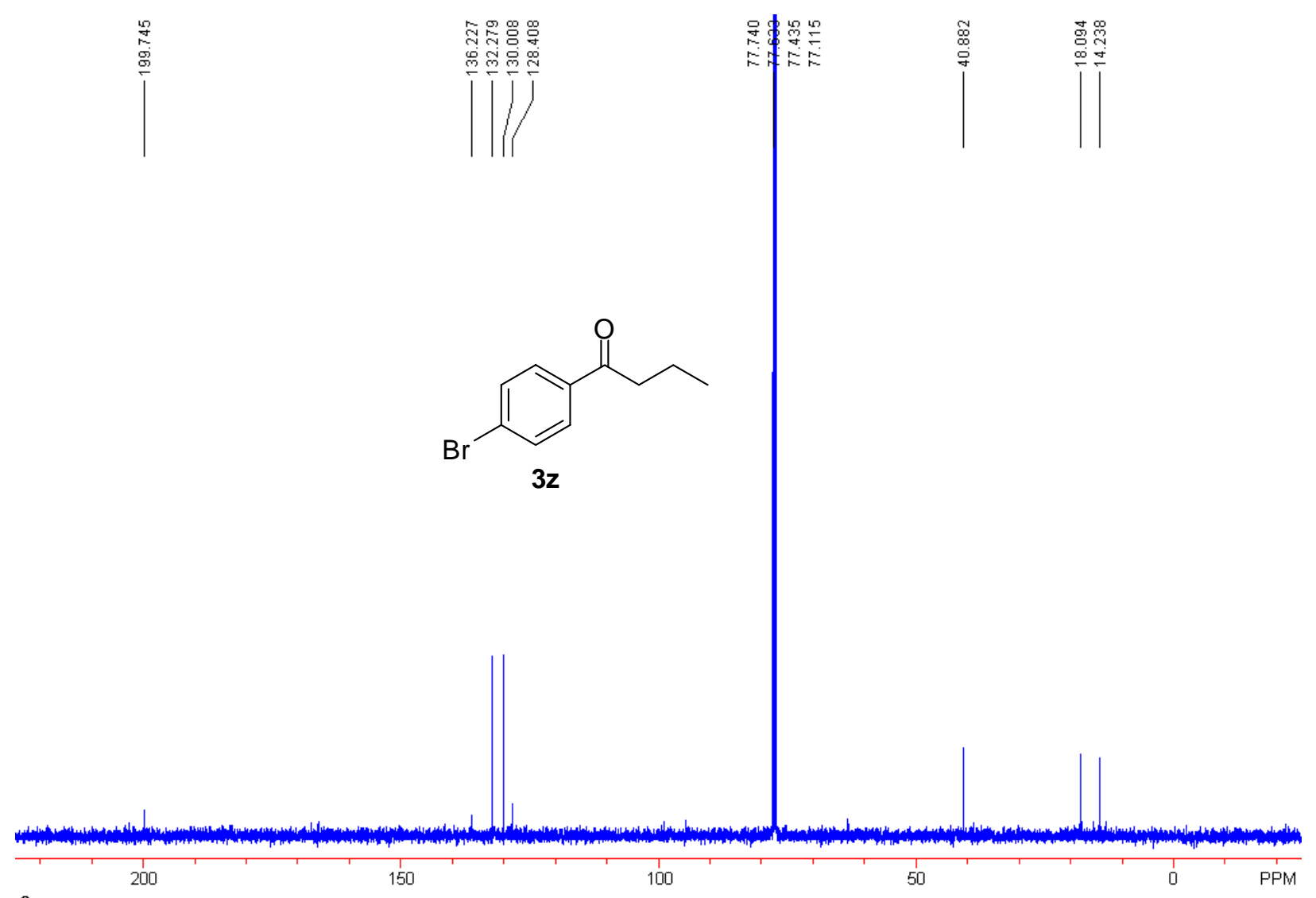

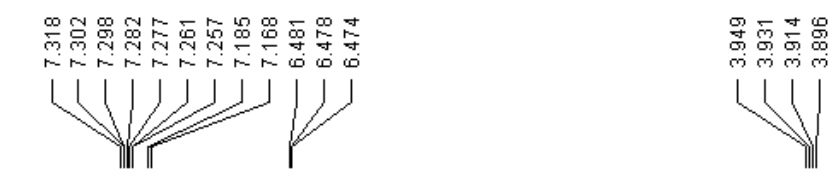

!
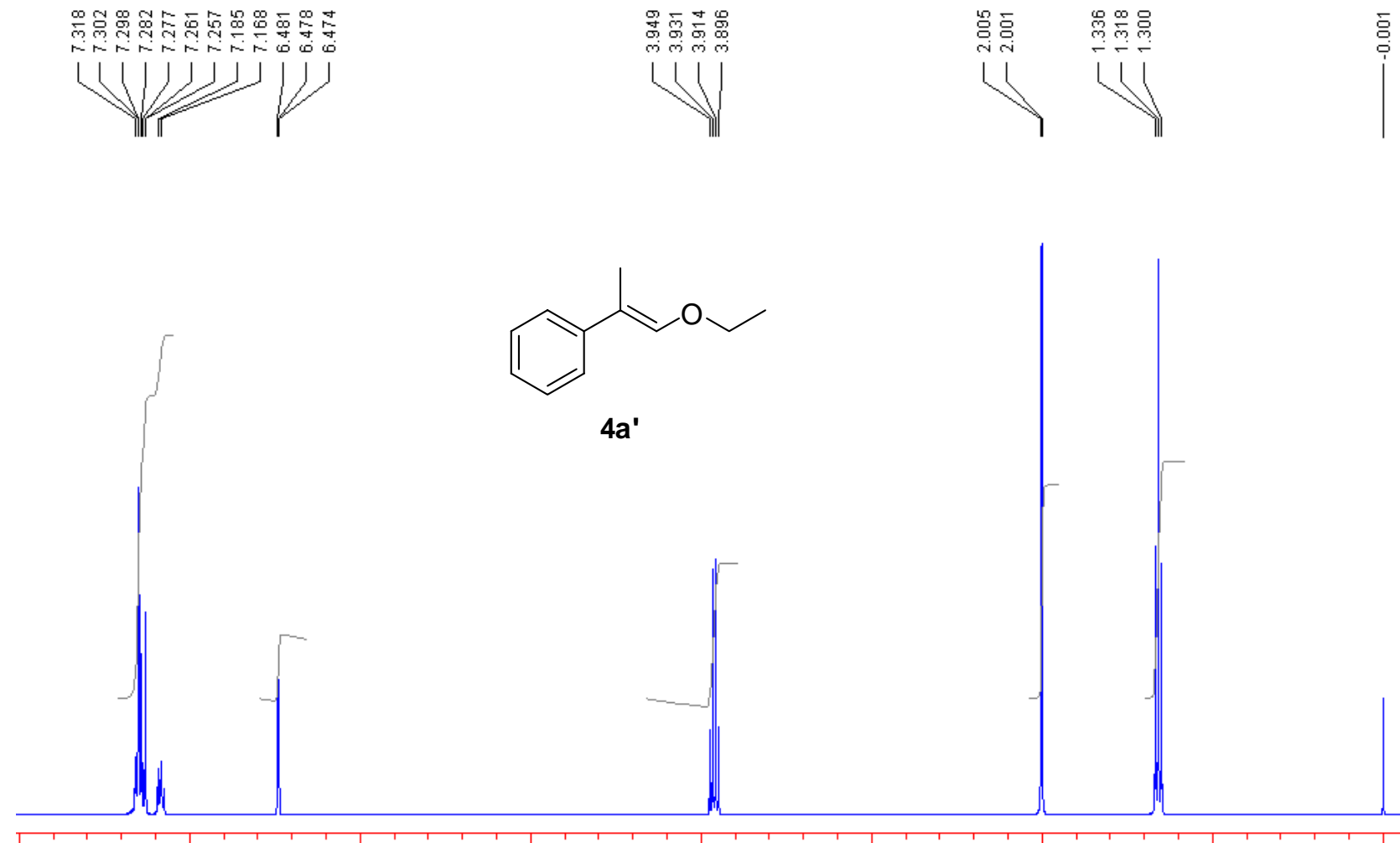

$4 a^{\prime}$
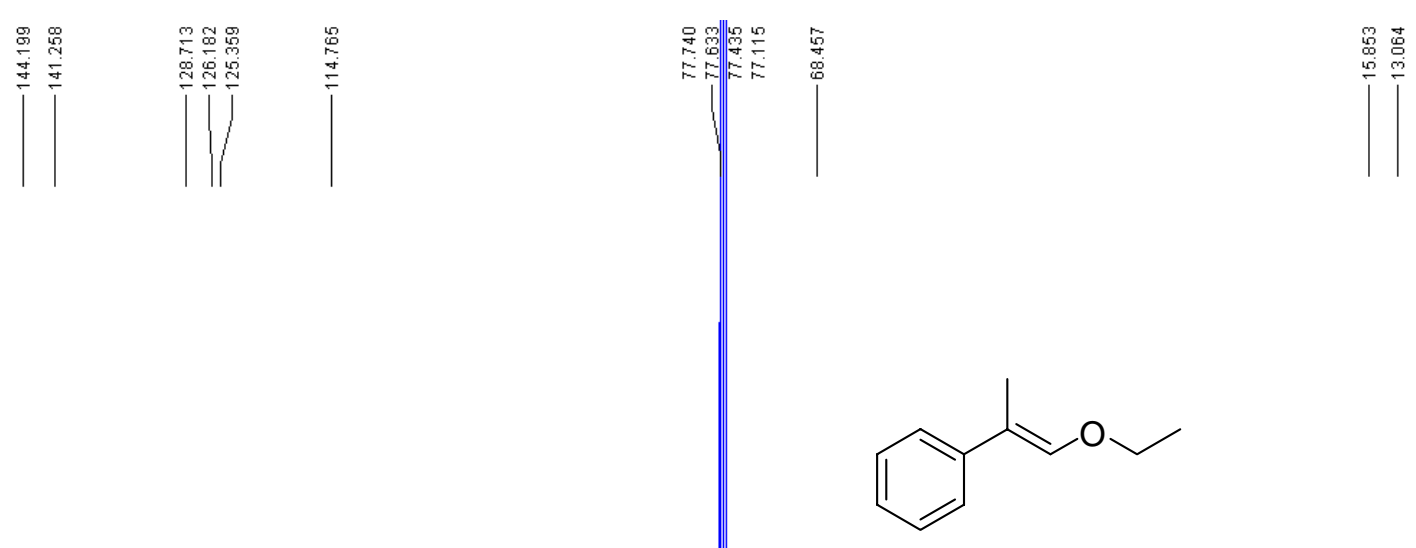

$4 a^{\prime}$

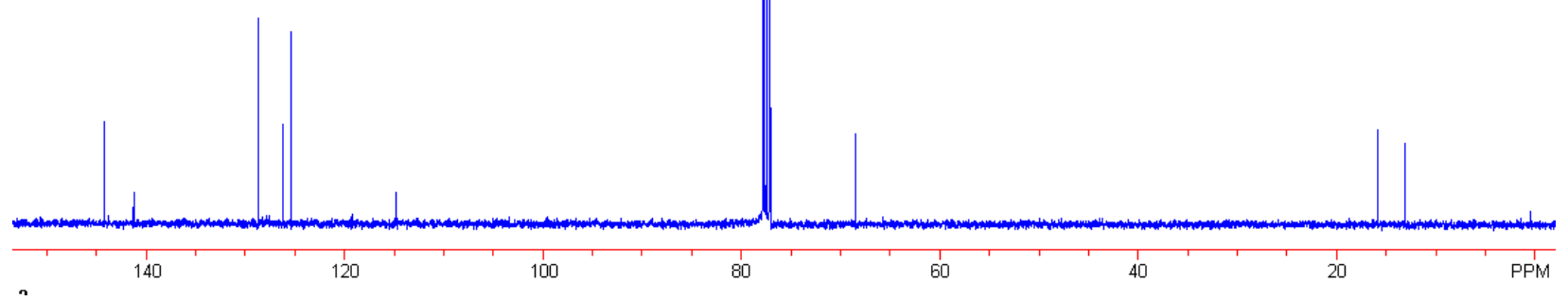




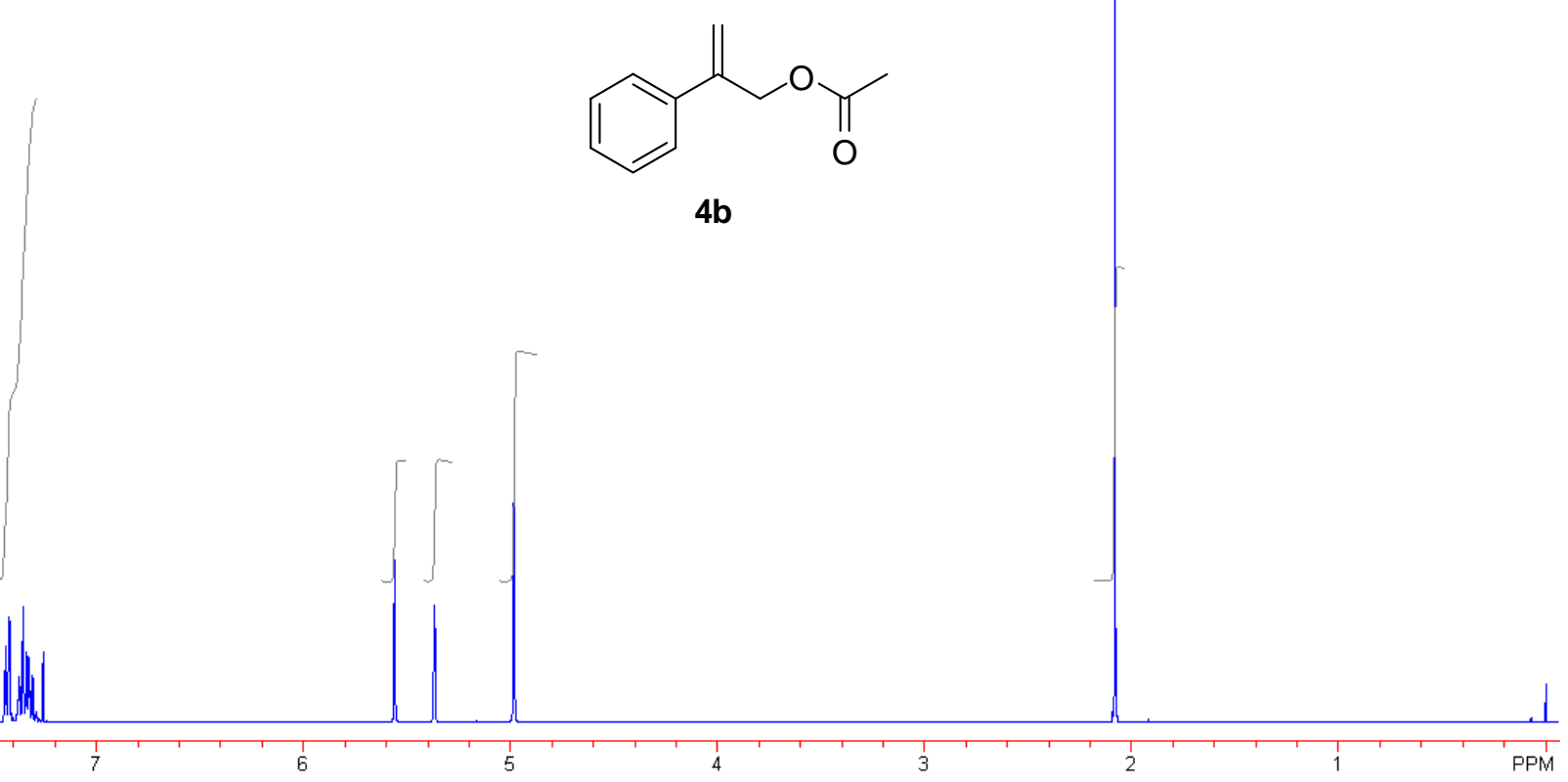

$4 b$
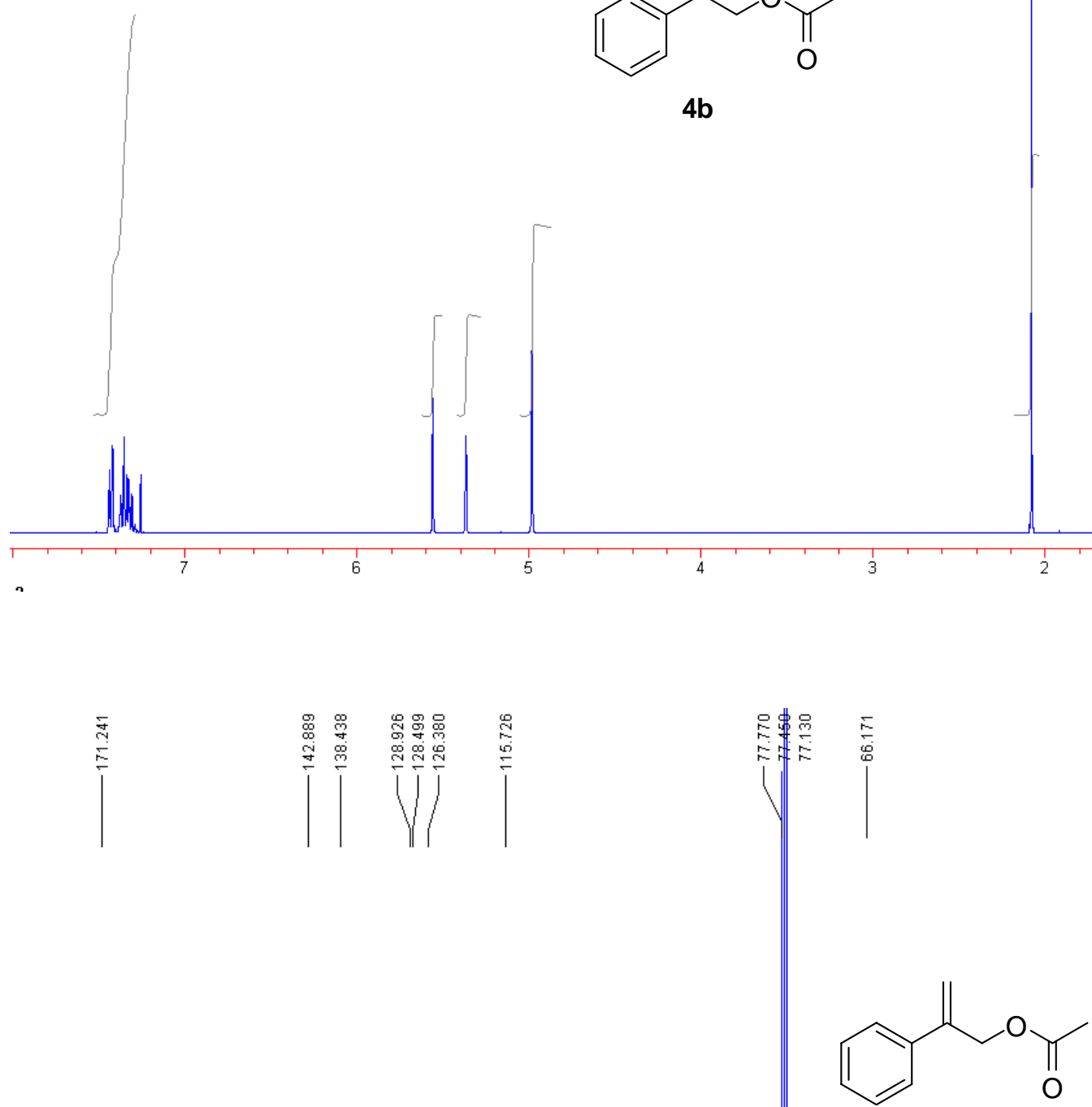

$4 b$

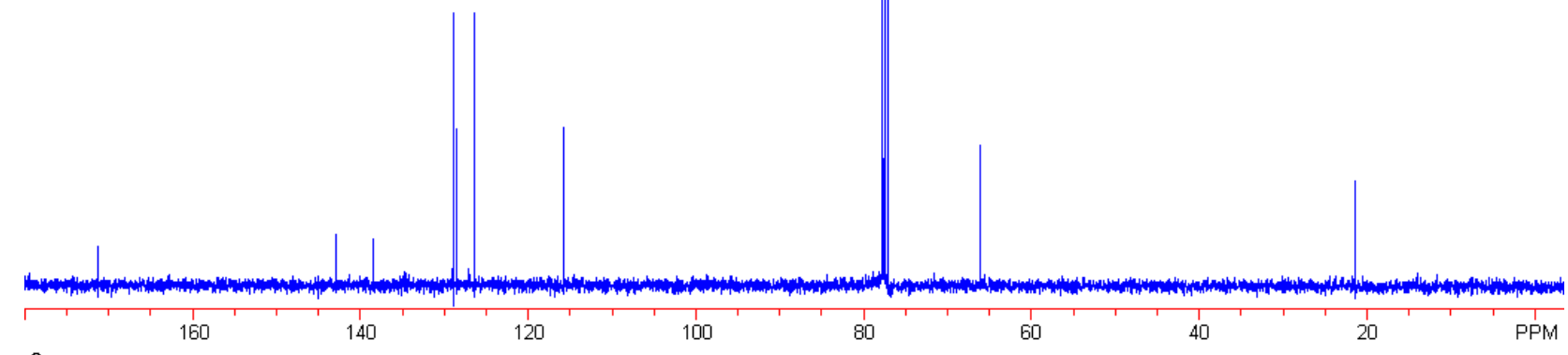



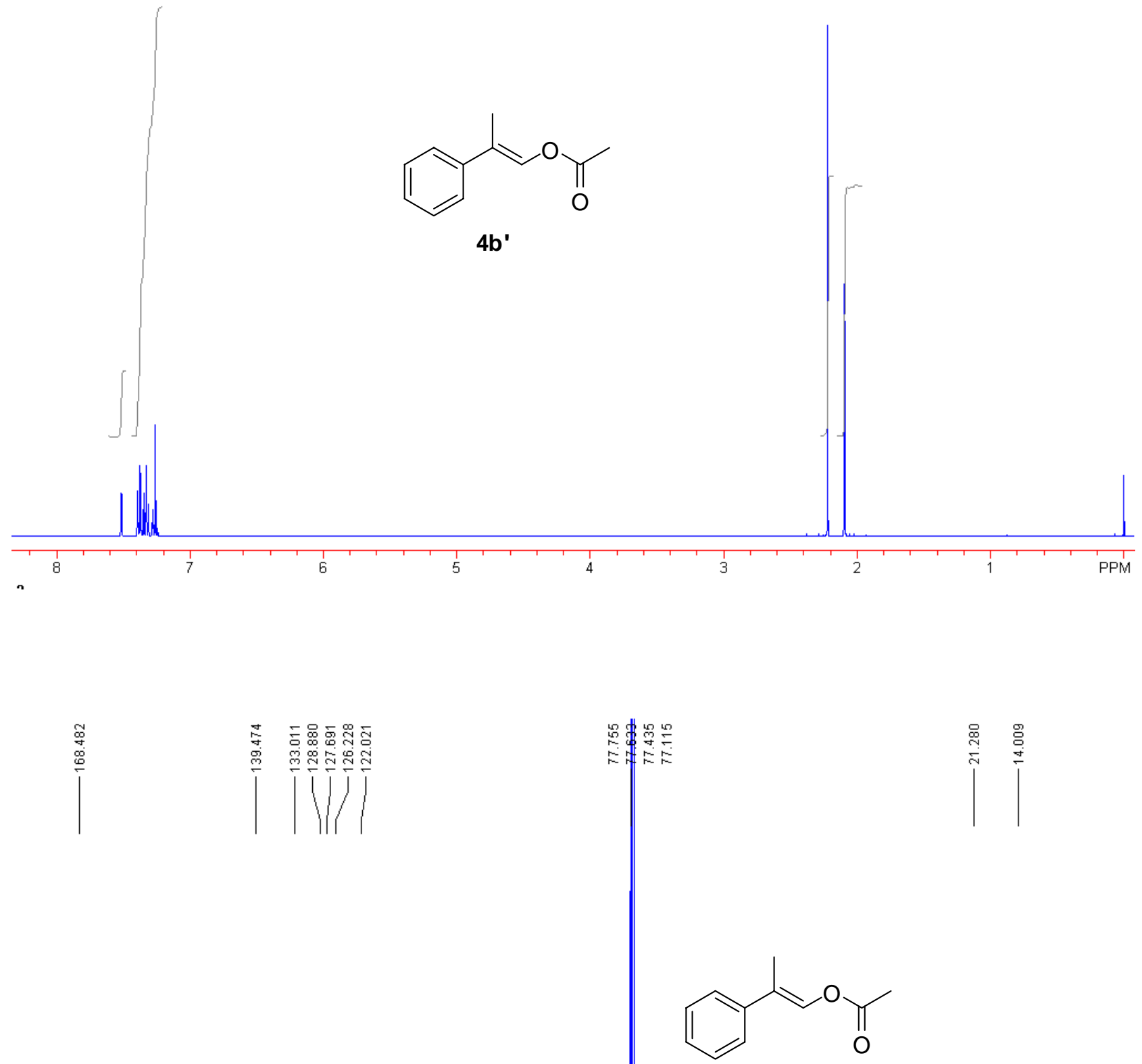

$4 b^{\prime}$

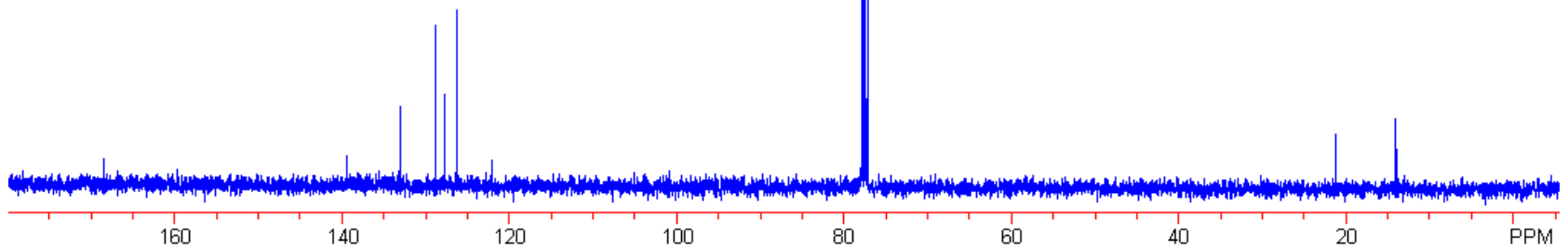



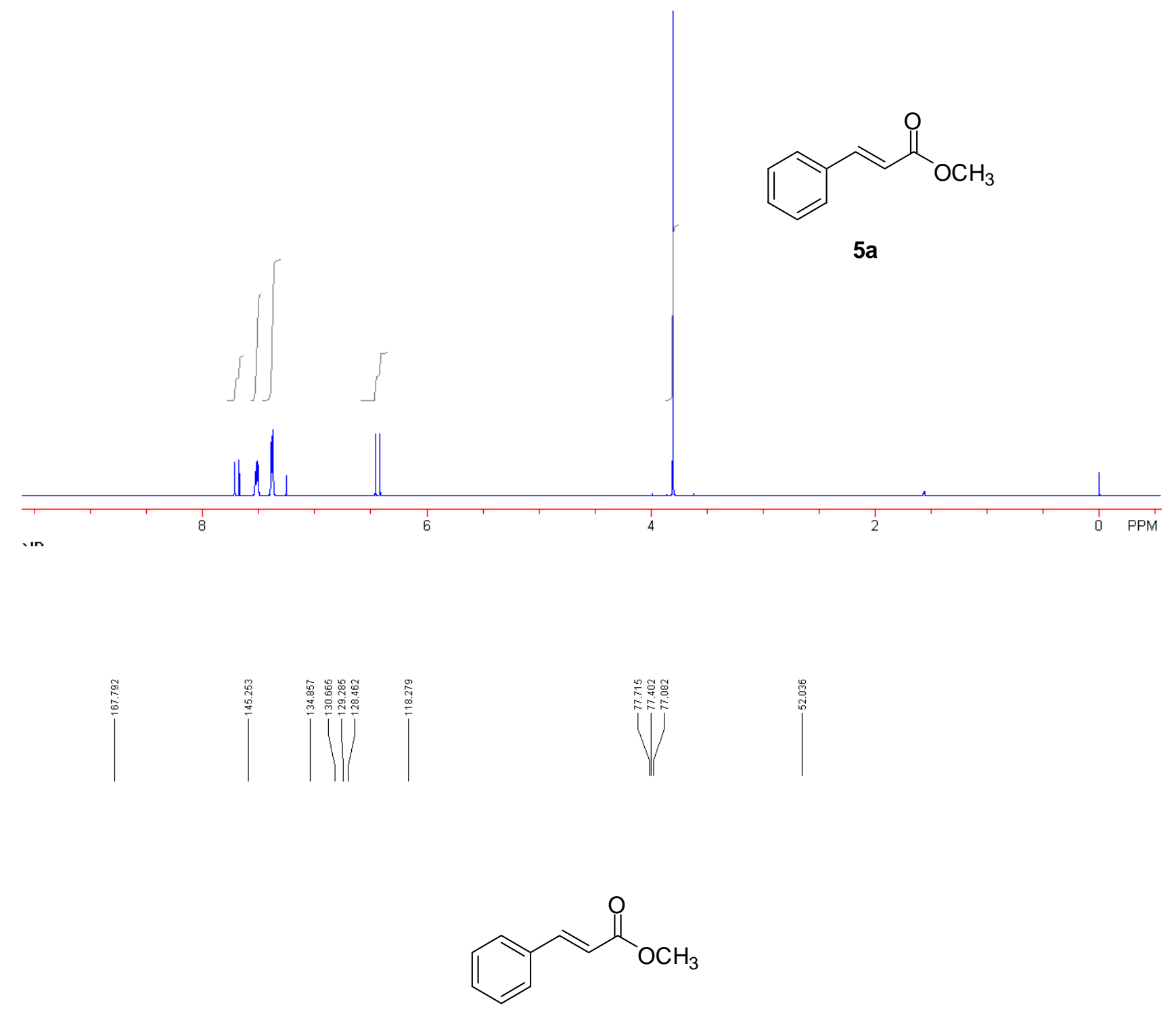

$5 a$

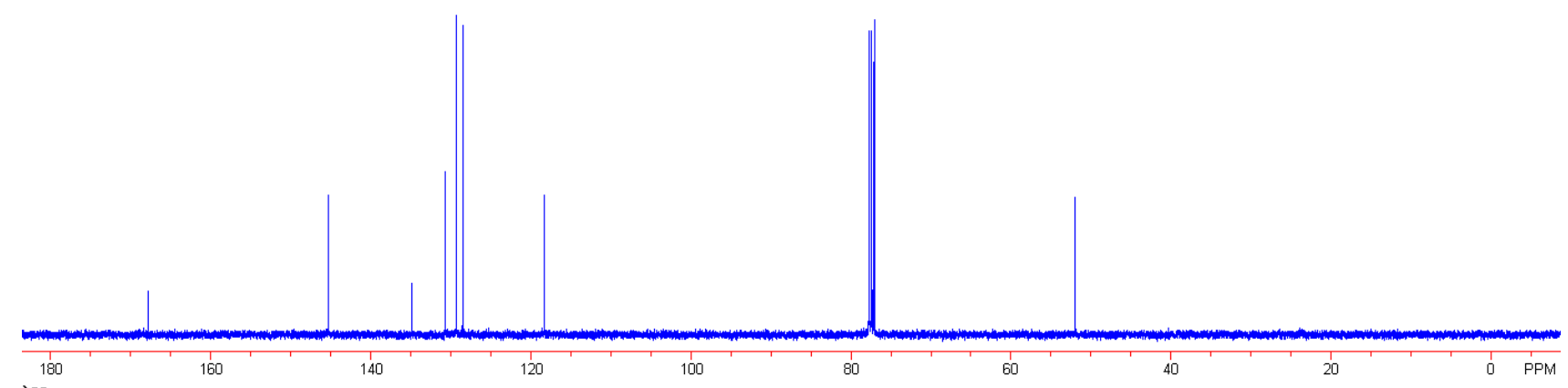




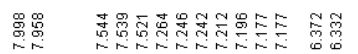

u
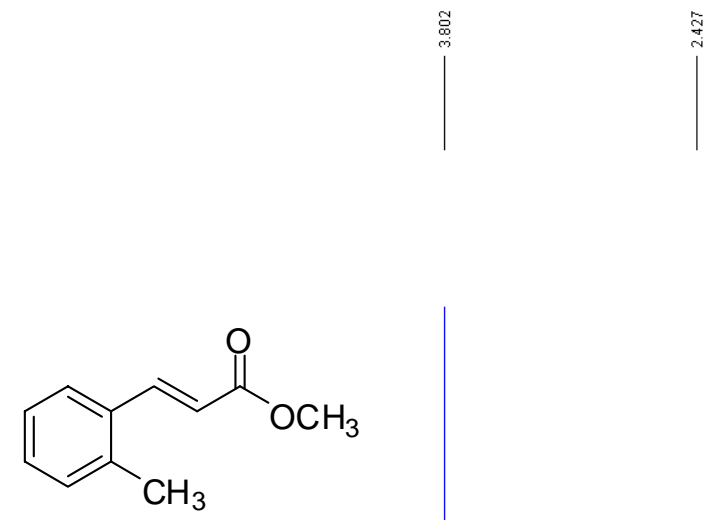

$5 b$

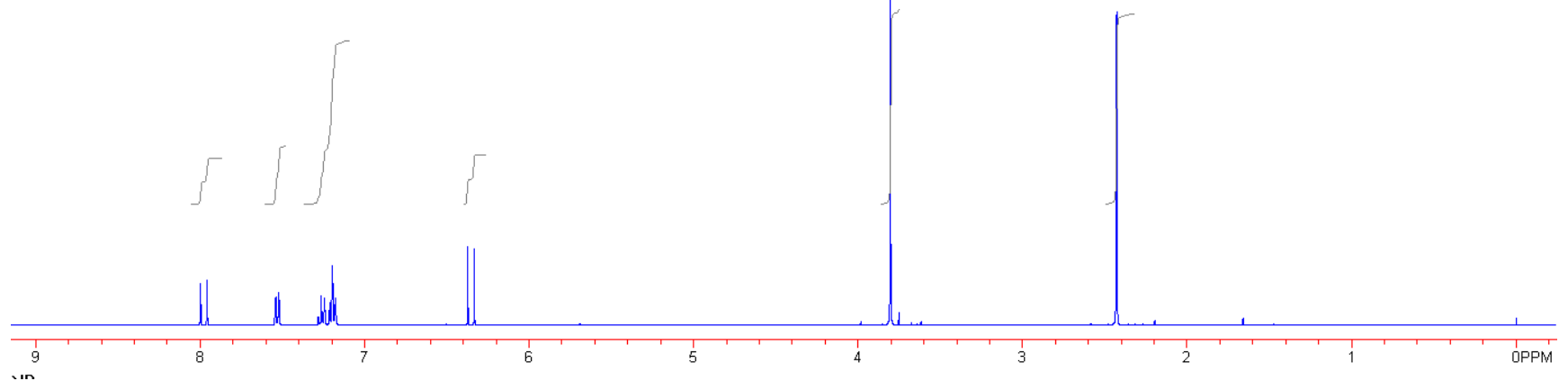<smiles>COC(=O)/C=C/c1ccccc1C</smiles>

5b

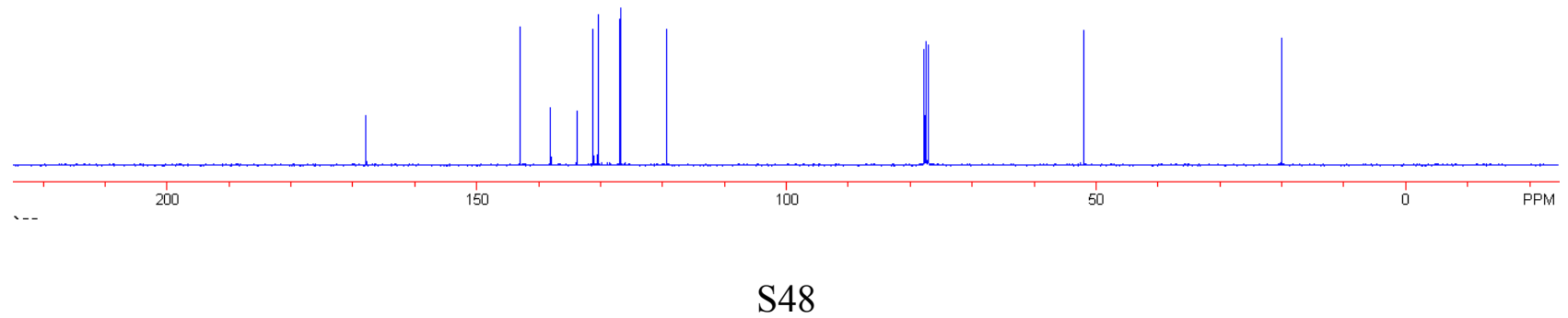



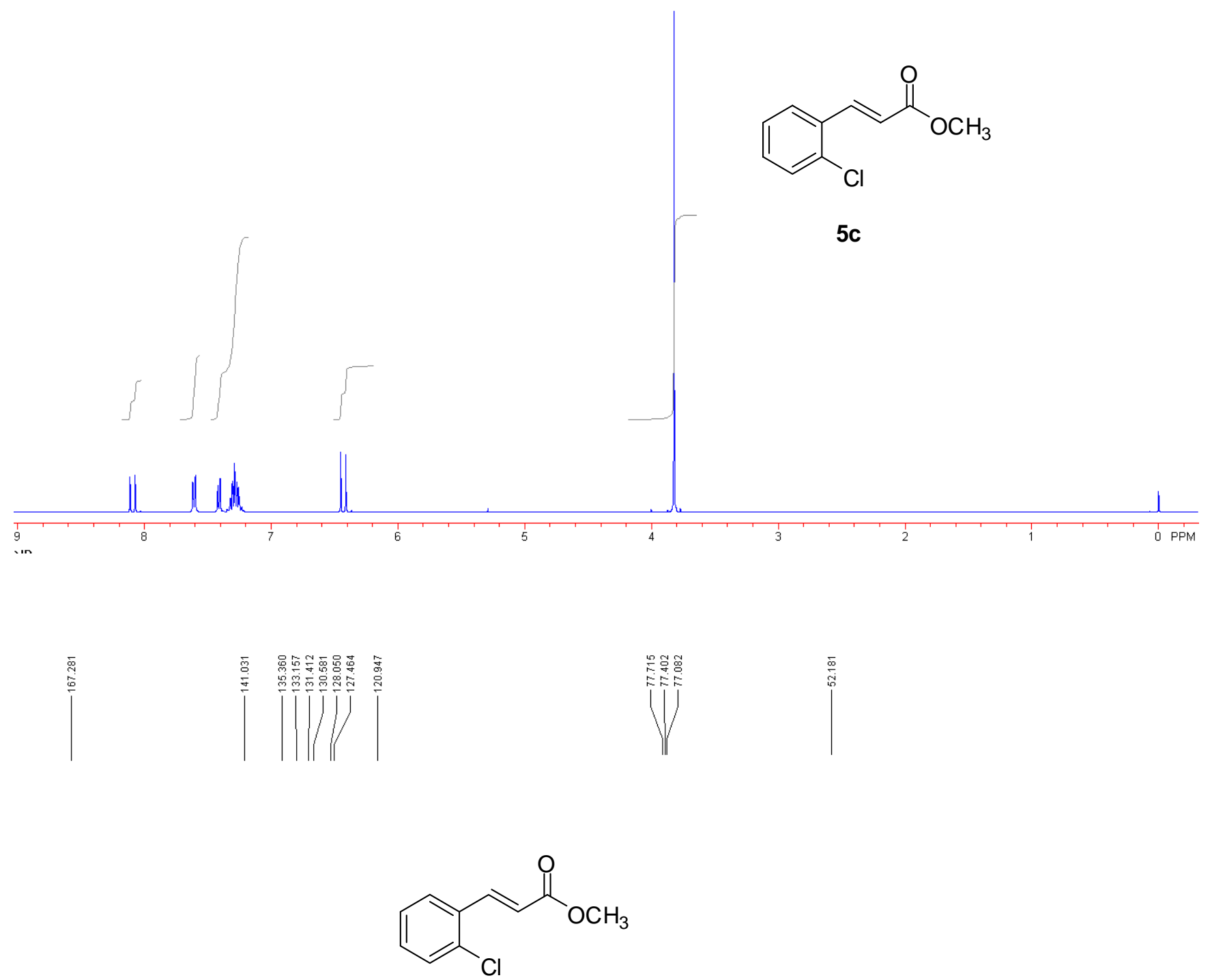

5c

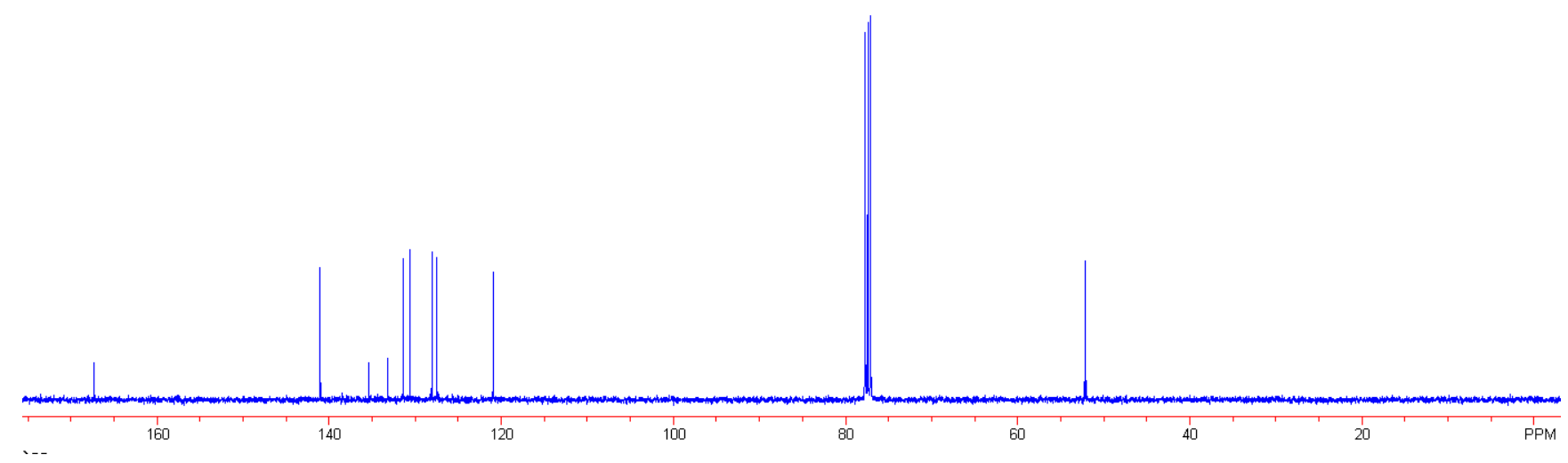



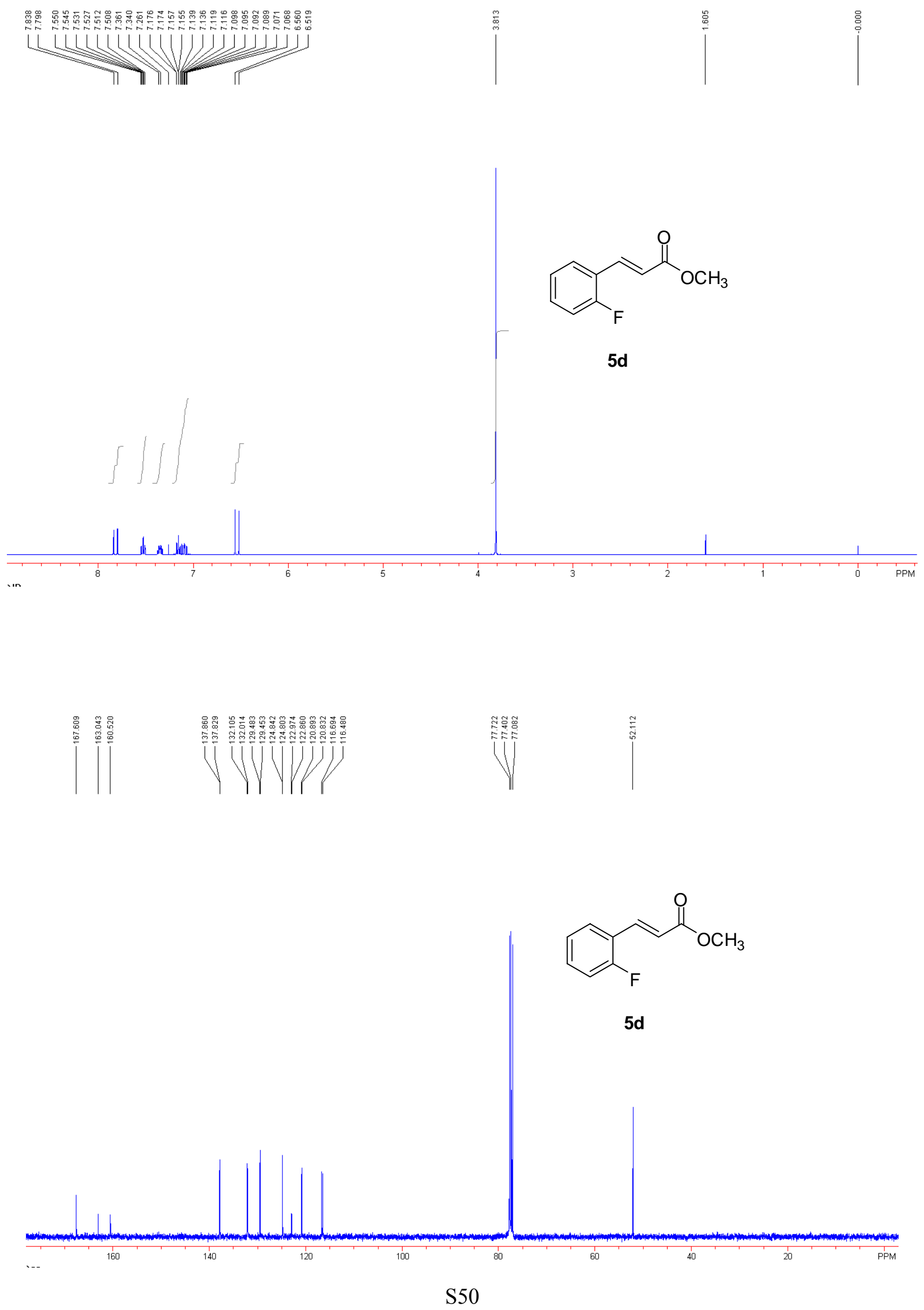


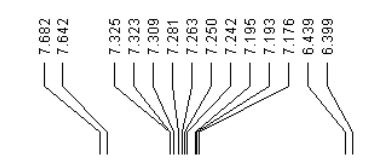

i|
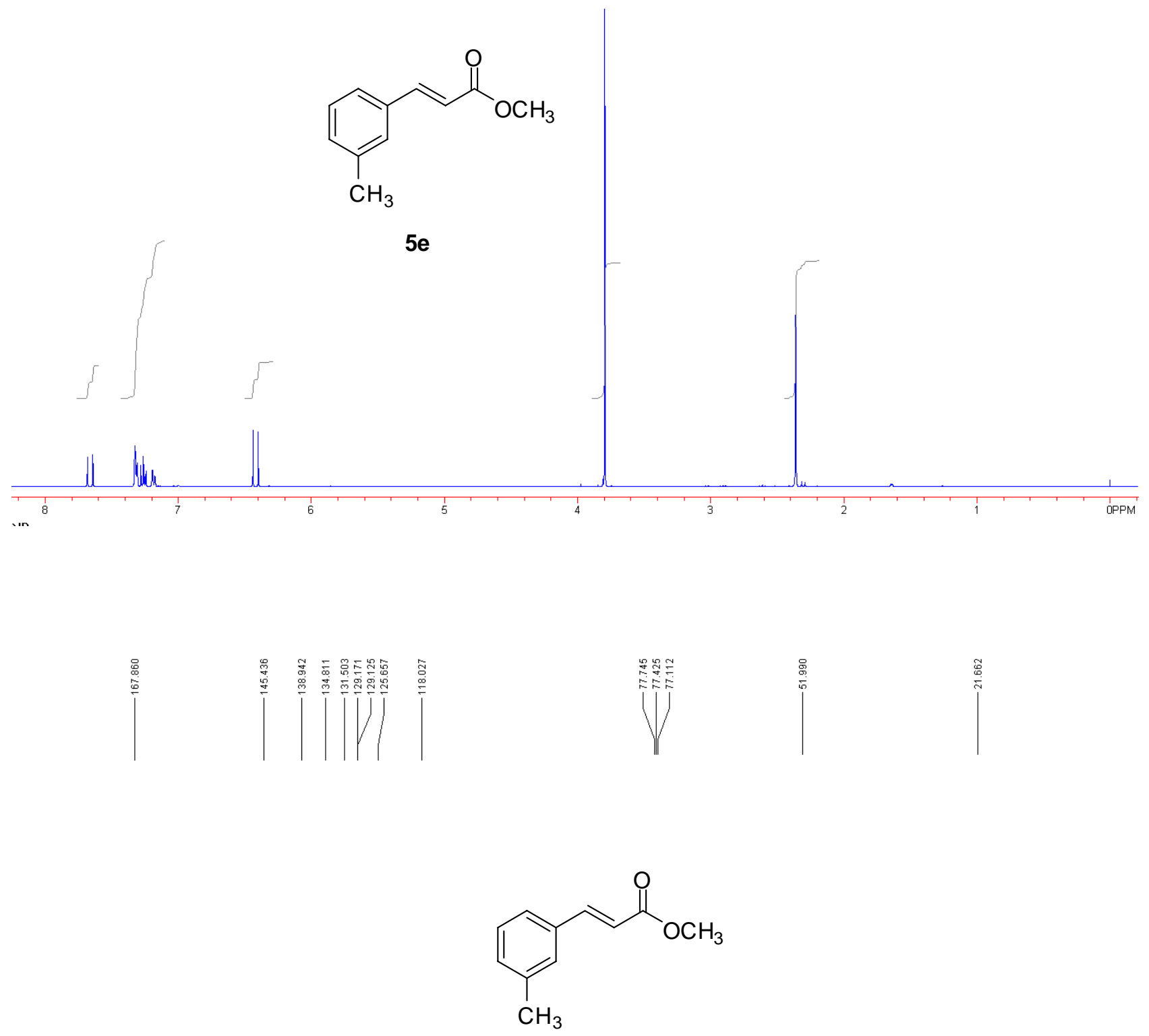

$5 e$

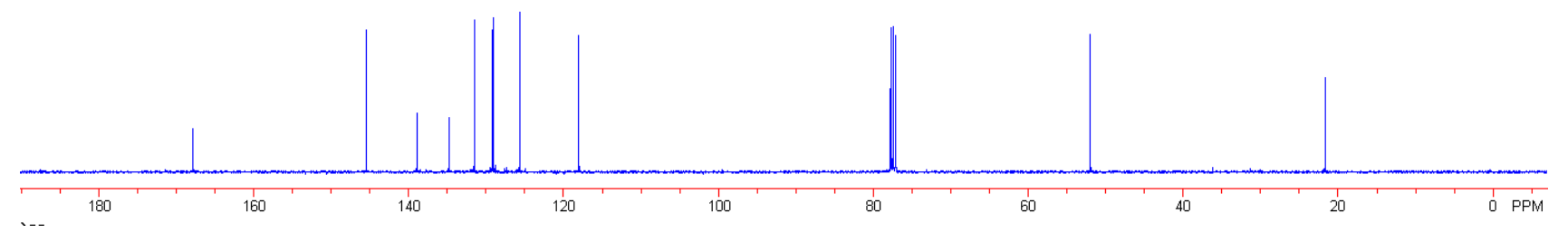



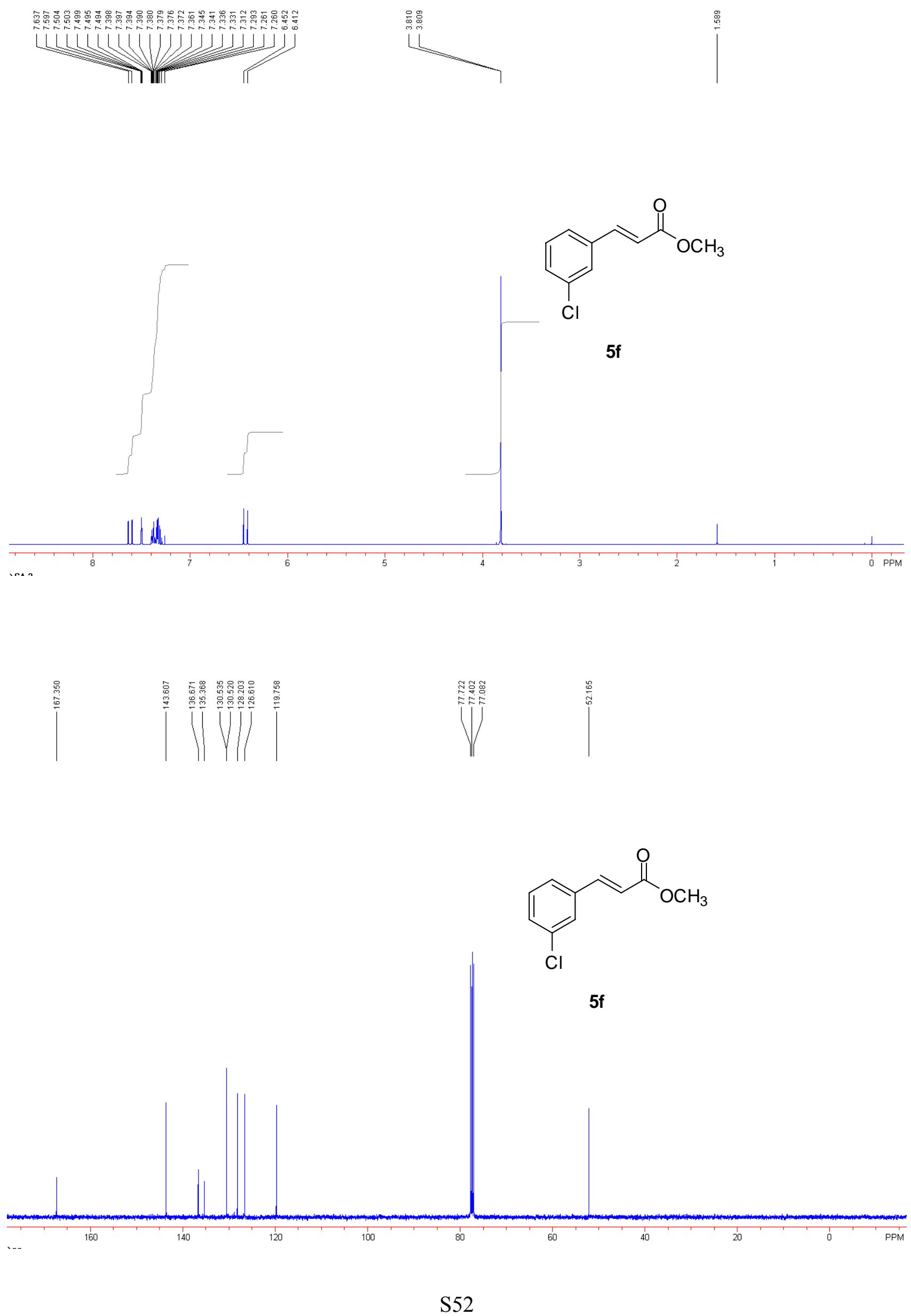

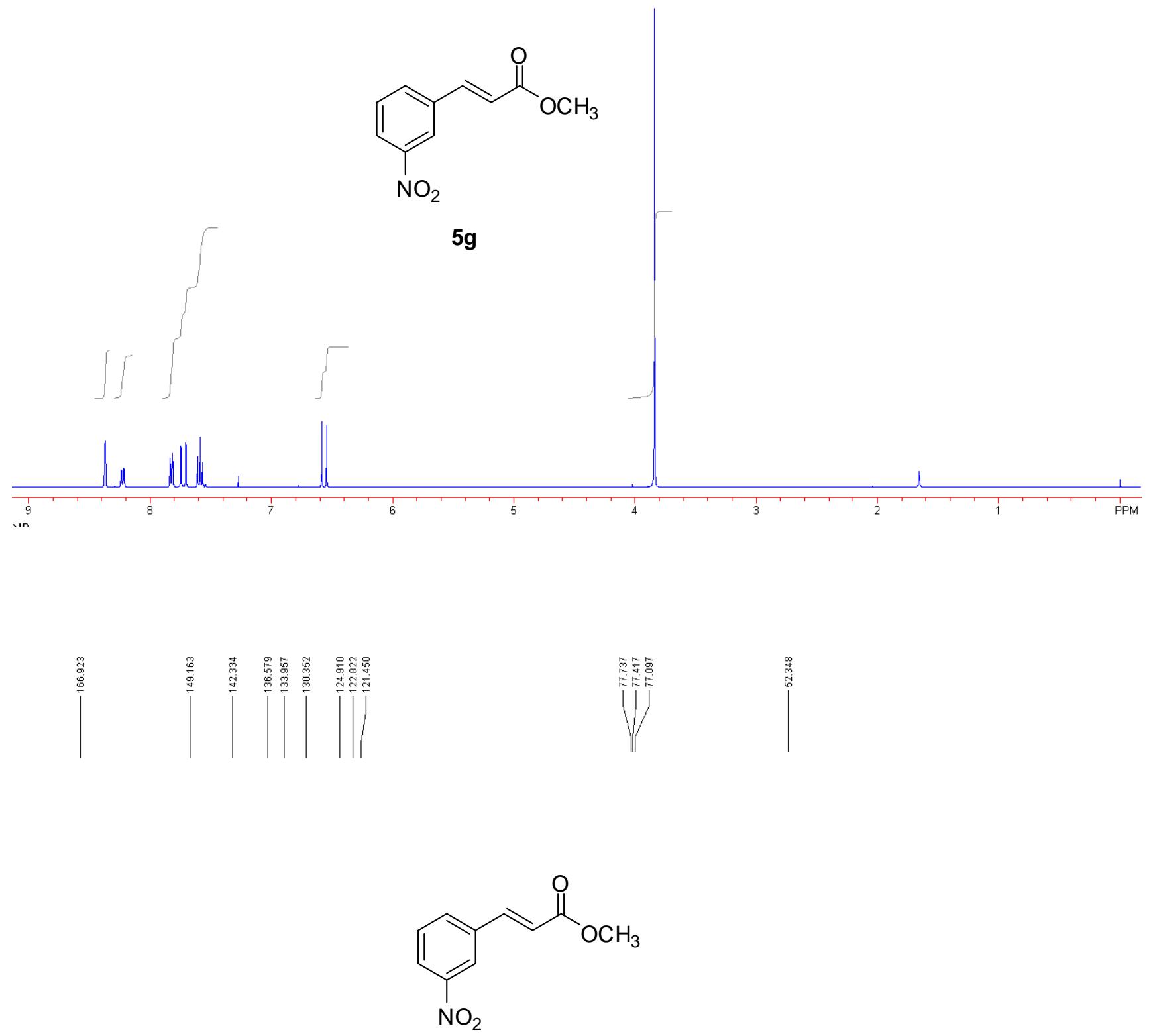

$5 g$
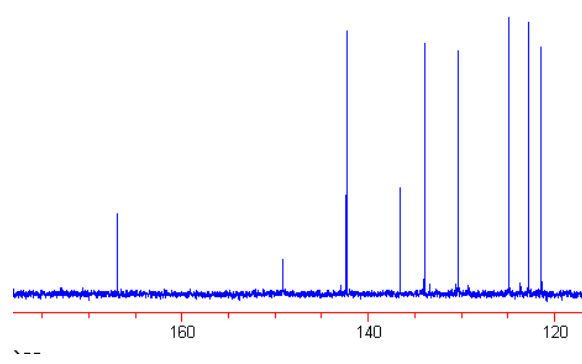
$\prod^{1}$
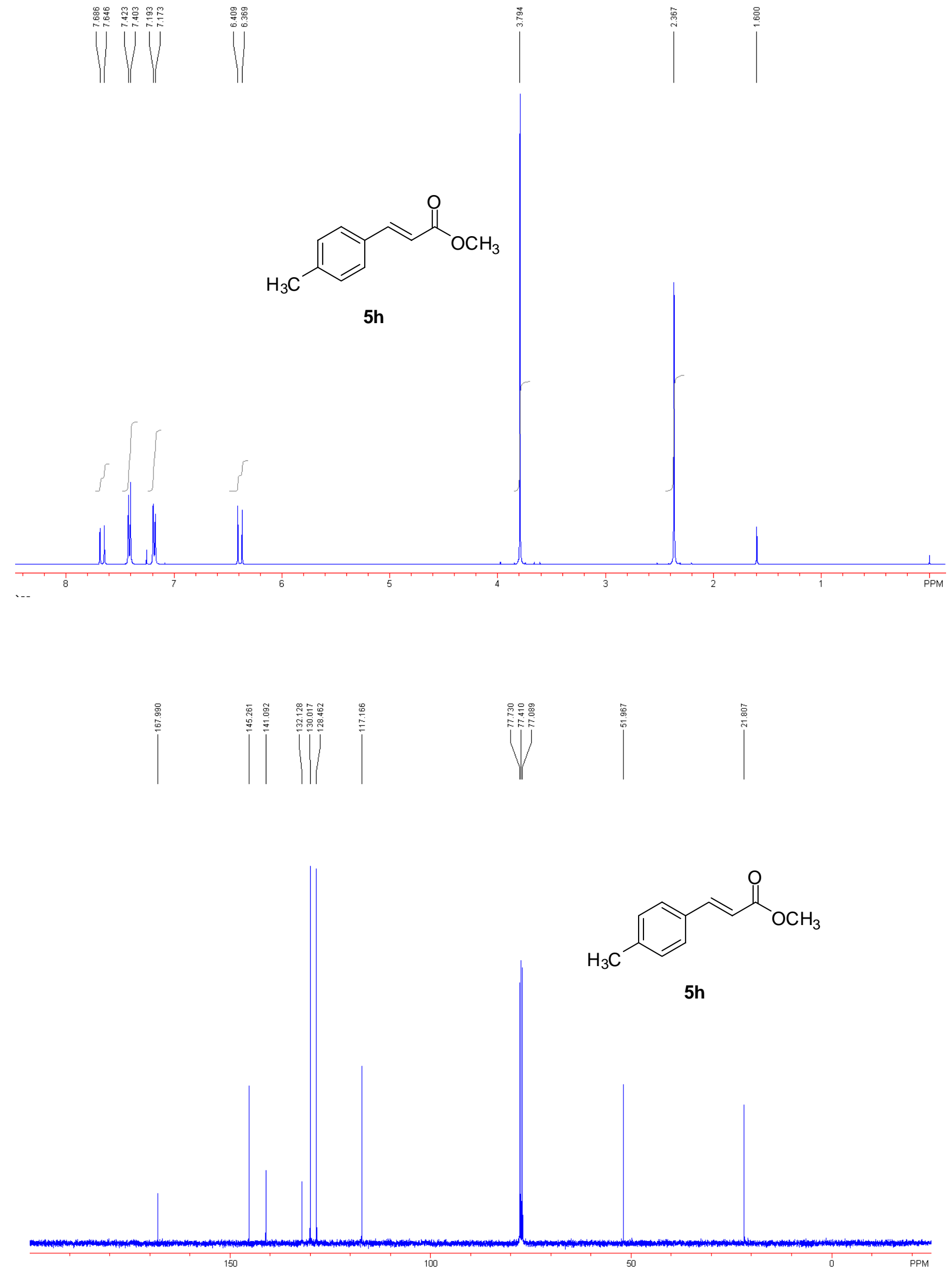


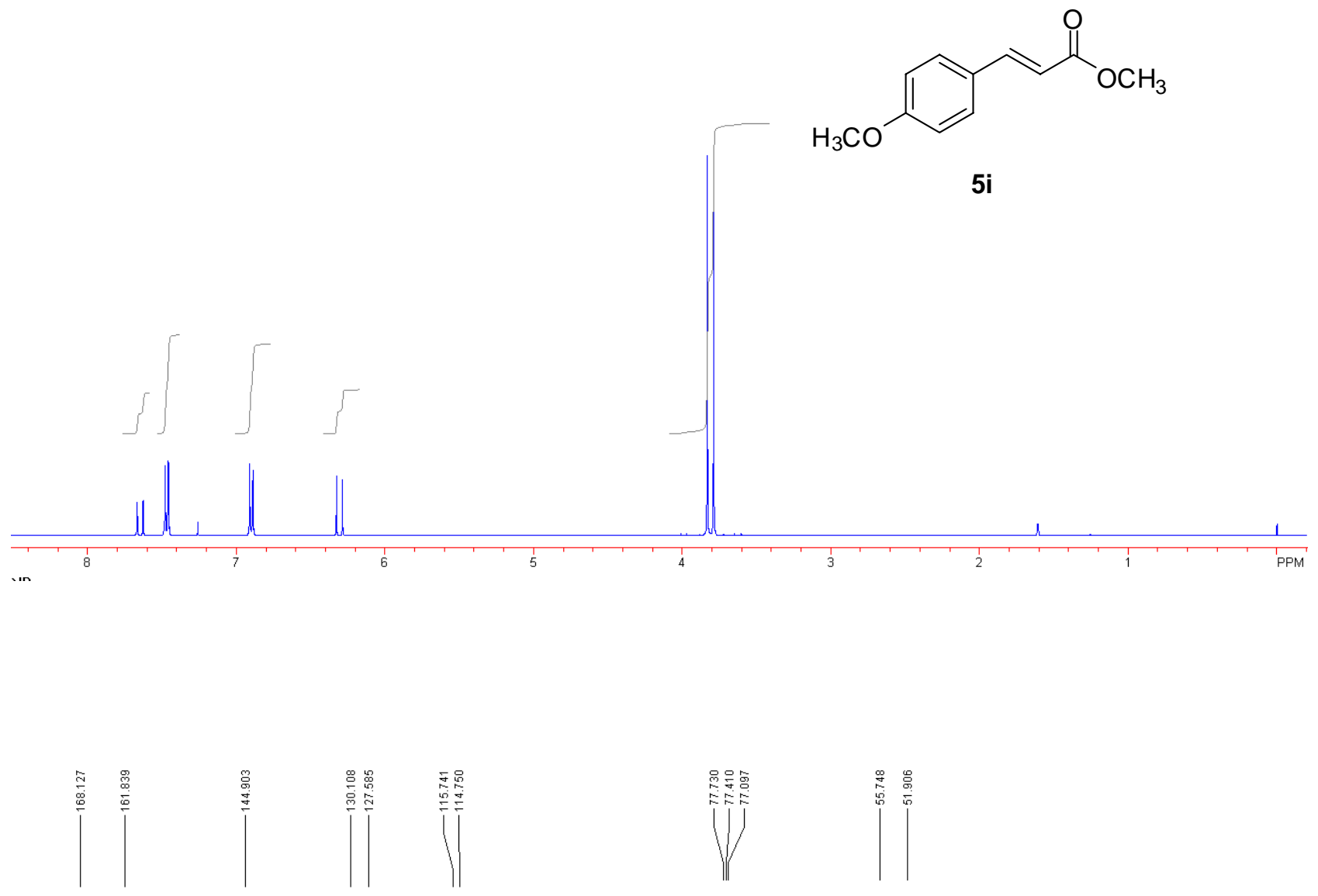<smiles>COC(=O)/C=C/c1ccc(OC)cc1</smiles>

$5 i$

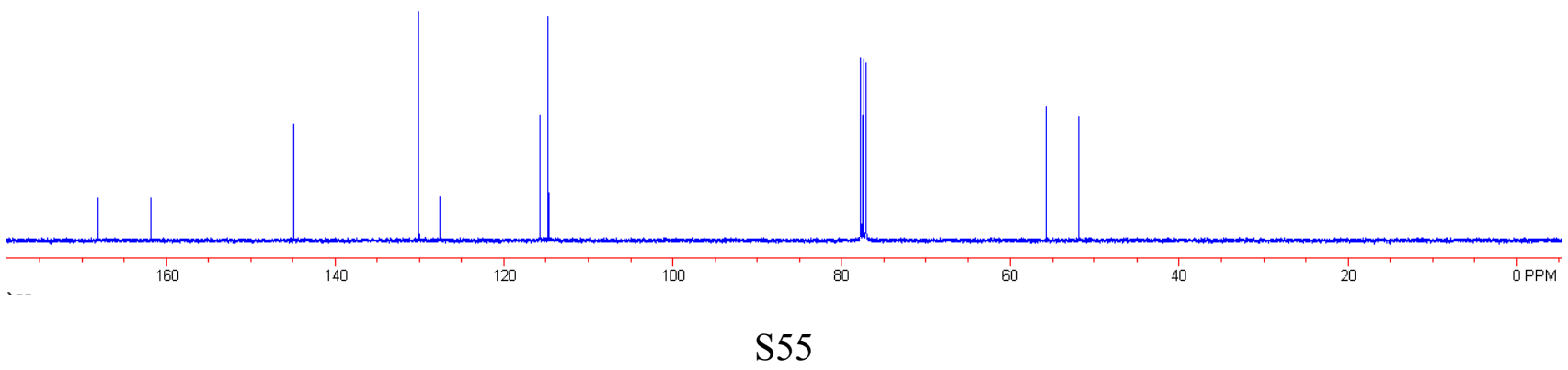




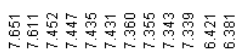

Uiviju
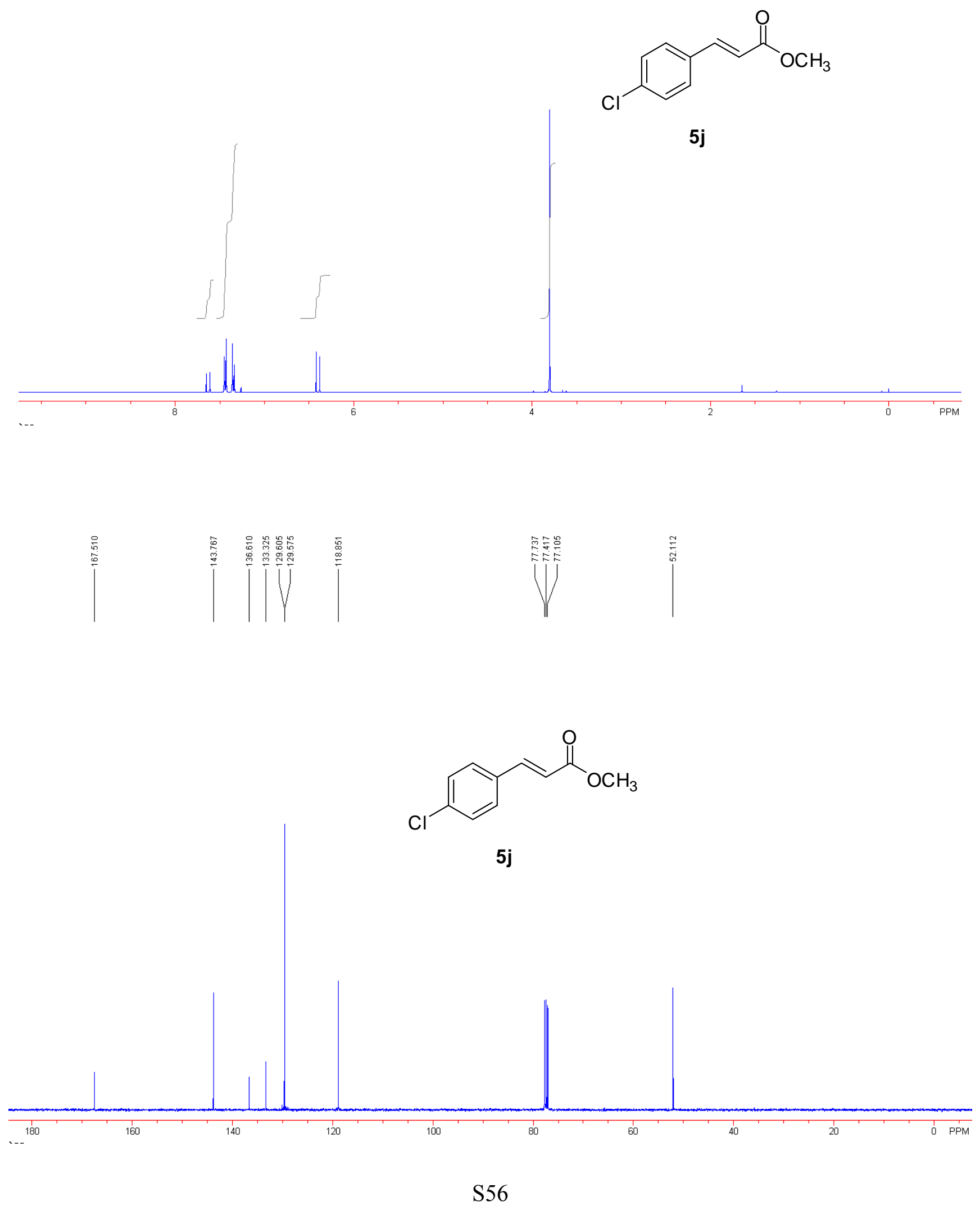


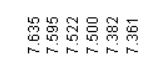

4\|\|$^{2}$
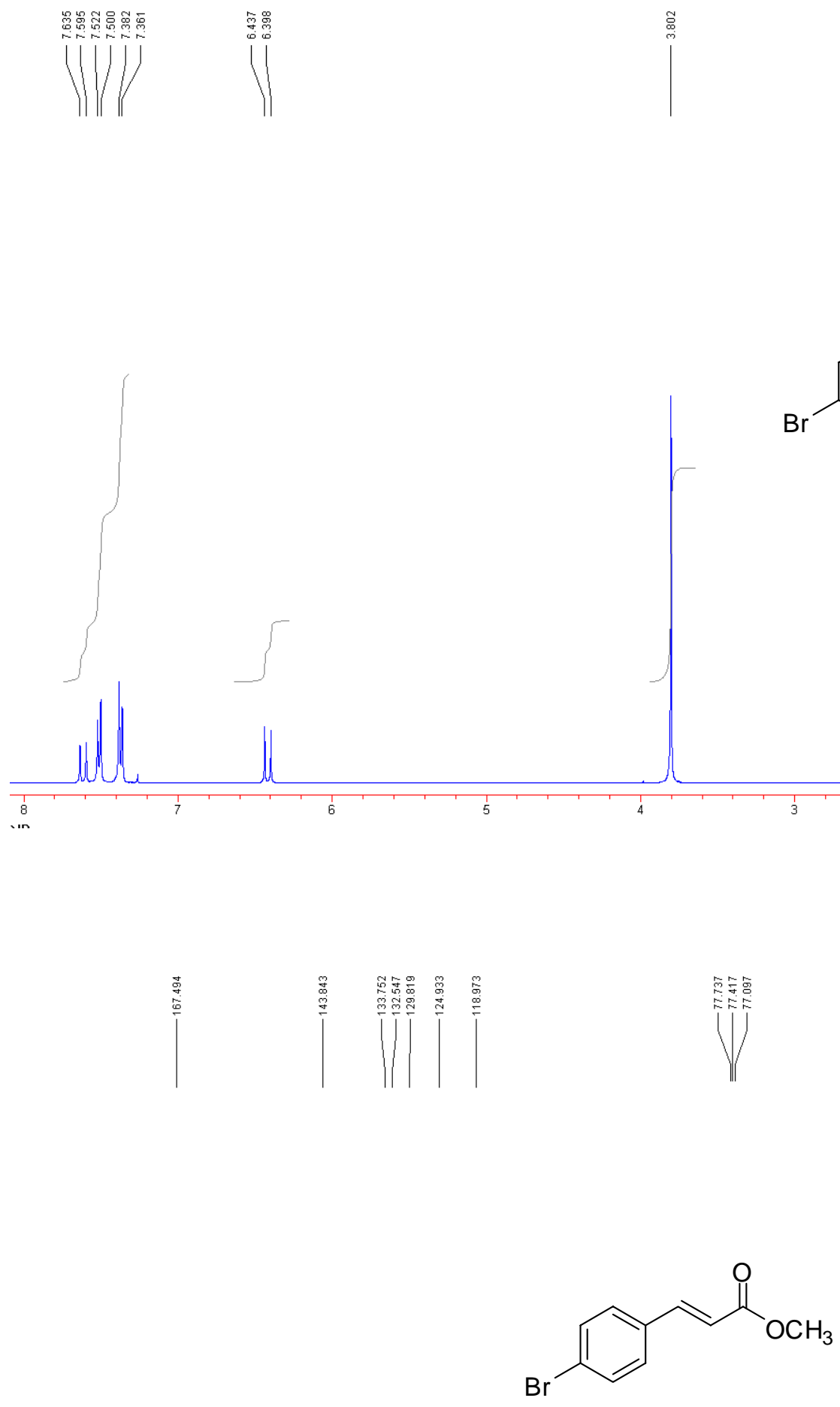

5k

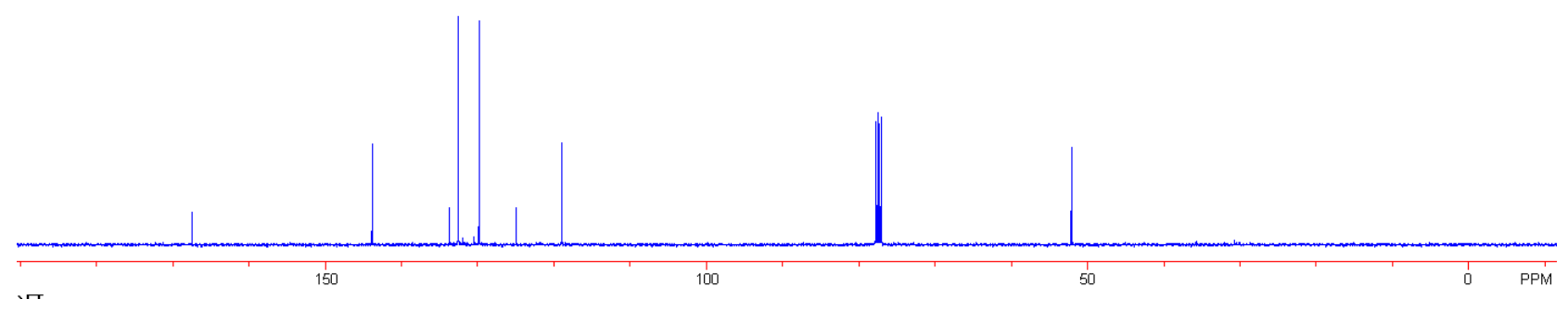



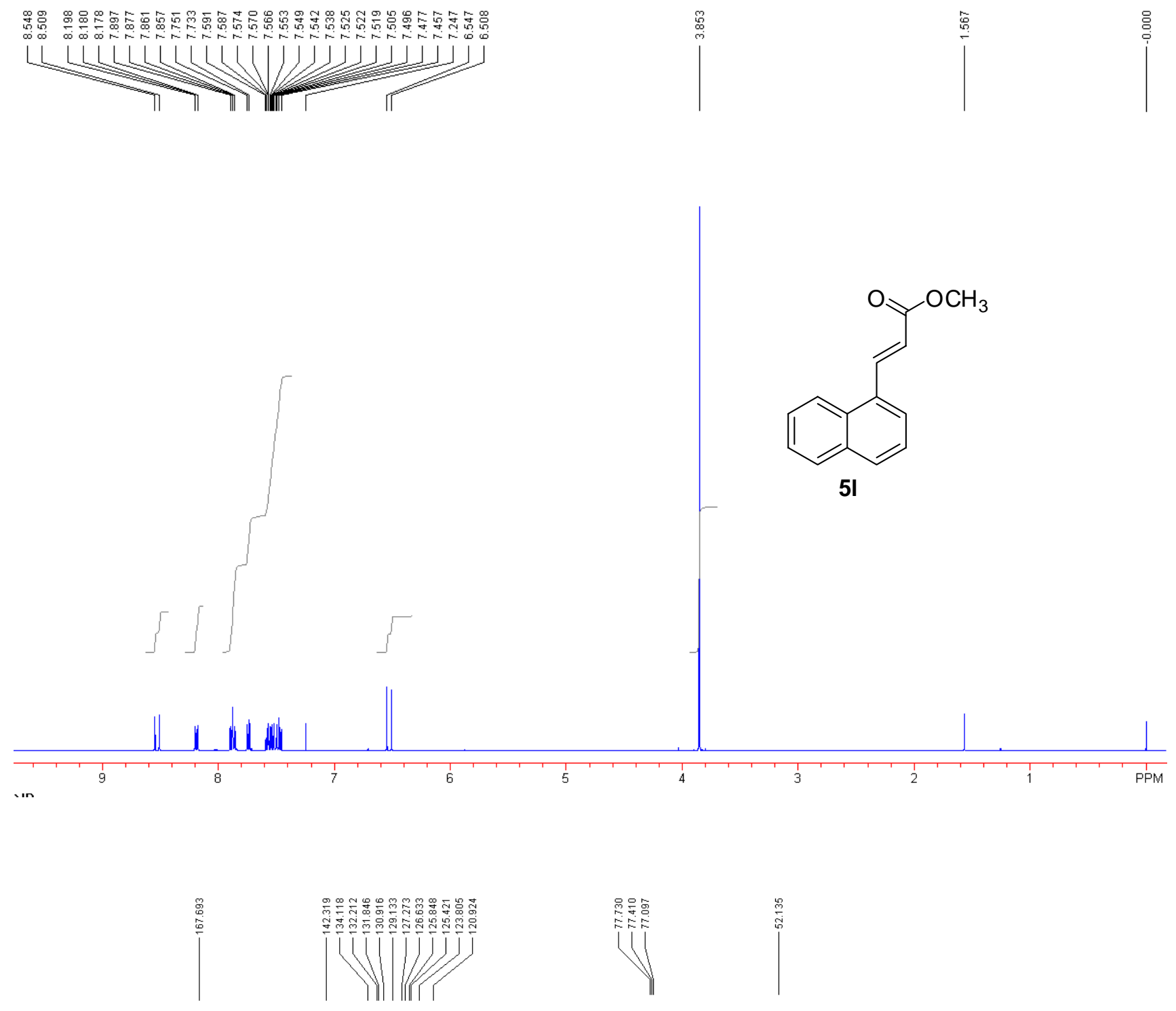<smiles>COC(=O)/C=C/c1cccc2ccccc12</smiles>

5

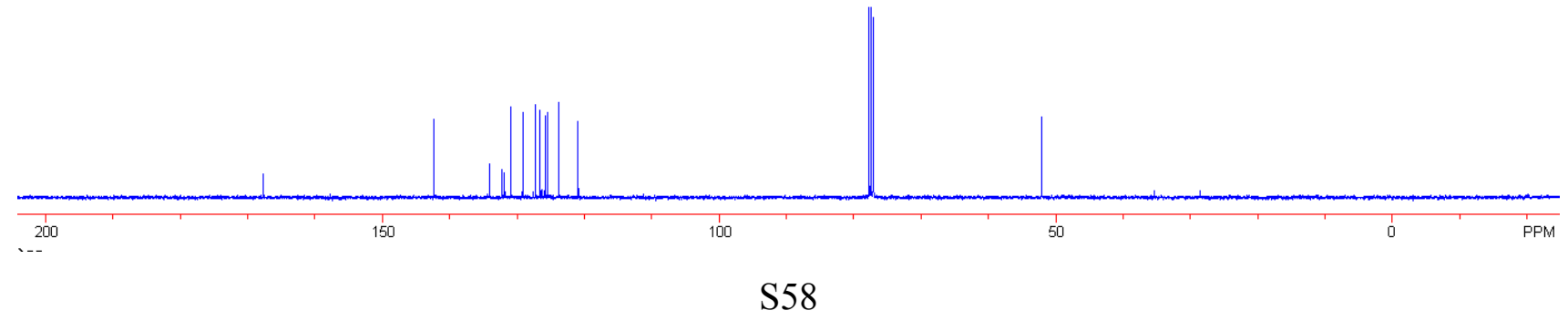



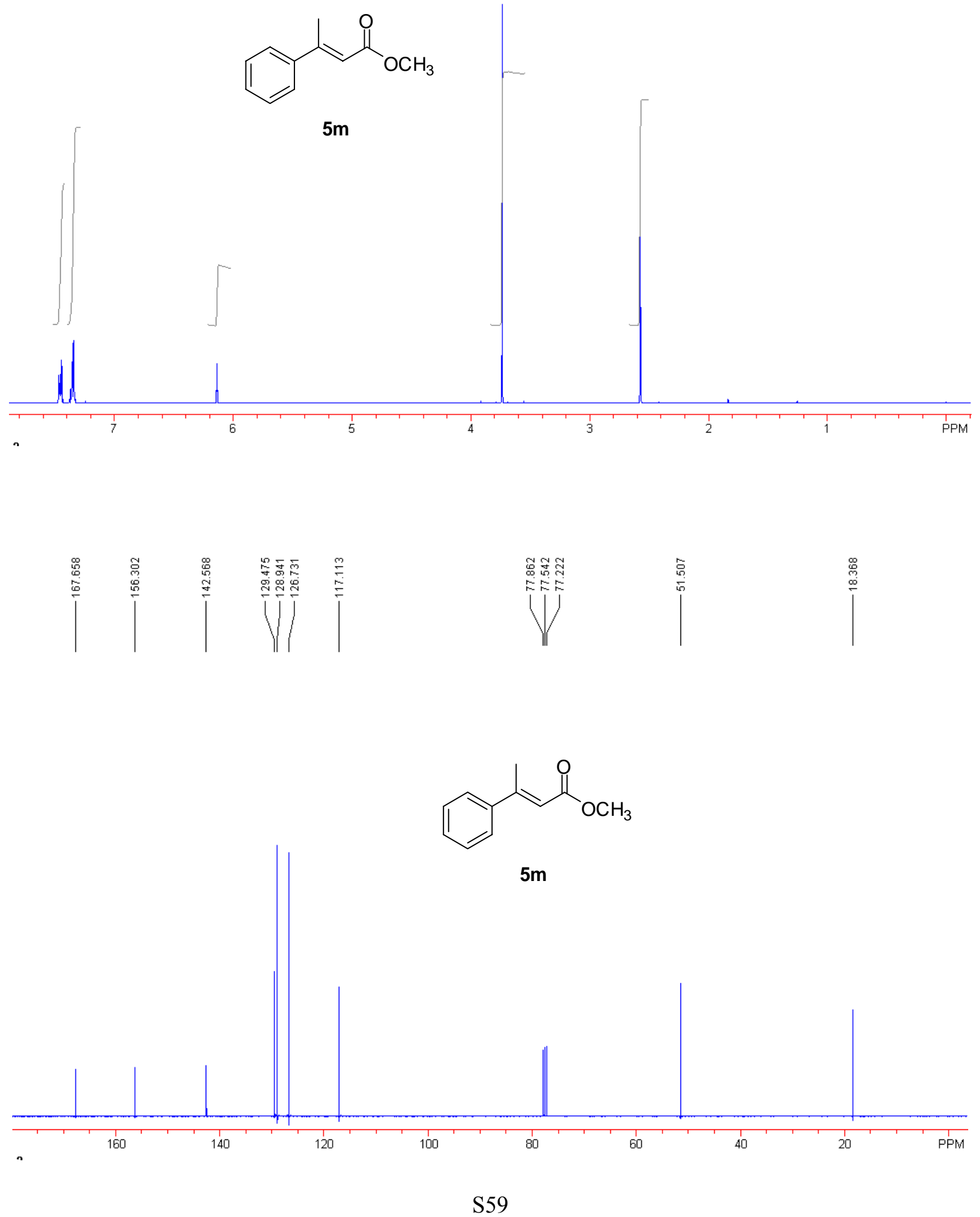

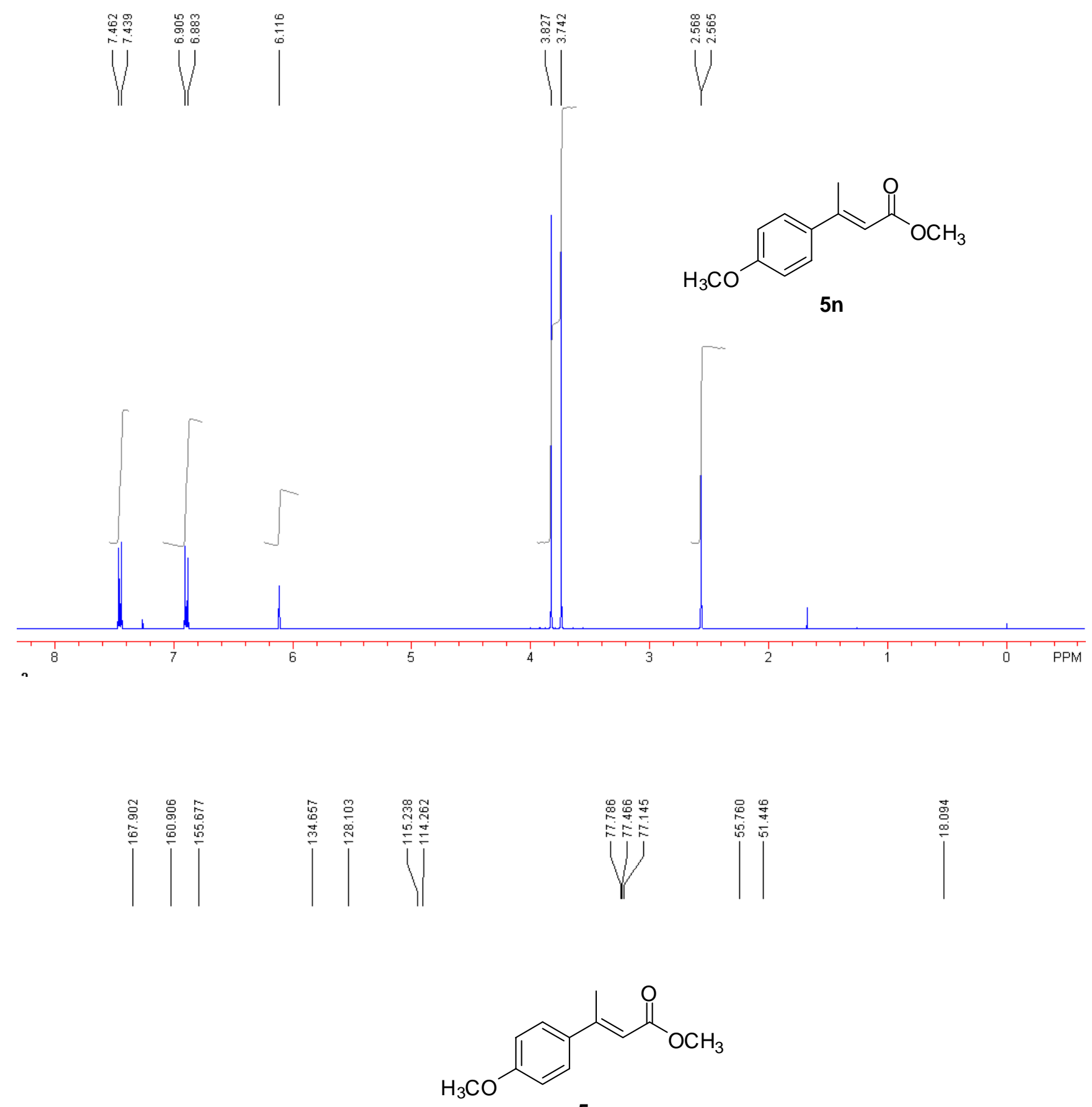

$5 n$

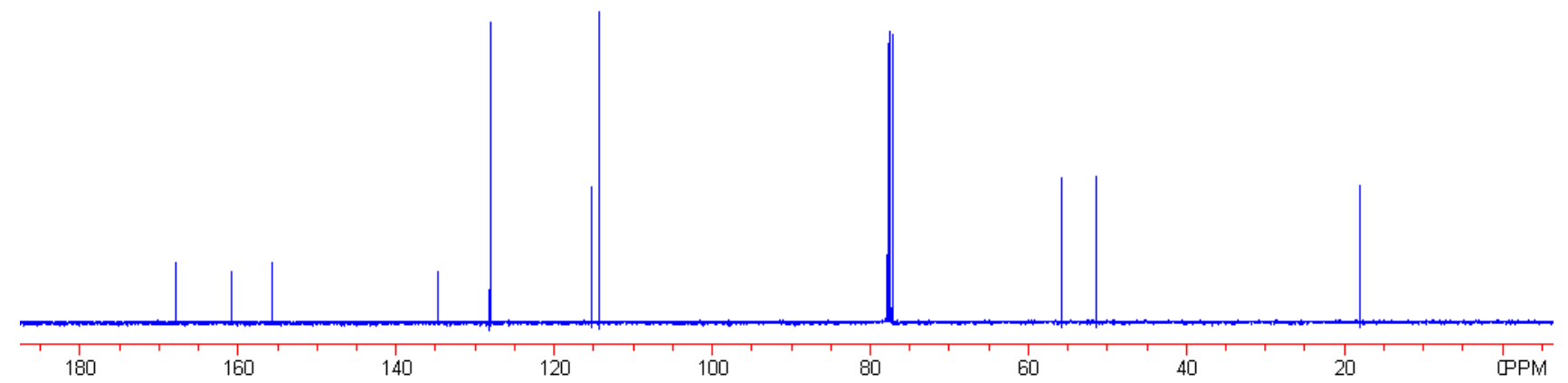


$\sqrt{|1|}$
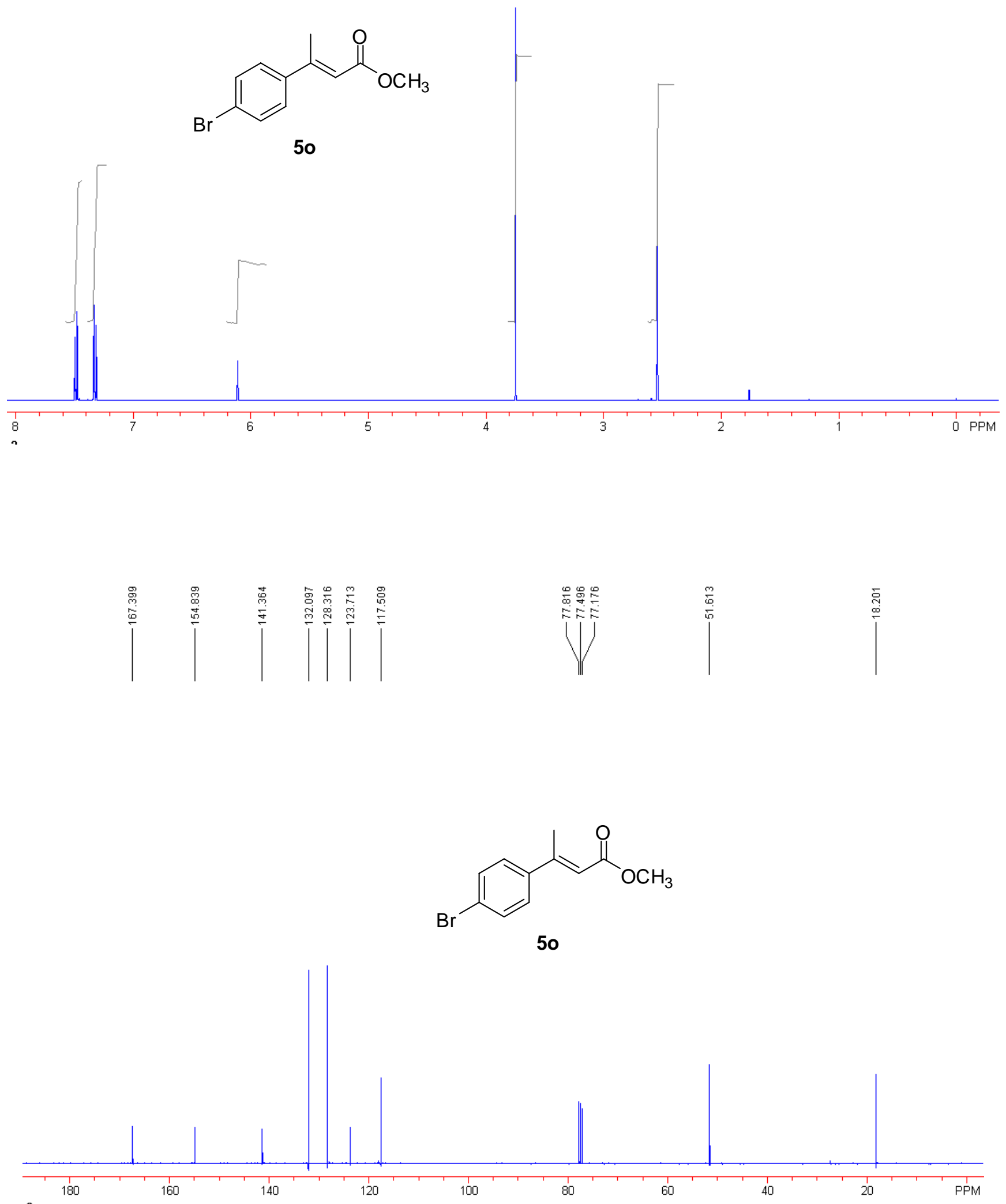


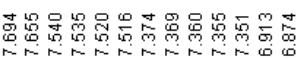

uMv
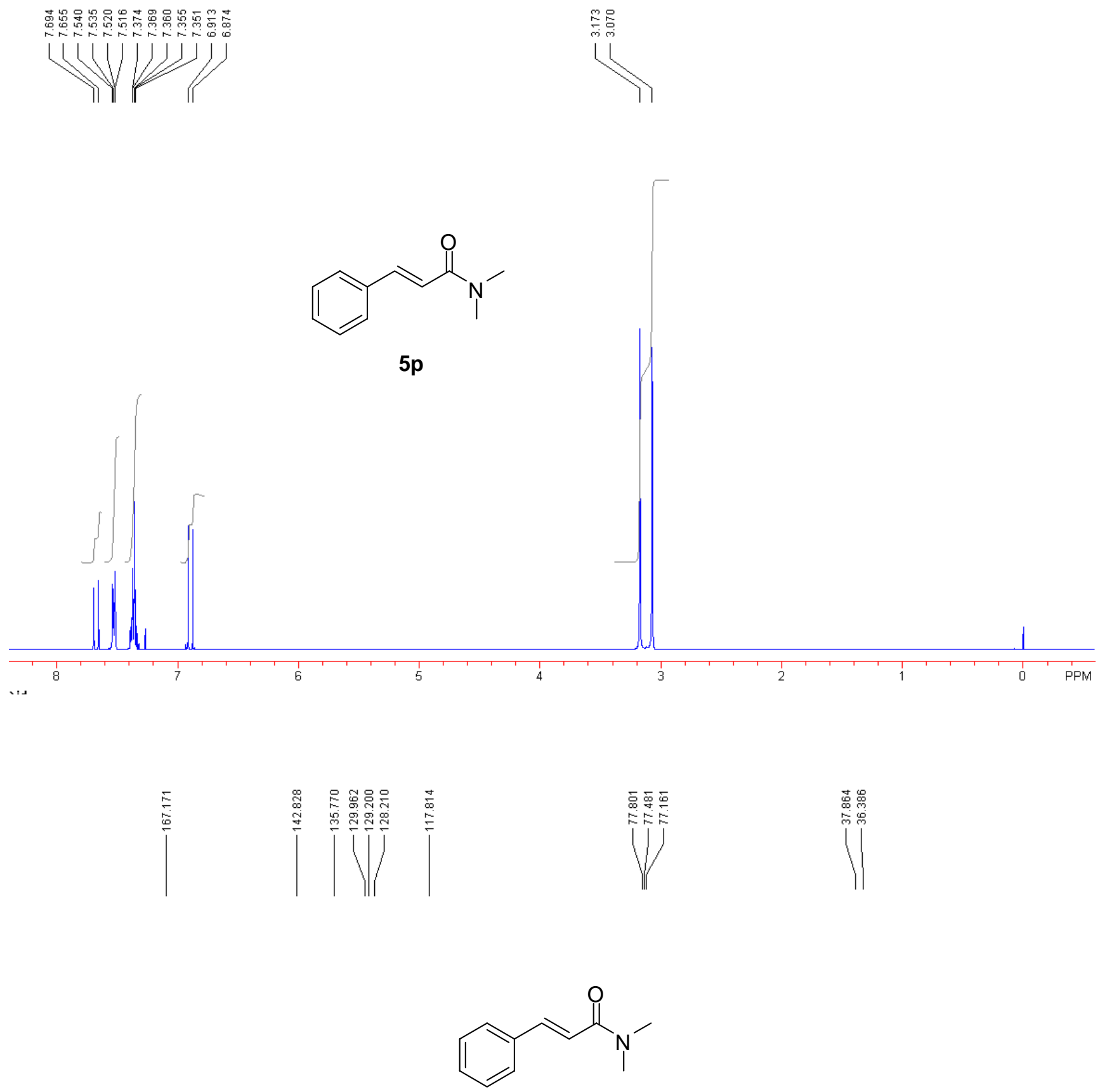

$5 p$

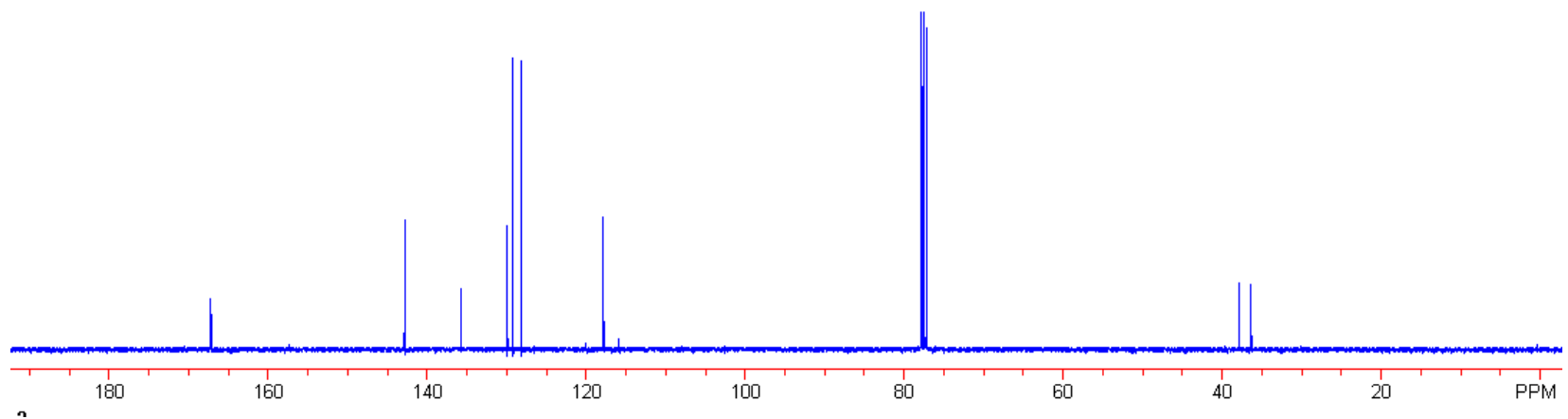




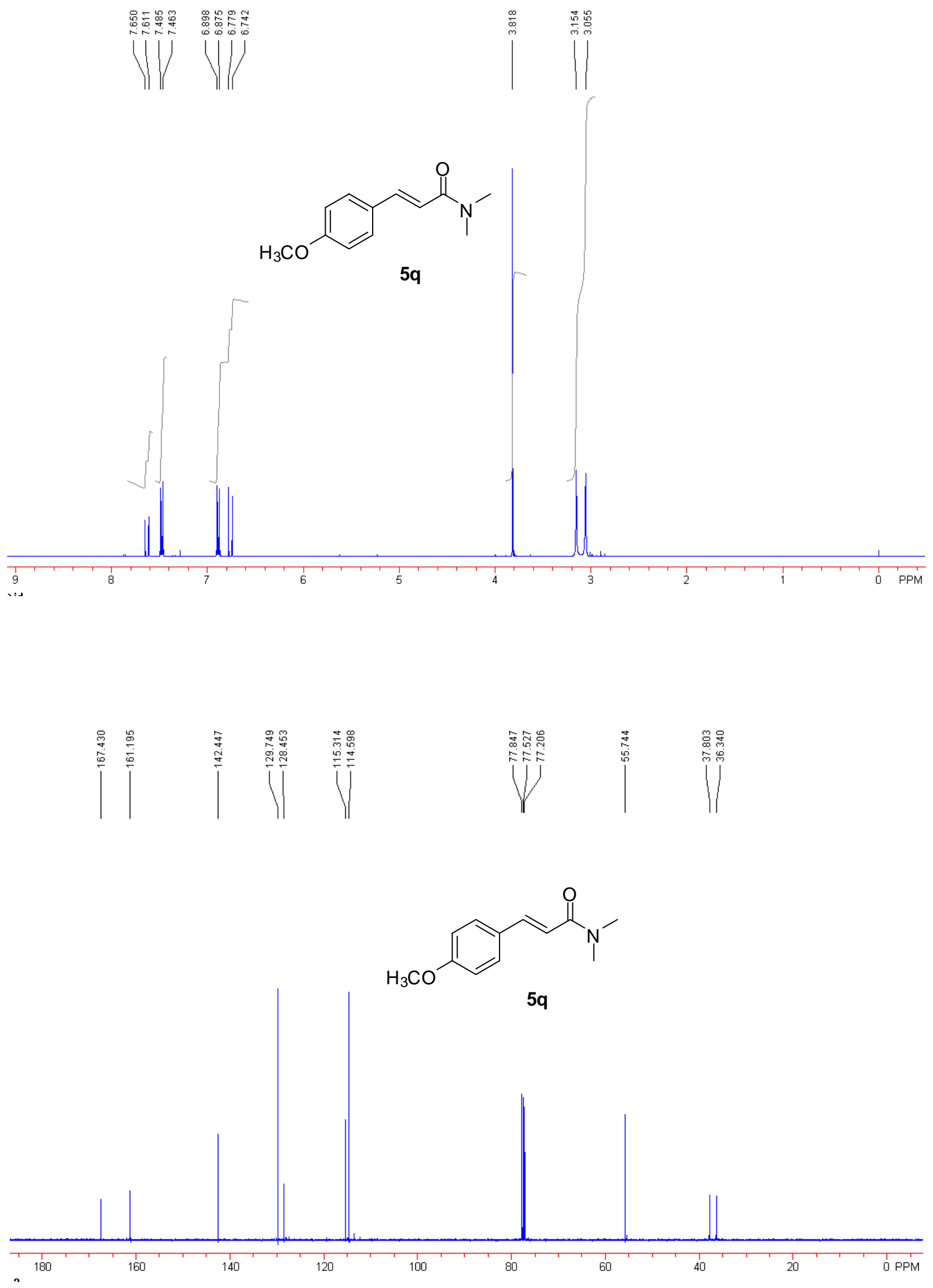




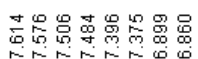

HII

吅哭
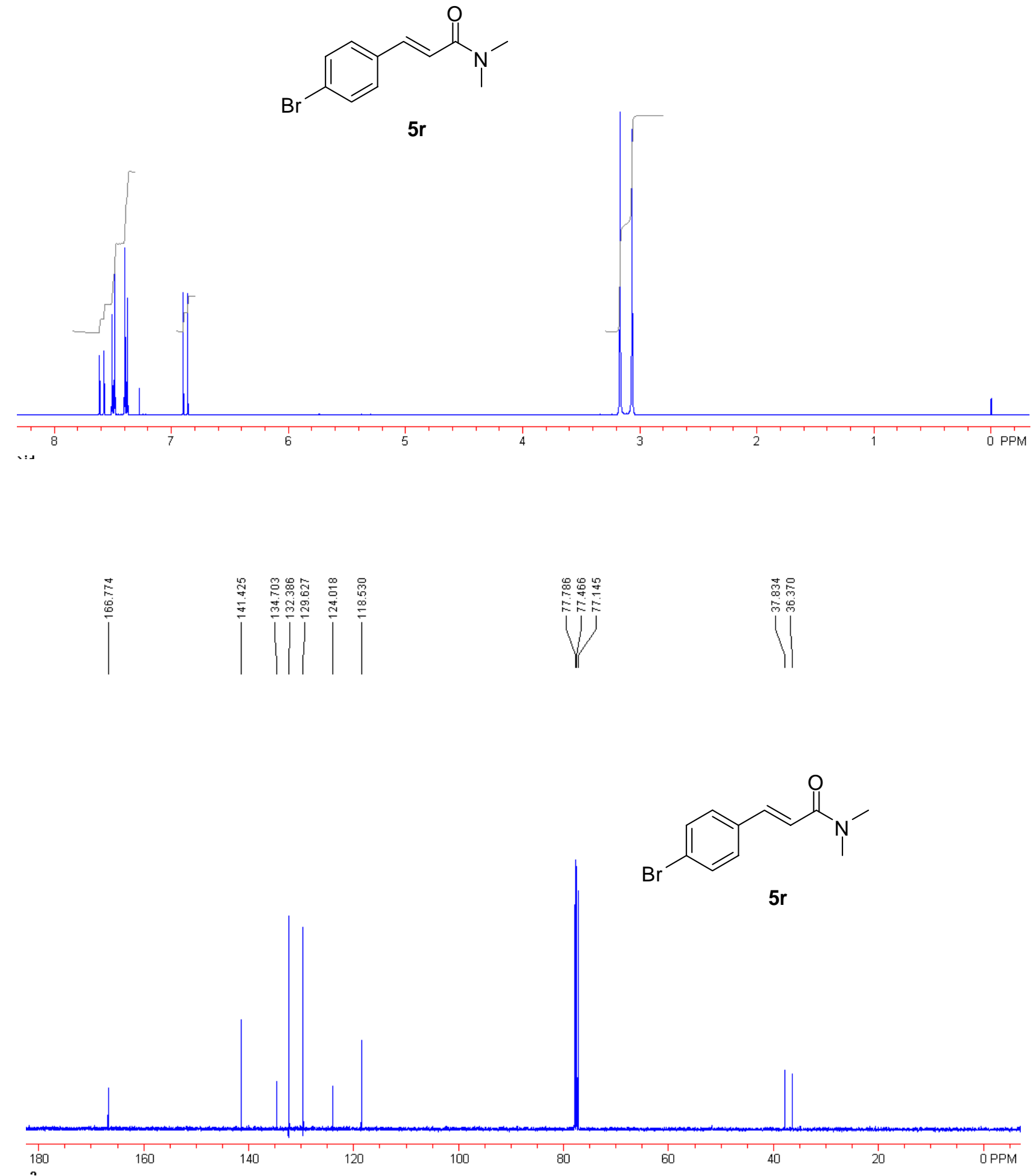

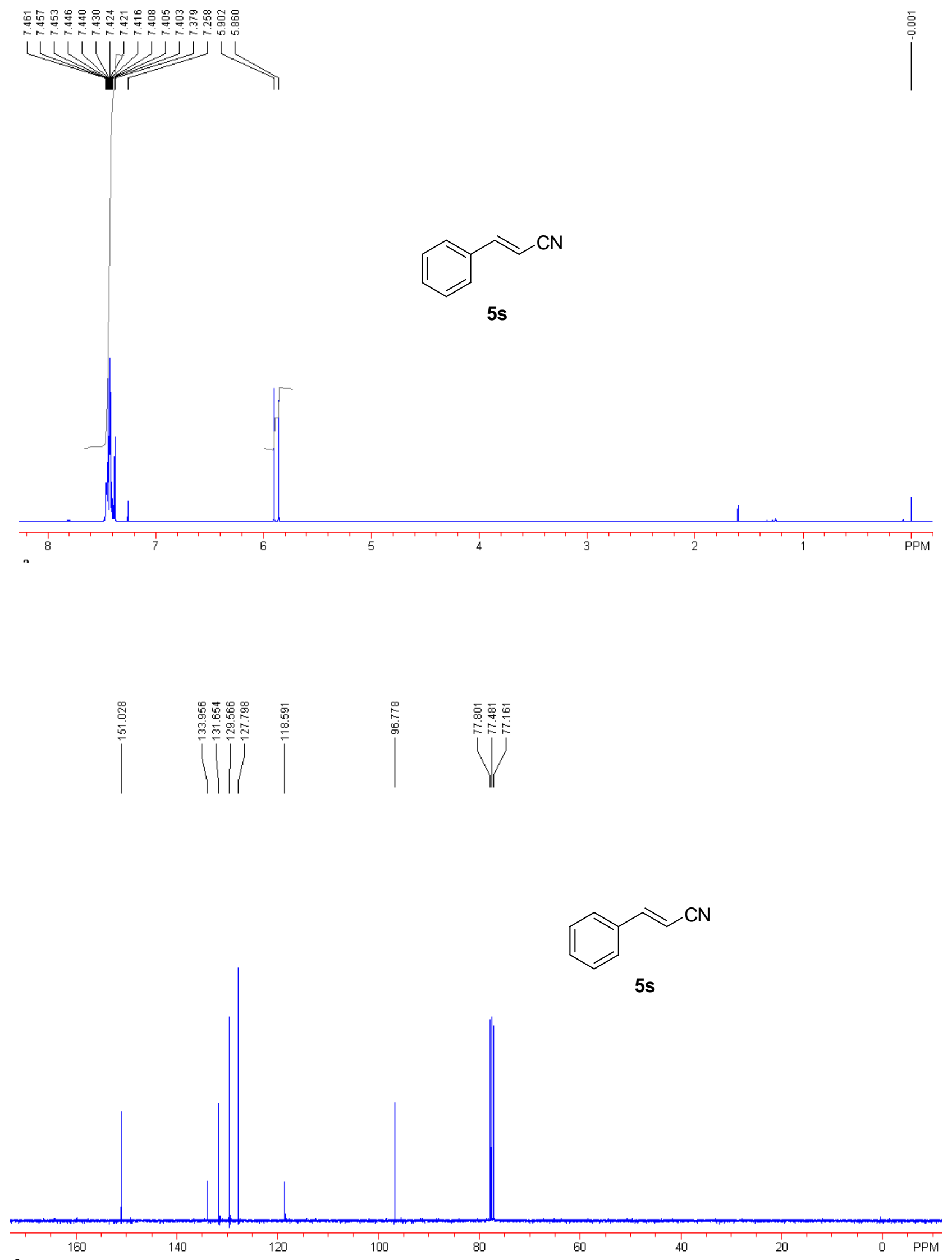


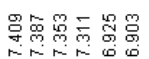

1)
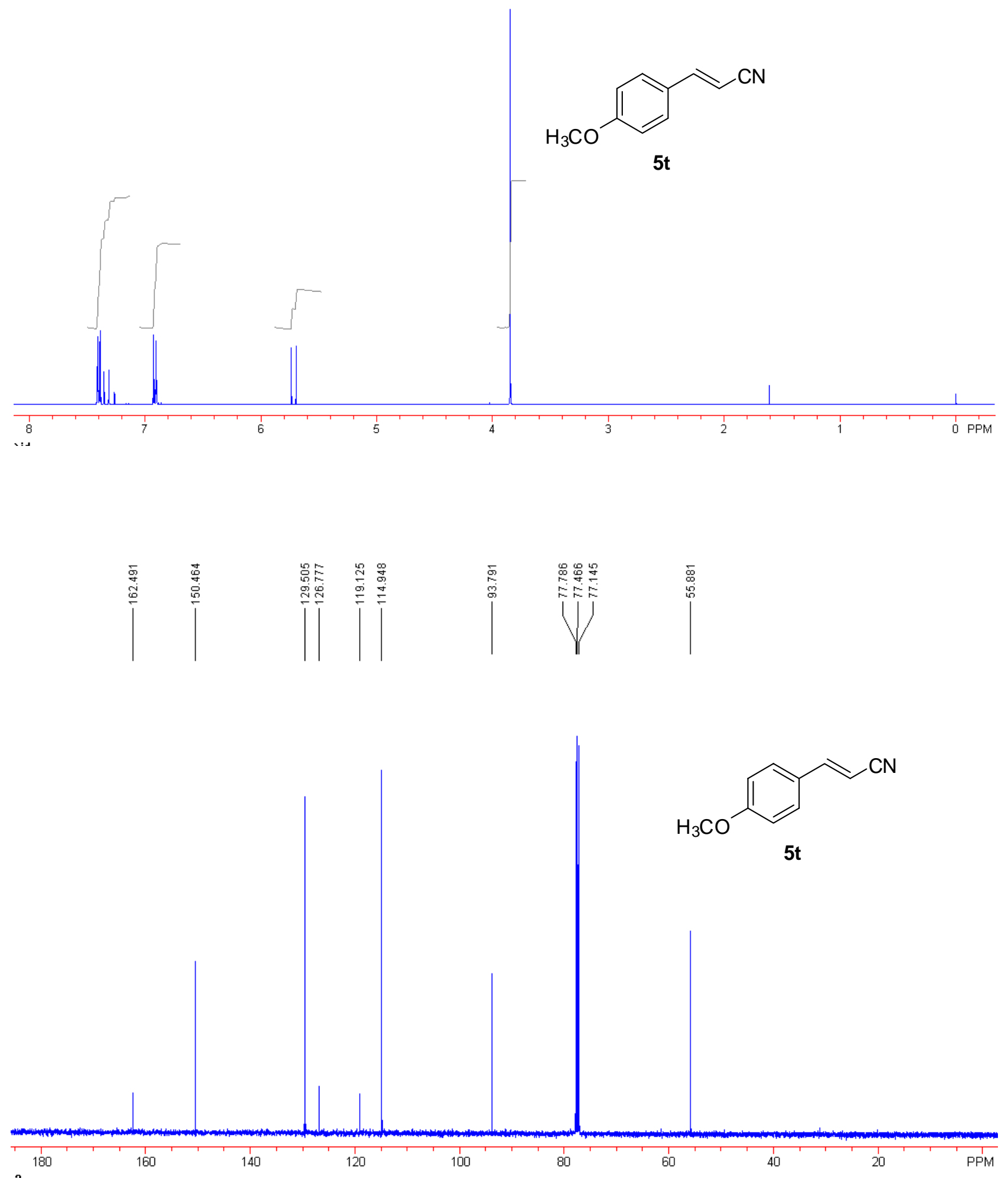


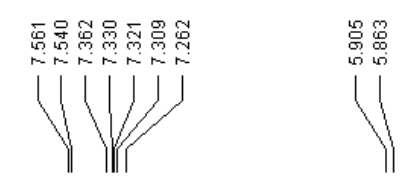
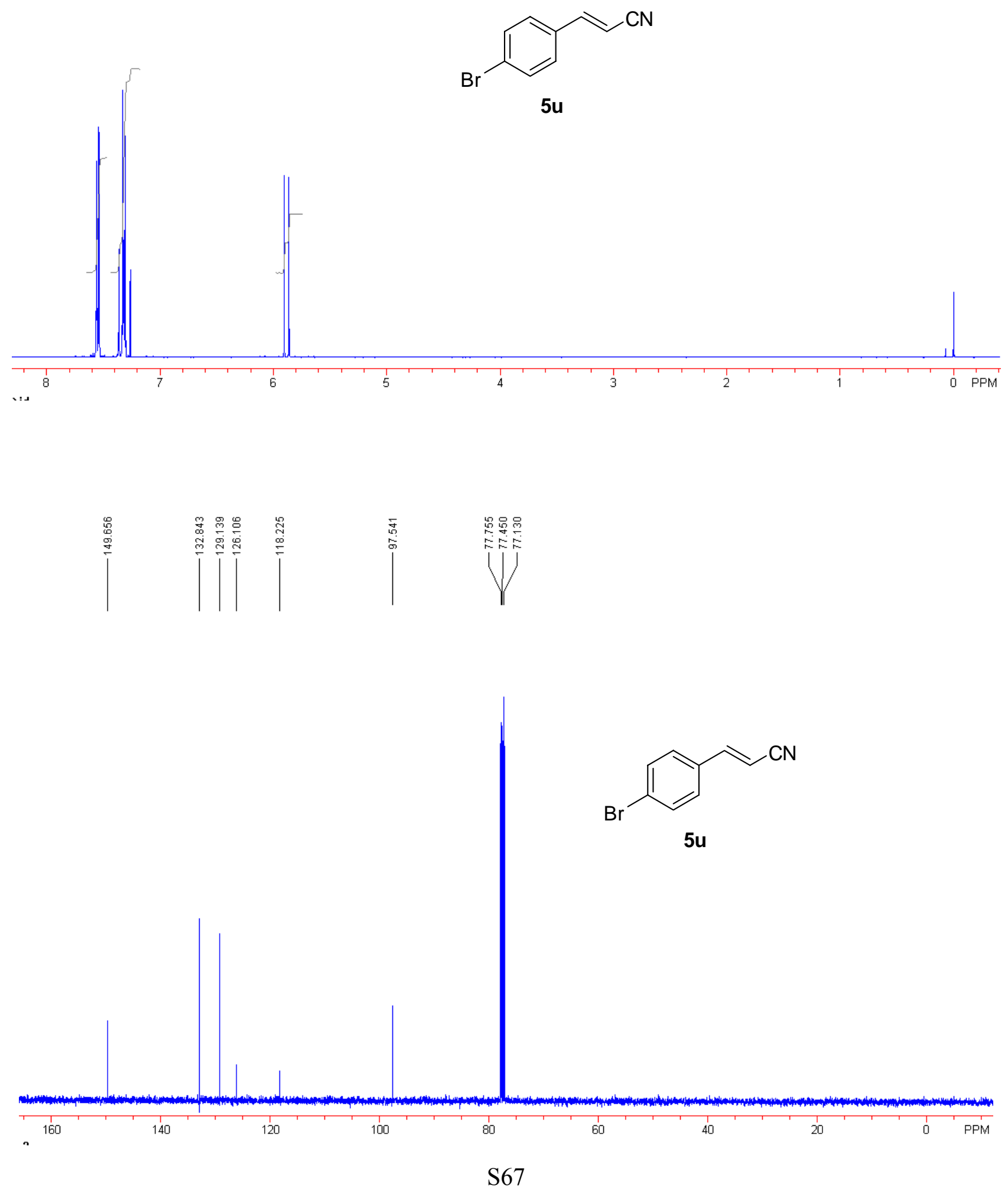